Article

\title{
Applications of Non-Standard Analysis in Topoi to Mathematical Neuroscience and Artificial Intelligence: I. Mathematical Neuroscience
}

\author{
Carmen-Elena Mocanu ${ }^{1}$, Florin F. Nichita ${ }^{2}$, and Ovidiu Păsărescu ${ }^{2 *}$ \\ ${ }^{1}$ Carol Davila University of Medicine and Pharmacy, Faculty of General Medicine \\ 8, Eroii Sanitari Avenue, District 5, Bucharest, Romania; Carmen.Mocanu@ @stud.umfcd.ro \\ ${ }^{2}$ Simion Stoilow Institute of Mathematics of the Romanian Academy \\ 21, Calea Grivitei Street, 010702 Bucharest, Romania; Florin.Nichita@imar.ro \\ * Author to whom correspondence should be addressed; E-Mail: Ovidiu.Pasarescu@imar.ro; \\ Tel: (+40) (0) 2131965 06; Fax: (+40) (0) 213196505
}

\begin{abstract}
This work - in two parts - tries to promote a new method in Mathematical Modeling, not ever used before, namely the use of the methods of Non-standard Analysis in Topoi. For the general mathematical theory of topoi (Topoi Theory) we refer to the book [10] . For non-standard analysis in the particular Boolean topos SET (the category of sets) we refer to the book [11]. This first part of the work is related, from an applicative point of view, mainly with Mathematical Neuroscience, more precisely a model of the human thinking, consciousness and subconsciousness. The connections between brain and mind will be also scketched, but a closer study also need some further topics to be studied in Part II: Artificial Intelligence, namely (Quantum) neural networks and (Quantum) Turing machine. However both parts (I. and II.) should be considered together, because they complement each other. The topoi model the intuitionistic logic (multi-valued) and have been used in Quantum Physics (see [9] and its references) while Non-Standard Analysis in SET (introduced by Abraham Robinson[31]) has been applied in Mathematical Economics (see [2] and its references); however, the combination was never used until now in Applied Mathematics. Even more, the Non-standard Analysis in Topoi is not too much studied from the point of view of Pure Mathematics either. One of the main objective of this work is to produce progress in this aria of Mathematics also, mainly for the SET-type topoi and exponential topoi, those topoi used in Quantum Physics and in which we need Non-stantard Analysis for our purposes. Based on the paper [13] we propose the logic of non-standard
\end{abstract}


extensions in topoi as a model of the human thinking (based on infons), these theories representing top and very difficult results in Abstract Mathematics. We will analise the relation between brain and mind via the genetic-epigenetic interplay. More details will be provided in the Introduction, followed by a short presentation of the general theory, later connected with the (Quantum) Yang-Baxter equations in topoi. The connections between these equations and braid groups and knots, with intended applications in Neuroscience and Artificial Intelligence will be further analysed in Part II. Besides promoting a new idea of modeling, the present paper aims to promote the building of a Research Proposal for the European "Human Brain Project" (https://www.humanbrainproject.eu/en/), and the NSF-Europe Program "Collaborative Research in Computational Neuroscience" (https://www.nsf.gov/funding/pgm_summ.jsp?pims_id=5147), jointly with the new founded "National Centre for the Research of the Brain" of the Romanian Academy of Science (https://acad.ro/centreAR/CNCC/CNCC.pdf, in Romanian). "Blue Brain Project" (https://www.epfl.ch/research/domains/bluebrain/) could also be of big interest. We hope that the journals edited by MDPI (Axioms, in particular) and by the Simion Stoilow Institute of the Romanian Academy - Revue Roumaine de Mathematique Pures et Appliqués (http://imar.ro/journals/Revue_Mathematique/home_page.html) - among others - will host special issues related to the research subjects described in both parts of this work, starting with this one. For further details and some of the progress on this research subject, see www.ovidiufpasarescu.com.

Keywords: non-standard analysis, topos theory, Yang-Baxter equation, universal gate, quantum computer, brain studies

Classification: MSC 18D35, 16B50, 16T25, 18F10, 03B50, 26E35

\section{Introduction}

This first part of our paper is related to the Neuroscience, viewed from a mathematical perspective. Nevertheless, both parts should be considered together, because some of the notions used in this first part will be detalied in the second one, so the notions equally apply to both topics. This paper addresses to an auditorium of a great diversity: neuroscientists, informaticians, physicians, physicists, biologists, mathematicians,... aiming to produce an interdisciplinary group of research. With these things in our mind, we tried to write a paper which could be read by different kind of specialists. Even the authors of this work in two parts and their collaborators belong to various scientific areas (neuroscience, mathematics, medicine, economics; professors, researchers, students), with the hope that we produced an enough self-contained and readable paper by many categories interrested in the topics. Although, due to the previous reasons the paper shortly re-explain already known notions and results, it also contains new and original contributions. The main original contribution of this paper is the idea of considering the non-standard extension of the intuitionistic logic (in topoi) as modeling the human 
thinking, consciousness, and considering together both natural and artificial intelligence, as part of the global brain, as explained in section 3. We also contribute to the development of the Non-stardad Analysis in set-type exponential topoi, in the aria of Pure Mathematics. Information and energy represent the two facets of an elementary particle in String Theory (the string vibrates = energy in a specific way (amplitude, frequence, $\operatorname{spin}, . .$. ) = information, in order to give one or another one of the elementary particles). They do not reduce to our brain, neither to our body, they travel freely realising connections between the interior (brain, senzory organs, DNA = genetics) and the exterior (epigenetics) of the human body, generating the mind, reciprocically related to the brain (see [29], [7]). We deal with these subjects in more detail in sections 2.,3.,and 4. In sections 5.- 7. we recall the basic notions of our modeling approach (based on Topos Theory and its Non-standard extension), the examples from section 7. being original (non-standard analysis in Set-type topoi). The interpretation of theories (from mathematical logic, model theory) and of the corresponding categories (models of the theories) as infons, energons, and receptons represents an original approach also. For details concerning these things, see sections 4. and 8. Finally, the use of the theory of knots and braid groups - and, as a consequence the Yang Baxter equations - in neuroscience, represents a new idea also. One of the most facinating theories of the last centuries is the knot theory. Some of the most brilliant minds struggled with the task of classifying knots. They found polinomials which distinguish knots (and for that purpose the Yang-Baxter equation was employed). In section 9. we recall the Yang-Baxter equations and produce logical solutions in topoi, finding in such a way connections between Topoi Theory (so, intutionistic logic) and the Yang-Baxter various equations. We intended to make this paper as self-contained as possible, in order to make it readable, being addressed to a variety of specialists, not having - usually - the same academic formation. Hawever, there are some notions used in this first part, but not explicitely recalled'as there are: (Quantum)Neural Networks, (Quantum)Turing machines, and further developments of the Non-standard Analysis in more general topoi. All these things will be considered in the second part of this paper (II. Artificial Intelligence), together with more details concerning the connections between knots, braid groups, Yang-Baxter equations, and DNA, brain, quantum computers.

\section{The Role of Information in the Structure of Matter}

First of all, information is related to organisation. We say that some systems or objects are more complex than others because we perceive them as containing more information. For example, it is obvious that an epithelial tissue contains more information than a cell. Epithelial cells exhibit distinct polarity, having an apical, a lateral and a basal domain. This organisation involves more information, as the molecular mechanism responsible for this arrangement is required to create a functional barrier between adjacent cells. Following Tom Stonier's idea from [30], information is related to entropy, because the latter is a measurement of organisation. They are exponentially inverse proportional: as a consequence, if - inside a complex system - the entropy decreases, meaning that matter is more organised, the amount of incorporated information increases. We think it is really important to see how this new concept - the incorporated information - influences our lives and more specifically the role it plays in the structure of matter. How do atoms know how to bind together and form a more complex structure? How can a simple stem cell differentiate in so many other types of cells? Information dictates 
all those processes. Everything contains infons (see section 8). Let's take as an example a sound wave. We can tell for sure that it contains some kind of information, that travels through air until it reaches the human ear. Here it is transformed into a nervous impulse and travels to the brain, where it will be perceived. A sound wave is a mechanical wave that causes local regions of compression and rarefaction through the medium, deviating the local pressure from the equilibrium pressure. Therefore, sound, which is made of information, changes the structure of matter. In neuroscience it is of extreme interest to see how information affects the brain. We, as humans, are born with unconnected neurons. In the first two years, we form synapses while experiencing the world around us. As we grow up, we lose almost $50 \%$ of the already formed synapses, but we also form others while interracting with the environment. How does information influence this process? The more we interact with something, the more powerful the synapse gets. It's like a road through a forest. At first, there's nothing there, but as more and more people go along it, the grass disappears and a path is formed. The same thing happens in the brain. We believe that information extends one's life. During the first part of our lives, the human organism functions in an anti-entropic way. As it gets more organised, the entropy decreases, instead of increasing. However, after this period, the entropy of the human organism continues to increase until it reaches a state of equilibrium with the environment, when the organism dies, because no more energy exchange can be done. By increasing the amount of information one takes in, the entropy of the organism decreases, meaning that it prolongs the process of ageing. Thus, learning new things delays the decay of the brain primarily, but of other organs as a consequence as well. For a much more physico-mathematical approach of such kind of things, see sections 4 . and 8 . of this paper.

Moreover, inside the human brain there are complicated structures formed by proteins, in particular very complicated knots, which contain information in their nodal structure. So, in order to understand how the information is encoded in the nodal structures of the proteins, we could use the mathematical connections between (Quantum)Yang-Baxter Equations(QYBE, YBE), Braid Groups, and Knots. Moreover, braid groups are also related to the study of DNA. In this paper, the first part of the work (section 9.) we recall the QYBE and YBE, and we find logical solutions of them in the classifying objects of a topos (with its Boolean or Heyting algebra structure, see section 5.and 6.). More details concerning the previous relations will be recalled in the second part (II. Artificial Intelligence).

\section{Life and Information: Brain and Mind versus Genetics and Epigenetics}

For further details related to this section we refer to the books [7], [29]. The Brain is the main organ where the mind is born, but the brain is far from being the only entity involved in the growth of the human mind. The mind is a by-product of the neural networks from the brain (synapses), in continous transformations (some synapses disappear while new ones are born), representing the so-called plasticity of the brain. The synapses are born as a consequence of the learning process - schools, various experiences - through the interactions between the human being and the environment. The mind is also a by-product of other kind of communications between neurons (and the glial environment), namely the quantum networks connected by the so-called microtubules. Certainly, this interactions are between the brain, through the sensory organs, moderated by the information from the DNA molecule (genetics), 
and the environment (epigenetics). The human being has much information in its DNA, but only some of it will be used in a life-long period. Simplifying, there is information that tells us to start trembling when it is cold (or to sweat when it is hot). However, if we spend a life-time period at the Ecuator we will never experience the trembling (respectively, if we spend a life-time period at the North pole we will never experience sweating). So, the information from the around $2 \mathrm{~m}$ long DNA molecule is read by the environment - epigenetics, the system genetics/epigenetics becoming a kind of (quantum) Turing machine, generating, in particular, the evolution of the mind, which, through plasticity, generates the evolution of the brain, and vice-versa.

We - the humans - think using both consciousness (awarness) and subconsciousness. The logic of consciousness is intuitionistic (we have, mainly, feelings about things, like about the truth of Conjectures in Mathematics, as an example), and the reunion between consciousness and subconsciousness is a conservative extension of consciousness (that is, the subconsciousness does not alter our awarness). So, the logic of human thinking is very similar to the logic of infons - see section 8., having as models non-standard objects from non-standard enlarged topoi - see section 8. again (based on sections 5.,6., and 7. also). From a mathematical point of view, is necessary to understand how the (quantum)neural networks from the brain (synapses) are influenced by the genetic-epigenetic (quantum) Turing mashine. The Quantum Physics approach is better to be based on truth values than on probabilities, the truth values-approach being used in topoi (see [6]). And, another important thing. A Theorem of Mathematics is based on the work of many others, so it is a by-product of the global brain. The Global Brain includes all the human brains AND the technology, used both for getting information from the outside of the direct human perception, and for connecting the brains (libraries, social networks,...). In this case the genetic-epigenetic interaction is between the Global Brain and the Environment, generating a kind of Global Plasticity for the Global Brain. Some details concerning (quantum) neural networks and (quantum) Turing machines will be enclosed in the second part of this work (II. Artificial Intelligence).We end the section with an example from Romania.

In 1966, Nicolae Ceausescu forbid abortion and contraception in order to increase the country 's population and work force. The birth rate raised rapidly, but many families could not afford having so many children. Thus, more and more orphanages were built, and by 1989 almost 170000 children lived in them. Here children were deprived of any sensorial stimulation or affection and everything was mechanized. They had the same haircut, were dressed the same, it was like a regiment. Doctor Charles Nelson observed these institutions and evaluated 136 children between 6 months and 3 years old. He found out that their IQ level was between 60 and 80, instead of 100. Even though their physiological needs were satisfied, their brain and language were underdeveloped. He discovered as well that their EEGs showed a dramatically reduced neural activity. What is more interesting is that if the children were moved as soon as possible in a safer environment full of love and affection, they could recover this delay. This study wonderfully emphasizes the role of epigenetics in our lives. Genetics is not enough for a proper development. The information we get through our sensory organs influences the way our DNA manifests. We see that babies touch and lick almost everything. By doing so, they better interact with the environment and improve their sight, orientation and perception of height, depth and three-dimension in 
general. Even though at birth the baby has the potential to learn any language and pronounce any sound, after hearing a certain language he/she will lose the ability to hear sounds of other languages. Every aspect of the world a child is raised in will sooner or later have an impact in his life.

\section{Connections with Physics: String Theory and Emergent Quantum Mechanics}

Certainly, everything happens in our real world, our real Universe, or our real Multiverse, or... This means that the human brain, human being, global brain,... represent substructures of the highest structure which must exist. There are discussions on what this highest structure is. We recall here the Theory of Multiverses belonging to Max Tegmark (see [31]). Namely, he classifies the notions of Multiverse in 4 categories: Multiverses of ranks 1, 2, 3, and 4. First of all, what is an Universe. In these theories the Universe is, by definition, the visible Universe. It is a 3D - sphere having its radius of about 14 billion light-years (the approximate period that has passed from the Big-Bang). This sphere is increasing as time passes. A rank 1 Multiverse is a family of Universes which could merge in some future in a bigger Universe. However, the General Relativity does not forbid that the space dilate with a speed higher than the speed of light. This means that two rank 1 Multiverses separated by a volume dilating faster than the speed of light will never merge at the level of a visible Universe. Such a family of rank 1 Multiverses is a rank 2 Multiverse. Because the evolutions are independent to a certain extent, the Universal constants, and even some of the laws of Physics among the rank 1 Multiverses belonging to the rank 2 Multiverse might differ. Further, there are discussions concerning the interpretations of Quantum Physics. One interpretation is based on the Copenhagen Program, based on the collapse of the waves of probabilities when measured (observed), and another one belongs to Hugh Everett III ([10]) saying that the probability waves never collapse, but what happens is in fact a bifurcation (multifurcation) of the (rank 2) Multiverse depending on all the possible situations (so, Schrodingers cat is alive in a Multiverse and dead in another one, at the same time). This is the so-called many-world interpretation, giving the Tegmark' rank 3 Multiverse (as a quantum family of rank 2 multiverses). And, finally, a rank 4 Multiverse is represented by pure Mathematics, with the Axiom saying that any non-contradictory mathematical theory should have real model(s) in the rank 4 Multiverse. This tries to explain why our Universe allows life to exist (all the Universal constants should be very, very carefully chosen: the number of Avogadro, the speed of light, the Planck distance or Planck time,...). We will restrict ourselves to a rank 3 Multiverse, as being the quantum family of our observable Universes. While the quantum logic of John von Neumann is considered as being the quantum logic in the Universe (in the sense of the previous definition), the quantum logic in topoi is considered as being the quantum logic in the Quantum Multiverse (rank 3). This is the logic which we will use, because it is the most appropriate to the human thinking, which is a product of the brain, a substructure of the Uni(Multi)verse (considered one of the most complex such substructures, along with black holes and dark matter). Coming back to the rank 4 Multiverse, it is based on the postulate that there are physical models for any non-contradictory mathematical theory. However, the best theories from physics, namely the Einstein' Theory of (General) Relativity (modeling the Universe at the macro-level), and the Quantum Physics (modeling the smallest sub-structures on the Uni(Multi)verse, at the nano-, pico-,... level) are in contradiction in some situations (the quantum entanglement is one of 
them). At the same time, the two theories should coexist in black holes, for instance. So, a new unifying theory is required, that is a theory unifying the Standard Model (which unifies the Electromagnetic, Strong, and Electroweak forces), a quantic model, with the Gravitation (modeled by the General Relativity). There are currently several approaches in this direction: String Theory, Emergent Quantum Mechanics, Loop Gravity,... We will restrict ourselves to some comments relative to the first two of them.

In String Theory (see [12]) one considers that any elementary particle is a tiny vibrating chord - string - in a 10-dimensional space, representing the direct product between the Einsteinian 4-dimensional space-time Universe (Einstein-Minkowski real 4-fold) and a complex (projective algebraic) Calabi-Yau 3-fold (so a 6-dimensional real manifold). The Calabi-Yau 3-fold is considered because it has very many symmetries, giving the so-called supersymmetric String Theory, modeling not only bosons (as the initial String Theory did) - that is particles with integer spin, but the fermions as well - particles with half integer but not integer spin. In such a situation the number of elementary particles (quarks, fotons, electrons,...) doubled, and one still look for the new elementary particles at CHERN, in Geneve. The 6 additional real dimensions of the Universe (Multiverse) are very compacted, and give only very limited possibilities to the string to vibrate, imposing conditions, as wave lenght, amplitude,... So, a string needs energy, in order to vibrate, and information, in order to vibrate in a specific way to give one or another of the elementary particles (or antiparticles). In [30] one considers hypothetical elementary particles related to information, calling them infons. It is for the first time when the notion has been considered. However, our point of view is based on the fact that these infons represent only one facet of an elementary particle, the other being represented by energons, so an elementary particle is like a coin, with two facets: the infon, and the energon. The theory of infons has been already discussed a little in section 2. After [30], a theory of infons (propositional calculus) has been built by [13]. They came to the conclusion that this theory is a conservative extension of the intuitionistic logic. However, one can show that the axiomatic of non-standard analysis in an environment using intuitionistic (many-valued) logic represents another similar notion of infon which we will use in this paper. So an infon is given here by the triplet $\mathcal{I}=(\mathcal{L}, \mathcal{A}, \mathcal{D})$, where $\mathcal{L}$ is the usual language of the intuitionistic non-standard context (see section 8.), $\mathcal{A}$ is a set of propositions written in the previous language considered as being true (axioms), and $\mathcal{D}$ is a set of deduction rules (as modus ponens is). For details on theories and categories, with application to the previous concrete case see sections 5.- 8. In our sense, the theory of infons is a metatheory given by the theory of non-standard enlargements of the topoi of type SET ${ }^{\mathcal{C}^{o p}}$ (see section 8.). In String Theory, an infon is a non-standard enlargement of a topos of the previous type, while in Emergent Quantum Mechanics an infon remains the theory itself, and the enlargement of the topos of the previous type (see Part II. Artificial Intelligence) will be called recepton. In String Theory we also need to define the energon. There are some tentatives, but:

In Emergent Quantum Mechanics [32]) one considers that the (potential) Energy is also Information, namely the information that something can move from one place to another. However, we think that we must distinguish between potential (virtual) energy and actual energy (kinetic, caloric, electromagnetic,...). The matter is considered a condensed form of (actual) energy (under the action of the strong nuclear force) related to energy by the well-known formula $E=m c^{2}$ (both the nuclear 
fission, from the atomic power plants and the nuclear fusion, from the Sun, generates loss of mass, producing energy). But a stronger argument connecting the (potential) energy with information is represented by The Landauer Principle, quantumising the energy necessary to erase a bit of information (for details see [4]). It means that both energy and information are quantised, and they are somehow inverse proportional (not exponential, as in the case of entropy, see section 2., but in the same direction, the relation being given by a precise formula, namely $E_{(\min )}=k_{B} T \ln (2)$, where $k_{B}$ is the Boltzmann constant, $T$ is the temperature of the environment and $l n$ is the natural logarithm. So, in Emergent Quantum Mechnics everything primordially exists as waves of informations $=$ theories in a conservative extension of the intuitionistic logic, all of them being of infon-type. Their associated categories (of models) represent then the other facets of the coin, called receptons so, in this setting the two faces are the infon (= a theory in the non-standard enlergement of the intuitionistic logic), and the recepton, the category of models of that theory (which is a non-standard enlargement of a topos of a special type, see sections 6.7., and section 2. from Part II). We, the human beings, are, at the level of our brains, or to the level of the global brain (which include both all the network of the human brains, and the technological discoveries, artificial intelligence included, but also other kind of sensory-type machines) like the radio-sets travelling in a landscape with plenty of electromagnetic waves, and discovering them progressively, function of the increasing quality of the receptor, improved due to the technologies. So, we become progressively aware of the surrounding informational waves (theories) improving progressively our consciousness (both at the individual and global level). At the same time, the subconsciousness works, impacting at every step on the consciousness. So, the receptons become the elementary pseudoparticles of the union between consciousness and subconsciousness, the two facets of the coins being the theory and the associated category (which must be a non-standard topos). In this version of a unification theory, the Uni(QuantumMulti)verse is emerging, being stepwise discovered by brain and global brain through the measurement (collapse) of the intuitionistic informational waves. In fact, Emergent Quantum Mechanics is essentially based on information and consciousness, but there are other important other facts around, namely the non-determinism (with the meaning that not only the past influences us, but also the future), the discrete space, Gosset Polytope, E8-crystal, and golden ratio $=\Phi=\lim _{n \rightarrow \infty}\left(F_{n+1} / F_{n}\right)$, where $\left(F_{n}\right)_{n \in \mathbf{N}}$ is the Fibonacci sequence. The $4 \mathrm{D}$ space-time is "pixelated" by the projection on $\mathbf{R}^{4}$ of the Gosset polytope from $\mathbf{R}^{8}$ with an angle which produce on $\mathbf{R}^{4}$ two similar 4-dimensional polytopes such that the quotient of their 4-volumes is equal to the golden ratio $(=(1+\sqrt{2}) / 2)$. We'll come back to Gosset polytope in section 8. In the Emergent Quantum Mechanics the consciousness become part of Physics. Both theories, String Theory, and Emerging Quantum Mechanics, are huge and complicated theories, but we do not insist here more that we already did it.

Starting with the next section, we will formalize in a mathematical language some of the previous comments. The formalism aims to produce a new mathematical approach for the Problems of Neuroscience. A list with problems from the aria is contained in the book [28]. 


\section{Theories versus Categories; Summary on the Category SET in Classical Logic}

For more details concerning the topics from this section, we refer to [5], [24], [27].

Definition 5.1. A class is an arbitrary collection of objects.

Remark 5.2 (Russel). Fixing two primary relations, namely $\in$ and $=$, which applies to classes, with the usual meaning (see 5.10), all the paranthesis: $(,[,\{\}]$, ), and the logic connectors: $\vee, \wedge, \neg, \rightarrow, \leftrightarrow$ and quantifiers $\forall, \exists$ with the usual meaning in a bivalent logic (see 5.9). Then the class

$$
\mathcal{M}=\{c \mid c \text { class } \wedge c \notin c, c \notin c=\neg(c \in c)\}
$$

is contradictory $(\mathcal{M} \in \mathcal{M} \leftrightarrow \mathcal{M} \notin \mathcal{M}$ is true $)$.

Definition 5.3 (Language).

1. $\mathcal{L}$-terms: Fix $x_{1}, x_{2}, \ldots$ variables. Then the $\mathcal{L}$-terms are:

- the variables;

- the classes;

- pairs, $t$-uples of the previous ones (we recall that $(a, b):=\{a,\{a, b\}\}$ and $\left(a_{1}, \ldots, a_{n}\right)=$ $\left(\left(a_{1}, \ldots, a_{n-1}\right), a_{n}\right)$ for any $\left.n \geq 3\right)$;

- if $\tau, \sigma$ are $\mathcal{L}$-terms, so is $\tau(\sigma)$.

2. $\mathcal{L}$-formulae:

- atomic: $\tau=\sigma, \tau \in \sigma$, with $\tau, \sigma, \mathcal{L}$-terms.

- then inductive, using:

- any atomic formula is a $\mathcal{L}$-formula;

- of $\varphi, \psi$ are $\mathcal{L}$-formulae, then so are $\varphi \wedge \psi, \varphi \vee \psi, \neg \varphi, \varphi \rightarrow \psi, \varphi \leftrightarrow \psi$;

- if $\varphi$ is a $\mathcal{L}$-formula, then so are $(\forall x \in \tau) \varphi$ and $(\exists x \in \tau) \varphi$, where $\tau$ is any $\mathcal{L}$-terms and $x$ any variable not appearing in $\tau$ :

3. A language $\mathcal{L}$ is the family of all formulae from before.

If $\mathcal{N}$ is a family of classes stable to " $\epsilon^{\prime \prime}$ (that is $\forall y\left(y \in \mathcal{N} \wedge x \in y \rightarrow x \in \mathcal{N}\right.$ ), then we denote by $\mathcal{L}_{\mathcal{N}}$ the restriction of $\mathcal{L}$ to $\mathcal{N}$ (this means that we replace in 1. all the classes with those classes from $\mathcal{N}$ only).

Definition 5.4. A sentence (or proposition) is a formula having all the variables bounded (cuantified). A sentence is true or false (bivalent logic, see 5.9).

Definition 5.5. A set of axioms is a set $\mathcal{A}$ of sentences, supposed true.

Definition 5.6. The deduction rules are the rules of inferrence from a set $\mathcal{D}$.

\section{Examples 5.7.}

1. modus ponens (MP): $\frac{p, p \rightarrow q}{q}$; 
2. particularization $(\mathrm{P}): \frac{\forall x p(x)}{p(y)}$;

3. generalization $(\mathrm{G}): \frac{p(y)}{\forall x p(x)}$, etc.

Definition 5.8. A theory is a triplet $\mathcal{T}=(\mathcal{L}, \mathcal{A}, \mathcal{D})$, with $\mathcal{L}, \mathcal{A}, \mathcal{D}$ as in 5.3, 5.5, 5.6 respectively; in the classical context (see 8.3 also) $\mathcal{A}$ contains strictly the axioms of $C L$ and $Z F(C)$ (see the next 5.9 and 5.10), with the only exception given by 5.10 (when $\mathcal{A}$ coincides with $C L$ and $Z F(C)$ ). We assume here, by definition, that a theory has at least one model. We note that, for example, if in 5.3.,3 we take $\mathcal{L}=\mathcal{L}_{\mathcal{U}(T)}($ for $\mathcal{U}(T)$ see 5.16) it is known that if this $\mathcal{L}$ is of order $n \geq 2$ (see 5.17) it is not true than any non-contradictory theory has a model, only for $n=1$ (first order language) this condition being automatically satisfied in general, for non-contradictory theories (see 5.19), this caracterising them, for $n=1$. As an example, the so-called $\omega$-inconsistent theory is not a theory in the sense of this definition.

Definition 5.9. A classical logic (CL) is a theory having the following axioms:

Then $-1: A \rightarrow(B \rightarrow A)$.

Then $-2:(A \rightarrow(B \rightarrow C)) \rightarrow((A \rightarrow B) \rightarrow(A \rightarrow C))$.

And $-1: A \wedge B \rightarrow A$.

And $-2: A \wedge B \rightarrow B$.

And $-3: A \rightarrow(B \rightarrow(A \wedge B))$.

Or $-1: A \rightarrow A \vee B$.

Or $-2: B \rightarrow A \vee B$.

Or $-3:(A \rightarrow C) \rightarrow((B \rightarrow C) \rightarrow(A \vee B \rightarrow C))$.

Not $-1:(A \rightarrow B) \rightarrow((A \rightarrow \neg B) \rightarrow \neg A)$.

Not $-2: A \rightarrow(\neg A \rightarrow B)$ (or $\perp \rightarrow B, \perp$ false).

Not $-3: A \vee \neg A$.

Definition 5.10. A set is a class which is an element of another class. Let's denote by $\mathcal{M}$ the class of sets. The theory of sets is the triplet $\left(\mathcal{L}_{\mathcal{M}}, C L+Z F(C), \mathcal{D}=\{M P\}\right)$, where $\mathrm{CL}$ are the axioms from 5.9, MP is as in 5.7, and ZF (C) (Zermelo-Fraenkel (+ choice)) are the following axioms (recall that " $\in$ " and "=" are primary relations):

1. Extensionality $(\mathbf{E})$ :

$$
\forall x \forall y[\forall z(z \in x \leftrightarrow z \in y) \rightarrow x=y]
$$

2. Regularity $(\mathbf{R})$ :

$$
\forall x[\exists a(a \in x) \rightarrow \exists y(y \in x \wedge \neg(\exists z(z \in y \wedge z \in x)))]
$$

3. Specification $(\mathbf{S})$ :

If $\varphi$ is a formula in $\mathcal{L}_{\mathcal{M}}$, with the free variables among $z, \omega_{1}, \ldots, \omega_{n}$ and having $y$ as a bounded variable:

$$
\forall z \forall \omega_{1} \forall \omega_{2} \ldots \forall \omega_{n} \exists y \forall x[x \in y \leftrightarrow((x \in z) \wedge \varphi)]
$$


In particular there are sets of the form $\{x \mid \varphi(x)\})$; note that this axiom allow us to consider such kind of sets only if they are subsets of a bigger set, avoiding in such a way the Russel' paradox from 5.2. Moreover, this axiom allow us to built the empty set, specifically

Remark 5.11. $\emptyset=\{x \in y \mid x \in x \wedge \neg(x \in x)\}$ (the empty set). Moreover, the empty set is unique (does not depend on $y$ ) by the Axiom 1.(extensionality).

4. Pairing $(\mathbf{P})$ :

$$
\forall x \forall y \exists z(x \in z \wedge y \in z)
$$

5. Union (U):

$$
\forall \mathcal{F} \exists A \forall Y \forall x[(x \in Y \wedge Y \in \mathcal{F}) \rightarrow x \in A]
$$

6. Replacement $(\mathbf{R p})$ :

For $\varphi$ a formula having $x, y, A, \omega_{1}, \ldots, \omega_{n}$ as free variables, then

$$
\forall A \forall \omega_{1} \forall \omega_{2} \ldots \forall \omega_{n}[\forall x(x \in A \rightarrow \exists ! y \varphi) \rightarrow \exists B \forall x(x \in A \rightarrow \exists y(y \in B \wedge \varphi))]
$$

(so, given the domain of a function, we can built its codomain); here $\exists$ ! means "there is a unique".

7. Infinity (I) (Peano):

Let's denote $S(\omega):=\omega \cup\{\omega\}$ (the successor of $\omega$, cf. (P) with $x=y=\omega$, and (U)):

$$
\exists X[\emptyset \in X \wedge \forall y(y \in X \rightarrow S(y) \in X]
$$

Definition-Remark 5.12. $\mathbb{N}:=$ the smallest $X$ as before (= the set of natural numbers), cf. Definition 5.13, from the next 8 .

8. Power set (PS):

Definition 5.13. $(z \subseteq x) \stackrel{\text { def }}{\Leftrightarrow} \forall q(q \in z \rightarrow q \in x)$.

Then:

$$
\forall x \exists y \forall z(z \subseteq x \rightarrow z \in y)
$$

Notation 5.14. $\mathrm{ZF}=$ the axioms $1 .-8 . \mathrm{ZFC}=$ the axioms $1 .-9$., where

9. Choice $(\mathbf{C})$ :

$$
\forall X\left[\emptyset \notin X \rightarrow\left(\exists f: X \rightarrow \bigcup_{A \in X} A\right) \wedge(\forall A \in X(f(A) \in A))\right.
$$

Theorem 5.15. If $\mathrm{ZF}$ the following are equivalent:

(i) (C);

(ii) any non-empty set can be well ordered (that is, any non-empty part has a minimum);

(iii) if any totally ordered subset of an ordered set has a majorant, then there exists a maximal element (Zorn' lemma).

Definition 5.16. Let $T$ be a set (possibly $T=\emptyset$ ). Then $\mathcal{U}=\mathcal{U}(T):=\bigcup_{n=0}^{\infty} U_{n}(T)$, where $U_{0}(T)=T$ and $U_{n+1}(T)=U_{n}(T) \cup \mathcal{P}\left(U_{n}(T)\right), \forall n \geq 0$. This is the Universe generated by $T$ (one can consider a bigger Universe, where the union is taken over all the ordinals, by transfinite recurrence - the von 
Neumann Universe - but we don't need it now). It is constructed from the set $T$, using $Z F$ axioms. We remark here that, even when $T=\emptyset$, we have $0=\emptyset \in U_{0}$, and, if $\omega \in U_{n}$, then $S(\omega)=\omega \cup\{\omega\} \in U_{(n+1)}$, so $\mathbb{N} \subset \mathcal{U}(\emptyset)$. Then, take $T=\mathbb{N}$. We get that all the number fields $(\mathbb{R}, \mathbb{C})$ are contained in $\mathcal{U}(N)$, by the definition uf $\mathcal{U}(N)$ and the $Z F$ axioms, using the constructions remembered in 7.1 - 7.15. Going further we get that $\mathbb{R}, \mathbb{C}$ (real and complex number fields) belongs to $\mathcal{U}(\mathbb{R})$. Continuing, it is easy to see that, in fact, all the necessary mathematical structures can be progressively built starting with the empty set $\emptyset$ (whose existence is postulated by $Z F$ ), uzing the $Z F(C)$ axioms.

Definition 5.17. A language $\mathcal{L}_{\mathcal{U}}$, associated with the previous Universe $\mathcal{U}$ can be defined as in 5.3, considering as $\mathcal{L}_{\mathcal{U}}$ - terms the variables, the elements of $\mathcal{U}, n$-uples, and $\tau(\sigma)$, where $\sigma$ and $\tau$ are as before, and continuing as in 5.3. If the quantifiers applies to $U_{0}(T)=T$ only, we call the resulting language, denoted by $\mathcal{L}_{T}$ a first order language, if the quantifiers applies to $U_{1}(T)$ only, we call the resulting language a second order language, denoted by $\mathcal{L}_{U_{1}(T)}, \ldots$,if the quantifiers applies to $U_{n-1}(T)$ only, we call the resulting language a $n$-th order language,...., in this terminology, $\mathcal{L}_{\mathcal{U}}$ being called a higher order language.

Examples of Theories 5.18. $\mathcal{T}=(\mathcal{L}, \mathcal{A}, \mathcal{D})$.

1. The theory of sets: $(\mathrm{Set})=(\mathcal{L}, C L+Z F, M P)$;

2. The theory of groups: $\mathcal{G}=(\mathcal{L}, \mathcal{A}=Z F C+C L+1)-4), M P)$, where:

1) $*: G \times G \rightarrow G$, function;

2) $\forall x \forall y \forall z[(x * y) * z=x *(y * z)]$;

3) $\exists e \forall x(x * e=e * x=x)$;

4) $\forall x \exists x^{\prime}\left(x * x^{\prime}=x^{\prime} * x=e\right)$.

3. The theory of rings: $\left.\left.\mathcal{R}=\left(\mathcal{L}, \mathcal{A}=Z F C+C L+1^{\prime}\right)-8^{\prime}\right), M P\right)$, where:

$\left.1^{\prime}\right)+: R \times R \rightarrow R$ function;

$\left.2^{\prime}\right) \cdot: R \times R \rightarrow R$ function;

$\left.3^{\prime}\right) \forall x \forall y \forall z[(x+y)+z=x+(y+z)]$;

$\left.4^{\prime}\right) \forall x \forall y \forall z[(x \cdot y) \cdot z=x \cdot(y \cdot z)]$;

$\left.5^{\prime}\right) \exists 0 \forall x(x+0=0+x=x)$;

$\left.6^{\prime}\right) \exists 1 \forall x(1 \cdot x=x \cdot 1=1)$;

$\left.7^{\prime}\right) \forall x \exists x^{\prime}\left(x+x^{\prime}=x^{\prime}+x=0\right)$;

8) $\forall x \forall y \forall z[x \cdot(y+z)=x \cdot y+x \cdot z]$;

$\left.9^{\prime}\right) \forall x \forall y(x+y=y+x)$.

If $\left.\left.\left.1^{\prime}\right)-9^{\prime}\right)+10^{\prime}\right) \forall x \exists x^{\prime}\left(\neg(x=0) \wedge x \cdot x^{\prime}=x^{\prime} \cdot x=1\right)$ one gets the theory of fields (in English the word field also presumes $\left.11^{\prime}\right) \forall x \forall y(x \cdot y=y \cdot x)$ ).

4. The theory of vector spaces over a field $\left.\left.\mathbb{K}: \mathcal{V}=\left(\mathcal{L}, Z F C+C L+1^{\prime \prime}\right)-10^{\prime \prime}\right), M P\right)$, where:

$\left.1^{\prime \prime}\right)+: V \times V \rightarrow V$ functions;

$\left.2^{\prime \prime}\right) \cdot: \mathbb{K} \times V \rightarrow V$ functions;

$\left.3^{\prime \prime}\right) \forall x \forall y \forall z[(x+y)+z=x+(y+z)]$;

$\left.4^{\prime \prime}\right) \exists 0 \forall x(x+0=0+x=x)$;

$\left.5^{\prime \prime}\right) \forall x \exists x^{\prime}\left(x+x^{\prime}=x^{\prime}+x=0\right)$; 


$$
\begin{aligned}
& \left.6^{\prime \prime}\right) \forall x \forall y(x+y=y+x) ; \\
& \left.7^{\prime \prime}\right) \forall x(1 \cdot x=x), 1=\mathbf{1}_{\mathbb{K}} \in \mathbb{K} ; \\
& \left.8^{\prime \prime}\right) \forall \lambda, \mu \in \mathbb{K} \forall x \in V[(\lambda+\mu) \cdot x=\lambda \cdot x+\mu \cdot x] ; \\
& \left.9^{\prime \prime}\right) \forall \lambda \in \mathbb{K} \forall x, y \in V[(\lambda \cdot(x+y)=\lambda \cdot x+\lambda \cdot y] ; \\
& \left.10^{\prime \prime}\right) \forall \lambda, \mu \in \mathbb{K} \forall x \in V[(\lambda, \mu) \cdot x=\lambda \cdot(\mu \cdot x)] .
\end{aligned}
$$

5. The theory of ordered sets: $\left.\left.\mathcal{O}=\left(\mathcal{L}, Z F C+C L+1^{\prime \prime \prime}\right)-4^{\prime \prime \prime}\right), M P\right)$, where:

$\left.1^{\prime \prime \prime}\right) \leq \subseteq \mathcal{O} \times \mathcal{O}$ subset;

$\left.2^{\prime \prime \prime}\right) \forall x(x \leq x)$;

$\left.3^{\prime \prime \prime}\right) \forall x \forall y(x \leq y \wedge y \leq x \rightarrow x=y)$

$\left.4^{\prime \prime \prime}\right) \forall x \forall y \forall z(x \leq y \wedge y \leq z \rightarrow x \leq z)$; etc.

Theorem 5.19. A first order theory (this means that its language is of first order, see 5.17) is non-contradictory iff it has at least one model (non-contradictory means that the false proposition is not deductible; a model is a concrete example of an object defined by the theory).

\section{Examples 5.20.}

1. $(\mathbb{Z},+)$ is a model for the theory of groups;

2. $(\mathbb{Z},+, \cdot)$ is a model for the theory of rings; $(\mathbb{Z}=$ the set of integer numbers $)$

3. $\left(\mathcal{M}_{n}(\mathbb{R}),+, \cdot\right)$ is a model for the theory of rings (non-commutative) (here $\mathcal{M}_{n}(\mathbb{R})$ represent the set of squared $n \times n$ matrices, and,$+ \cdot$ are the usuals addition and multiplication of matrices);

4. $\left(\mathcal{M}_{p q}(\mathbb{R}),+, \cdot\right)$ is a model for the theory of $\mathbb{R}$-vector spaces $\left(\mathcal{M}_{p q}(\mathbb{R})=\right.$ matrices with $p$ lines and $q$ columns, + being the usual addition, and - the scalar multiplication);

5. $(\mathbb{N}, \mid)$ is a model for the theory of ordered sets $(\mathbb{N}=$ the set of natural numbers, $a \mid b=a$ divides $b$ ).

6. More recently, it was observed that the set of all matrices is a model for the theory of (non-commutative) rings (see [16]). This ring can be interpretated as polinomials with matrices as variables, with $0,1 \in \mathbb{R}$, and the usual matrix product when it is possible to multiply matrices (otherwise the product is 0 ).

Definition 5.21. The family of all the models (or of a set of models) of a theory, together with the "dictionaries" between them (functions, or other objects, compatible with the structure of the theory) gives a category $\mathcal{C}_{\mathcal{T}}$ associated to the theory $\mathcal{T}$.

\section{Examples 5.22.}

1). Gr (category of groups): contains all (or a set of) groups, denoted by $O b(\mathbf{G r})$, and the morphisms between them, that is $\mathrm{Hom}_{G r}(G, H)=\left\{f: G \rightarrow H \mid f\left(x *_{G} y\right)=f(x) *_{H} f(y), \forall x, y \in G\right\}$.

2). Ring (category of rings): $O b$ (Ring) = a class (or set) of rings, $\operatorname{Hom}_{\text {Ring }}(R, S)=\{f: R \rightarrow$ $S \mid \forall x \forall y[f(x+y)=f(x)+f(y)] \wedge[f(x \cdot y)=f(x) \cdot f(y)]\}, f\left(1_{R}\right)=1_{S}$. Similarly for fields (the category Field), vector spaces (the category Vect), ordered sets (the category POSET), comming from 3.,4.,5. of 5.18 . 
Definition $5.23 \quad$ (Theory of Categories). $\quad A \quad$ category $\mathcal{C}$ is a 4-uple $\left(O b(\mathcal{C}),\left(\operatorname{Hom}_{\mathcal{C}}(A, B)\right)_{A, B \in O b(\mathcal{C})}, \circ,\left(\mathbf{1}_{X}\right)_{X \in O b(\mathcal{C})}\right)$ satisfying the axioms:

(i) $\circ: \operatorname{Hom}_{\mathcal{C}}(A, B) \times \operatorname{Hom}_{\mathcal{C}}(B, C) \longrightarrow \operatorname{Hom}_{\mathcal{C}}(A, C)$

$(f, g) \longmapsto g \circ f$ such that $(h \circ g) \circ f=h \circ(g \circ f) \forall f, g, h$ for which all the compositions are defined;

(ii) $\forall X \forall Y, X, Y \in O b(\mathcal{C}), \forall f \in H_{\mathcal{C}}(X, Y)$, the following diagrams are commutative:

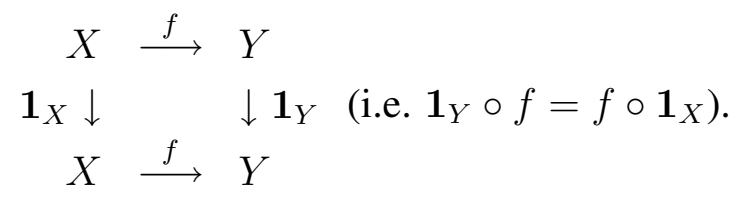

Remark 5.24. Any category (the models of a theory) is a model of the theory of categories, as are, for instance, the examples 5.22.

Remark 5.25. The category associated to the theory of categories is a category of categories, named CAT, that is:

$\mathrm{Ob}(\mathbf{C A T})=$ categories

$\operatorname{Hom}_{\mathbf{C A T}}\left(\mathcal{C}_{1}, \mathcal{C}_{2}\right)=$ the functors $\left(\right.$ see 5.26) from $\mathcal{C}_{1}$ to $\mathcal{C}_{2}$, with "o" and $\mathbf{1}_{\mathcal{C}}$ defined canonically.

Definition 5.26. Let $\mathcal{C}_{1}$ and $\mathcal{C}_{2}$ be two categories. A functor $F: \mathcal{C}_{1} \rightarrow \mathcal{C}_{2}$ is acting on objects: $F(A) \in$ $O b\left(\mathcal{C}_{2}\right), \forall A \in O b\left(\mathcal{C}_{1}\right)$ and on morphisms: $F(f) \in \operatorname{Hom}_{\mathcal{C}_{2}}(F(X), F(Y)), \forall f \in \operatorname{Hom}_{\mathcal{C}_{1}}(X, Y)$, being compatible with compositions and identities, that is:

(i) $F(g \circ f)=F(g) \circ F(f), \forall f, g$ such that the composition is defined, and

(ii) $F\left(\mathbf{1}_{X}\right)=\mathbf{1}_{F(x)}, \forall X \in O b\left(\mathcal{C}_{1}\right)$.

Remark 5.27. The previous definition introduce both the notion of morphism in CAT, and the theory of functors also. So, one expects to get the Category of functors also. Indeed,

Definition 5.28. Let $\mathcal{C}_{1}$ and $\mathcal{C}_{2}$ be two categories. We define the category $\mathcal{C}_{2}^{\mathcal{C}_{1}}$ as follows:

- $O b\left(\mathcal{C}_{2}^{\mathcal{C}_{1}}\right)=\left\{F: \mathcal{C}_{1} \rightarrow \mathcal{C}_{2} \mid F\right.$ functor $\}$

- $\operatorname{Hom}_{\mathcal{C}_{2}^{\mathcal{C}_{1}}}\left(F_{1}, F_{2}\right)=\left\{h: F_{1} \rightarrow F_{2} \mid h\right.$ is a natural transformation $\}$.

The composition and the identities are defined canonically.

Here we need the following:

Definition 5.29. If $F_{1}, F_{2} \in O b\left(\mathcal{C}_{2}^{\mathcal{C}_{1}}\right)$, a natural transformation $h: F_{1} \rightarrow F_{2}$ is a collection $h=\left(h_{X}\right)_{X \in O b\left(\mathcal{C}_{1}\right)}$ of morphisms $h_{X}: F_{1}(X) \rightarrow F_{2}(X), h_{X} \in \operatorname{Hom}_{\mathcal{C}_{2}}\left(F_{1}(X), F_{2}(X)\right)$, $F_{1}(X) \stackrel{h_{X}}{\longrightarrow} F_{2}(X)$

$\forall X \in O b\left(\mathcal{C}_{1}\right)$ such that $\forall f \in \operatorname{Hom}_{\mathcal{C}_{1}}(X, Y)$ the diagrams $F_{1}(f) \downarrow \quad \downarrow F_{2}(f)$ commutes (i.e. $\left.h_{Y} \circ F_{1}(f)=F_{2}(f) \circ h_{X}\right)$.

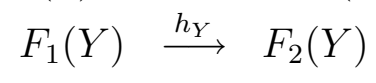

Remark 5.30. We insisted till now to get categories from theories, but the converse also works, axiomatizing the things. So, the theories and the categories are the two faces of the same thing, but they 
represent different notions.

Definition 5.31. Let $\mathcal{C}$ be a category. The dual category of $\mathcal{C}$, denoted by $\mathcal{C}^{o p}$ is the category having:

- $O b\left(\mathcal{C}^{o p}\right)=O b(\mathcal{C})$;

- $\operatorname{Hom}_{\mathcal{C}^{o p}}(X, Y)=H_{\mathcal{C}}(Y, X), \forall X, Y \in O b(\mathcal{C})$ (reversing the arrows).

Definition 5.32. A functor $F: \mathcal{C}_{1} \rightarrow \mathcal{C}_{2}^{o p}$ is called a cofunctor $F: \mathcal{C}_{1} \rightarrow \mathcal{C}_{2}$. It satisfies $F(g \circ f)=F(f) \circ F(g), \forall f, g$ (instead of $F(g \circ f)=F(g) \circ F(f), \forall f, g$ as a functor does). This is the first example of a pair of dual notions (functor vs. cofunctor). We'll see other examples in section 6.

\section{Examples 5.33.}

1. SET $=$ the category of sets.

$O b($ SET $)=$ sets.

$\operatorname{Hom}_{\mathbf{S E T}}(X, Y)=\{f: X \rightarrow Y \mid f$ functions $\}$.

2. $\mathbf{S E T} \mathbf{T}_{1}=$ the category of functions.

$O b\left(\mathbf{S E T}_{1}\right)=$ functions

$\operatorname{Hom}_{\mathbf{S E T}_{\mathbf{1}}}(f, g)=2$ - functions, namely

$$
\begin{array}{rlr}
X & \stackrel{f}{\longrightarrow} & Y \\
H_{\operatorname{mom}_{\mathbf{1}}}(f, g)=\{(\alpha, \beta) \mid \alpha \downarrow & & \downarrow \beta, \alpha, \beta \text { functions, and } \beta \circ f=g \circ \alpha\} \\
Z & \stackrel{g}{\longrightarrow} & Y
\end{array}
$$

3. $\mathbf{S E T}_{2}=$ the category of 2-functions.

$O b\left(\mathbf{S E T}_{2}\right)=2$ - functions.

$\operatorname{Hom}_{\mathbf{S E T}_{\mathbf{2}}}((\alpha, \beta),(\gamma, \delta))=4$ - functions, that is

$H_{\text {orm }}$ SET $_{\mathbf{2}}((\alpha, \beta),(\gamma, \delta))=\{(u, v, w, z) \mid u, v, w, z$ functions, such that all the squares of the following diagram $\mathbf{D}$ commutes $\}$.

\section{Diagram D :}

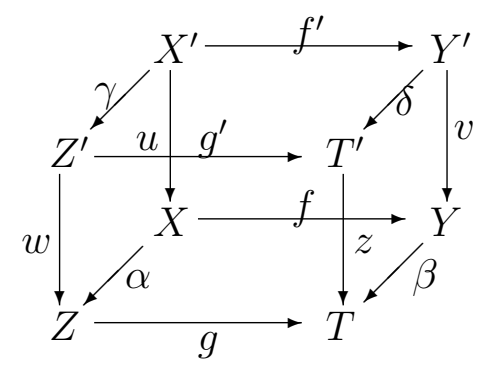

Continuing, we get (after obtaining 1D, 2D, 3D cubes, respectively, as morphisms, and 0D, 1D, 2D cubes, respectively, as objects) 4D cubes as morphisms (the 4D cube projected on the plane can be represented by a smaller cube inside a bigger one, with edges canonically connected) and 3D cubes as objects in $\mathbf{S E T}_{3}$, 5D cubes (two cubes in cubes one near another, canonically connected) as morphisms and 4 D cubes as objects in $\mathbf{S E T}_{4}$, and so on. Inductively, one can define $\mathbf{S E T}_{n}$ for any $n \geq 1$.

Definition 5.34. We call the category $\mathbf{S E T}_{n}$ the n-hypercube category. We'll come back to such examples in section 6 .

4. SET ${ }^{2}: O b\left(\mathbf{S E T}^{2}\right)=\{(A, B)=\{A,\{A, B\}\} \mid A, B \in O b(\mathbf{S E T})\}$ 
$H_{\text {om }}{ }_{\mathbf{S E T}}((A, B),(C, D))=\{(f, g) \mid f: A \rightarrow C, g: B \rightarrow D$ functions $\}$.

The composition and the identities are defined canonically.

5. SET ${ }^{n}: O b\left(\mathbf{S E T}^{n}\right)=\left\{\left(A_{1}, \ldots, A_{n}\right) \mid A_{1}, \ldots, A_{n}\right.$ sets $\}$

$\operatorname{Hom}_{\mathbf{S E T}^{n}}{ }^{n}\left(\left(\mathrm{~A}_{1}, \ldots, A_{n}\right),\left(B_{1}, \ldots, B_{n}\right)\right)=\left\{\left(f_{1}, \ldots, f_{n}\right) \mid f_{i}: A_{i} \rightarrow B_{i}\right.$ functions $\left.i=\overline{1, n}\right\}$.

Definition 5.35. The categories of type $\mathcal{C}_{2}^{\mathcal{C}_{1}}$ from the definition 5.28 are called exponential categories, and the categories of type 2. - 5. from Examples 5.33 are called of SET - type (see section 6 also).

6. SET/X $: O b(\mathbf{S E T} / \mathbf{X})=\left\{f \mid f \in H_{\mathrm{Som}}(A, X)\right.$ for some $A \in O b($ SET $\left.)\right\}$,

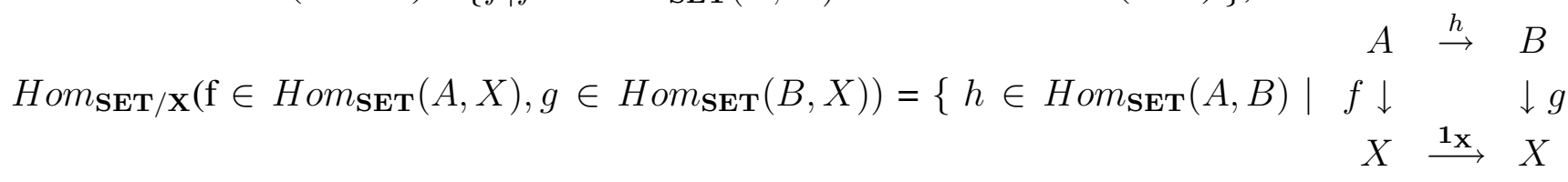
$\mathrm{g} \circ h=f\}$.

We'll come back to these examples in section 6.

7. TOP: $O b($ TOP $)=$ topological spaces

$H_{\text {om }}{ }_{\text {TOP }}(X, Y)=$ the continuous functions $: \mathrm{X} \rightarrow Y$.

Here we have:

Definition 5.36. A pair $(X, \tau)$, where $\tau \subset \mathcal{P}(X)$ (the power-set of $X)$ is a topological space, if:

(i) $\phi \in \tau, X \in \tau$;

(ii) $\forall\left(D_{i}\right)_{i \in I}$ such that $D_{i} \in \tau, \forall i \in I$, then $\bigcup_{i \in I} D_{i} \in \tau$;

(iii) $\forall n \forall D_{1} \ldots \forall D_{n}, D_{1}, \ldots, D_{n} \in \tau$, then $D_{1} \cap \ldots \cap D_{n} \in \tau$.

Example 5.37. $\mathbb{R}^{n}$ with the usual topology of metric space (given by the Euclidian norm).

Definition 5.38. $f:\left(X, \tau_{X}\right) \rightarrow\left(Y, \tau_{Y}\right)$, with $\left(X, \tau_{X}\right)$ and $\left(Y, \tau_{Y}\right)$ topological spaces is continuous if $\forall x \in X \forall D_{Y} \in \tau_{Y} \exists D_{x} \in \tau_{X}\left(x \in D_{x} \wedge f(x) \in D_{Y} \wedge\left(f\left(D_{x}\right) \subseteq D_{Y}\right)\right)$.

Examples 5.39. All the elementary functions $f: \mathbb{R}^{n} \rightarrow \mathbb{R}^{m}$ (polynomial, exponential, trigonometric,... ) are continuous.

8. POSET (partially ordered sets):

$\mathrm{Ob}($ POSET $)=$ partially ordered sets $(=$ the models of the theory 5 from example 5.18).

$\operatorname{Hom}_{\text {POSET }}\left(\left(X, \leq_{X}\right),\left(Y, \leq_{Y}\right)\right)=\left\{f: X \rightarrow Y \mid \forall x \forall y x \leq_{X} y \rightarrow f(x) \leq_{Y} f(y)\right\}$ (the increasing functions).

Remark 5.40. In the classification of N. Bourbaki, the fundamental structures are the algebraic ones (as Gr, Ring, Vect, Field, ... ), POSET and TOP, all the others (including the differential ones) being combinations of these 3 classes of structures.

We end this section with a special kind of POSET $=$ Algebraic structure, related to the theory of sets (ZF) and the bivalent logic (CL), namely the Boolean latice (or the Boolean algebra). 
Definition 5.41. A Boolean Algebra is a set $A$, together with 3 operations and 2 symbols, that is $(A,+,-, 0,1)$, with $+: A \times A \rightarrow A, \cdot: A \times A \rightarrow A,-:$ A $\rightarrow A$ satisfying
a) $\forall x \forall y(x+y=y+x) ; \forall x \forall y:(x \cdot y=y \cdot x)$;
b) $\forall x \forall y \forall z[(x+y)+z=x+(y+z)] ; \forall x \forall y \forall z[(x \cdot y) \cdot z=x \cdot(y \cdot z)]$;
c) $\forall x \forall y \forall z[x \cdot(y+z)=x \cdot y+x \cdot z] ; \forall x \forall y \forall z[x+(y \cdot z)=(x+y) \cdot(x+z)]$;
d) $\forall n(x+0=0+x=x) ; \forall n(x \cdot 0=0 \cdot x=0)$;
e) $\forall n(x+1=1+x=1) ; \forall n(x \cdot 1=1 \cdot x=x)$;
f) $\forall n(x+\bar{x}=\bar{x}+x=1) ; \forall n(x \cdot \bar{x}=\bar{x} \cdot x=0)$;
g) $\forall x(\overline{\bar{x}}=x)$;
h) $\forall x \forall y(\overline{x+y}=\bar{x} \cdot \bar{y}) ; \forall x \forall y(\overline{x \cdot y}=\bar{x}+\bar{y})$.

Remark 5.42. Some of the previous axioms can be deducted from others, but we preferred to include them all. Other deductible sentences are:

i) $\forall x(x+x=x) ; \forall x(x \cdot x=x)$;

j) $\forall x \forall y[x \cdot(x+y)=x] ; \forall x \forall y[x+(x \cdot y)=x]$.

Definition 5.43. A poset $(X, \leq)$ for which there exists $x \wedge y=\inf \{x, y\}, x \vee y=\sup \{x, y\}$, it is bounded (i.e. $0 \leq x \forall x, \exists 1, n \leq 1, \forall n)$, complemented (i.e. $\forall n \exists n^{\prime}\left[x \wedge x^{\prime}=0 \wedge\left(x \vee x^{\prime}=1\right) \wedge\left(x^{\prime \prime}=x\right)\right]$ and, if we replace in the Definition $5.41 x+y$ by $x \vee y$ and $x \cdot y$ by $x \wedge y$, all the properties of 5.41 are satisfied for the corresponding $\wedge$ and $\vee$, we say that the poset $\left(A, \wedge, \vee,^{\prime}, 0,1\right)$ is a Boolean lattice.

Remark 5.44. The notions of Boolean algebra and Boolean lattice are interchangeable via the correspondences $(+, \vee),(\cdot, \wedge),\left(-,^{\prime}\right),(0,0),(1,1)$ where, given a Boolean algebra we get on it a poset structure putting $x \leq y \stackrel{\text { def }}{\Leftrightarrow} x \cdot y=x$.

Example 5.45. 1. $T$ set, then $(\mathcal{P}(T), \subseteq)$ is a Boolean lattice $\left(0=\emptyset, 1=T, \vee=\cup, \wedge=\cap,^{\prime}=\right.$ the complementary).

2. $\{f:\{1, \ldots, n\} \rightarrow\{0,1\} \mid f$ function $\}$ can be considered as strings of the form 001010011100 of $n$ letters, the order being given by the order of numbers considered as being written in the base 2. 000000000000 is the smallest element, 111111111111 is the lowest element, the addition, the multiplication, and the negation are done on components, where: $0+0=0,0+1=1+0=1+1=1$, and $0.0=0.1=1.0=0,1.1=1,0^{\prime}=1,1^{\prime}=0$. We get a Boolean algebra (finite, with $2^{n}$ elements)

Definition 5.46. Let $\mathcal{P}$ be the set of sentences, and let's denote by $\top$ the sentence always true $(p \vee \neg p)$ and by $\perp=\neg(\top)$ the sentence always false $(p \wedge \neg p)$. A function $\mathbf{V}: \mathcal{P} \rightarrow B=$ Boolean algebra is called a $B$-valuation if:

$\mathbf{V}(\top)=1, \mathbf{V}(\perp)=0, \mathbf{V}(p \wedge q)=\mathbf{V}(p) \cdot \mathbf{V}(q)$

$\mathbf{V}(p \vee q)=\mathbf{V}(p)+\mathbf{V}(q), \mathbf{V}(\neg p)=\overline{\mathbf{V}(p)}$, where $\wedge, \vee, \neg$ are the logical operations, and $+, \cdot,-$ are the operations from $B$ (one can add, if we like, $\mathbf{V}(p \rightarrow q)=\overline{\mathbf{V}(p)}+\mathbf{V}(q)$, although it follows from the other requirements; we recall that $p \rightarrow q=\neg p \vee q$ ). 
Definition 5.47. If $F$ is a formula, we say that $F$ is valid if $\mathbf{V}(F)=1$ for any $\mathbf{V}: \mathcal{P} \rightarrow\{0,1\}$. We say that $F$ is $B$-valid if $\mathbf{V}(F)=1$ for any valuation $\mathbf{V}: \mathcal{P} \rightarrow B=$ Boolean algebra.

Theorem 5.48. If $F$ is a formula then $F$ is provable in CL (that means that it is deductible from the axioms by MP) $\Leftrightarrow F$ is $B$-valid for any Boolean algebra $B$.

Notations 5.49. If $\mathcal{T}=(\mathcal{L}, \mathcal{A}, \mathcal{D})$ is a theory, we write $\mathcal{T} \vdash \varphi$ if $\varphi$ is provable in the theory $\mathcal{T}$ (i.e. deductible from $\mathcal{A}$ using $\mathcal{D}$ ). We write $\mathcal{T} \models \varphi$ if $\varphi$ is true on any model of $\mathcal{T}$ (so, in the category $\mathcal{C}_{\mathcal{T}}$ ).

Theorem 5.50. (Gödel completness) For any first-order theory $(5.17,5.19)$

$$
\begin{gathered}
\mathcal{T} \vdash \varphi \Longleftrightarrow \mathcal{T} \models \varphi \\
\text { (semantic) } \quad(\text { sintactic })
\end{gathered}
$$

We'll see in section 6 a generalization of the Boolean algebras (lattices), namely Heyting algebras (lattices), related to the intuitionistic (multi-valuated) logic. The axioms of (CL) from 5.9 are verified on a Boolean algebra.

In the classical logic one uses sentences (propositions) - with the previous logical connectors $\vee, \wedge, \neg, \rightarrow,-$ but also predicates $p=p(x), p\left(x_{1}, \ldots, x_{n}\right)$ and quantifiers as $\forall$ and $\exists$ (universal and existential).

Definition 5.51. A monadic Boolean algebra (abreviated MBA) is an algebraic structure $(B,+, \cdot,-, 0,1, \exists, \forall)$, where $(B,+, \cdot,-, 0,1)$ is a Boolean algebra (see 5.41), and $\exists$ (resp. $\forall$ ) is closure (resp. interior) operator, that is:

(i) $\exists 0=0$;

(ii) $a \leq \exists a$, for any $a \in B$ (recall that $a \leq b$ iff $a \cdot b=a$, iff $a+b=b$, iff $a \rightarrow b=1$; we recall that $a \rightarrow b=\bar{a}+b)$;

(iii) $\exists a=\exists \exists a$, for any $a \in B$;

(iv) $\exists(a \vee b)=(\exists a) \vee(\exists b)$, for any $a, b \in B$.

(resp.

(i) ${ }^{\prime} \forall 1=1$;

(ii) ${ }^{\prime} \forall a \leq a$, for any $a \in B$;

(iii) ${ }^{\prime} \forall a=\forall \forall a$, for any $a \in B$;

(iv) ${ }^{\prime} \forall(a \wedge b)=(\forall a) \wedge(\forall b)$, for any $a, b \in B$.

Note. Here $a \wedge b=a \cdot b, a \vee b=a+b$ (we preferred to use the logic related symbols).

Remarks 5.52. 1). $\forall$ can be seen as a (possible) infinite conjuction; $\exists$ can be seen as a (possible) infinite disjunction.

2). Based on 1). and on De Morgan relations, one can see $\forall$ as being: $\forall x=\bar{\exists} \bar{x}$ (i.e. $\neg \forall x p(x)=$ $\exists x \neg p(x)$ and $\neg \exists x p(x)=\forall x \neg p(x)$, in logical symbols). So, in the Boolean case, the two operators $\forall$ and $\exists$ are deductible one from another. We'll see later (section 6) that this is not the case in the Heyting-algebras case. 
3). We built algebraic structures related to all the logical connectors necessary to formally define a language $\mathcal{L}$, as in 5.3.

\section{Summary on Topos Theory and its Intuitionistic Logic; Exponential and SET-type Topoi}

For more details concerning the topics from this section, we refer to [10], [25].

We start this section with the notion already announced at the end of the previous section, namely the Heyting lattice (or algebra), which is related to the intuitionistic (or multi-valued) logic (IL) as the Boolean lattice (or algebra) is related to the bi-valent logic (CL) from section 5. So

Definition 6.1. The axioms of (IL) are (notations from 5.9):

Then - 1, Then - 2;

And - 1, And - 2, And - 3;

Or - 1, Or - 2, Or - 3;

Not 2.

Definition 6.2. A poset $(H, \leq)$ is called a Heyting lattice if it is a bounded lattice (so, for any $a, b \in H$ there exists $a \wedge b=\inf \{a, b\}, a \vee b=\sup \{a, b\}$, there exists $0,1 \in H$ such that $0 \leq x \leq 1$ for any $x \in H$ ) satisfying the conditions a) - e) from 5.41, when replacing + and $\cdot$ by $\vee$ and $\wedge(0,1$ remain the same), and having an operation $\rightarrow$ satisfying:

$$
\forall a, b, c,(a \wedge b \leq x \Leftrightarrow a \leq(b \rightarrow c))
$$

So, a Heyting lattice is a 6-uple $(H, \vee, \wedge, \rightarrow, 0,1)$ as before. A Heyting algebra is a 6-uple $(H,+, \cdot, \rightarrow, 0,1)$ obtained by replacing $\vee$ and $\wedge$ with + and $\cdot$ Given a Heyting algebra we get from it a poset which is a Heyting lattice, putting $a \leq b \Leftrightarrow a \rightarrow b=1$ (see 5.51 (ii)).

Remark 6.3. The condition (1) from 6.2 is satisfied in the Boolean case for $b \rightarrow c=b^{\prime} \vee c$.

Remark 6.4. Any Heyting algebra (lattice) satisfies the axioms of (IL) from 6.1.

Examples 6.5. 1). All the Boolean algebras (lattices) are Heyting algebras (lattices).

2). The followings are Heyting algebras (lattices), but not Boolean algebras (lattices)

(i) $\left(\left\{0, \frac{1}{2}, 1\right\}, \wedge, \vee, \rightarrow, 0,1\right)$ (lattice) where:

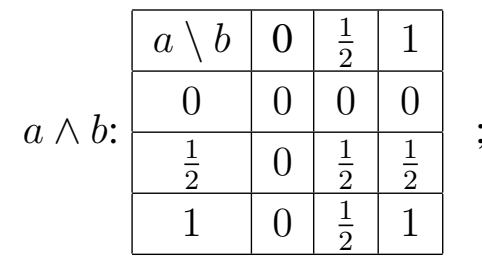

\begin{tabular}{|c|c|c|c|c|}
\hline \multirow{4}{*}{$a \vee b:$} & $a \backslash b$ & 0 & $\frac{1}{2}$ & 1 \\
\hline & 0 & 0 & $\frac{1}{2}$ & 1 \\
\hline & $\frac{1}{2}$ & $\frac{1}{2}$ & $\frac{1}{2}$ & 1 \\
\hline & 1 & 1 & 1 & 1 \\
\hline
\end{tabular}




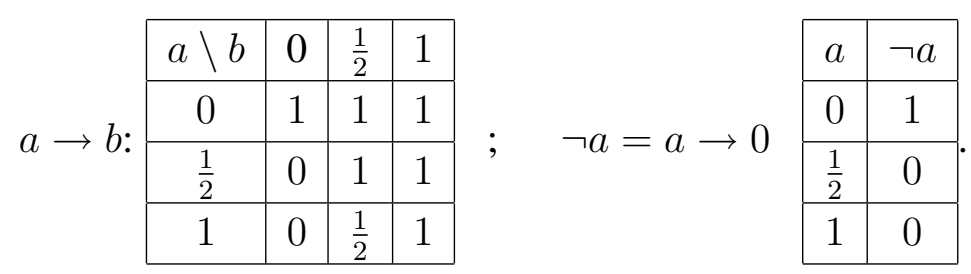

We have $\frac{1}{2} \vee \neg \frac{1}{2}=\frac{1}{2} \vee\left(\frac{1}{2} \rightarrow 0\right)=\frac{1}{2} \vee 0=\frac{1}{2}$, so the low of excluded middle is not satisfied (as it is in the Boolean case).

(ii) If $(X, \tau)$ is a topological space (5.33 example 7). If $A \subset X$, we define the interior of $A$ as being the set $\left\{a \in A \mid \exists D_{a} \in \tau\right.$ s.t. $\left.a \in D_{a} \subset A\right\}:=\stackrel{\circ}{A}$. Then $(X, \vee, \wedge, \rightarrow 0,1)$ is a Heyting algebra which is not Boolean in general, where: for $A, B \in \tau, A \vee B:=A \cup B \in \tau, A \wedge B:=A \cap B \in \tau$, $A \rightarrow B:=\stackrel{\circ}{B \backslash A} \in \tau, 0=\phi \in \tau, 1=X \in \tau$.

We'll see other examples in the context of topoi.

Definition 6.6. We can define a negation in Heyting algebra, putting $\neg a:=a \rightarrow 0$ (this is compatible with the Boolean case), but it does not satisfy the principle of double negation and other principles (tertium not datum, reductio ad absurdum, De Morgan laws in general (see example 2 (i) from (6.5))).

We'll meet the (IL) on the objects of some special categories, entitled topoi. We'll spend some time now with some complements on the theory of categories which are necessary to define the notion of topos and to built its logic. So:

In Section 5, we already introduced the notion of category (5.23), functor (5.26), cofunctor (5.32), dual category (5.31), natural transformation (5.29). We asserted that the notions of functor and cofunctor are dual, one notion arrising from the other by considering a dual category $\mathcal{C}^{o p}$, obtained by reversing the arrows of the initial category $\mathcal{C}$.

Let $\mathcal{C}$ be a category, $\mathcal{C}=\left(O b(\mathcal{C}),\left(\operatorname{Hom}_{\mathcal{C}}(A, B)\right)_{A, B \in O b(\mathcal{C})}, \circ,\left(\mathbf{1}_{X}\right)_{X \in O b(\mathcal{C})}\right)$.

Definition 6.7. A morphism $f: A \rightarrow B$ is called monomorphism if $\forall u_{i}: X \rightarrow A, i \in\{1,2\}$, such that $f \circ u_{1}=f \circ u_{2}$ it follows $u_{1}=u_{2}$.

Definition 6.7'. A morphism $f: A \rightarrow B$ is called epimorphism if $\forall u_{i}: B \rightarrow Y, i \in\{1,2\}$, such that $u_{1} \circ f=u_{2} \circ f$ it follows that $u_{1}=u_{2}$.

Remark 6.8. The two previous notions are dual to each other; they are obtained by reversing the arrows.

Remark 6.9. In the category SET (see 5.33, 1.) monomorphism is equivalent to injective and epimorphism with surjective.

Definition 6.10. A morphism $\varphi \in \operatorname{Hom}_{\mathcal{C}}(A, B)$ is called isomorphism iff $\exists \psi \in \operatorname{Hom}_{\mathcal{C}}(B, A)$ such that $\psi \circ \varphi=\mathbf{1}_{A}$ and $\varphi \circ \psi=\mathbf{1}_{B}$. In this case one says that the objets $\mathrm{A}$ and $\mathrm{B}$ are isomorphic (write $A \simeq B$ ). 
Remark 6.11. If it exists, the morphism $\psi$ is unique, and one writes $\psi=\varphi^{-1}$.

Examples 6.12. 1. In SET isomorphism $\Leftrightarrow$ bijective homomorphism.

2. In Gr, Rings, Vect isomorphism $\Leftrightarrow$ bijective homomorphism.

3. In Top a bijective continous map is not necessarly isomorphism (the inverse might not be continous), so one use exactly the definition 6.10 (the isomorphism is called homeomorphism) (see $5.22,5.33,7)$.

Definition 6.13. Let $X, Y \in O b(\mathcal{C})$. A triplet $\left(X \times Y, p_{X}, p_{Y}\right)$ with $X \times Y \in O b(\mathcal{C}), \quad p_{X} \in$ $H_{\mathcal{C}}(X \times Y, X), p_{Y} \in H o m_{\mathcal{C}}(X \times Y, Y)$ is called a direct product if for any diagram in $(Z, f, g)$ in $\mathcal{C}$ like the next one

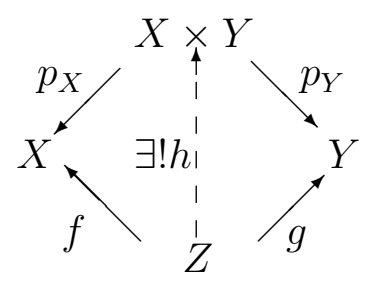

there is a unique $h=(f, g)$ (the dashed arrow) making the diagram commutative (i.e. $p_{X} \circ h=$ $f, p_{Y} \circ h=g$ ) (this the of universality of the direct product).

Definition 6.13'. The dual notion is called direct sum, $X+Y$.

Remark 6.14. Given the morphisms $f$ and $g$ as in the next diagram, we get, using 6.13 (the property of universality of the direct product) a unique $\mathrm{h}$ (the dashed arrow) such that $p_{Y} \circ h=f \circ p_{X}$ and $p_{T} \circ h=g \circ p_{Z}$. The morphism $h=f \times g$ is called the direct product of the morphisms $\mathrm{f}$ and $\mathrm{g}$.

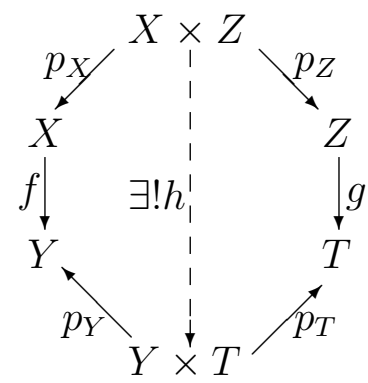

Dualizing, one gets the direct sum of morphisms, that is $f+g$.

Definition 6.15. An object $I \in O b(\mathcal{C})$ is called initial object in $\mathcal{C}$ if $\forall X \in O b \mathcal{C}, \exists ! I \rightarrow X$ (i.e. card $\left.\left(H_{\mathcal{C}}(I, X)\right)=1, \forall X \in O b(\mathcal{C})\right)$. Dualizing, one gets the notion of final object (or terminal object) in $\mathcal{C}$.

Remark 6.16. Any two direct products (or direct sums, initial objects and final objects, respectively) are isomorphic (this results from the so-called properties of universality appearing in the definitions of 
these objects; a similar isomorphism always exists when we have an universality).

Remark 6.17. In the category SET, the direct product is the cartesian product, the direct sum is the disjoint union, the initial object is the $\emptyset=$, the empty set, the final objects are (any of) the singletons $\{x\}$.

Definition 6.18. Given $f, g \in \operatorname{Hom}_{\mathcal{C}}(X, Y)$, we call the equalizer of $(f, g)$ the pair $(E, e)$, with $e$ a monomorphism, having the following property of universality: for any pair $(Z, h)$ such that $f \circ h=g \circ h$ there is a unique $k \in \operatorname{Hom}_{\mathcal{C}}(Z, E)$ with $e \circ k=h$, as in the following diagram :

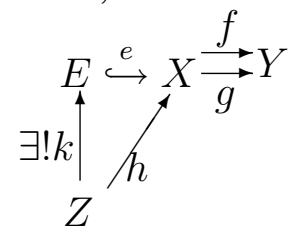

Definition 6.18'. Dualizing, we get the notion of coequalizer of $(f, g)$.

Remark 6.19. In the category SET, $E=\{x \in X \mid f(x)=g(x)\}$ with the inclusion $E \hookrightarrow X$ is an equalizer. The coequalizer also exists in SET, although it is more difficult to define it.

Definition 6.20. 1). One calls a diagram $\mathcal{D}$ in $\mathcal{C}$ a collection of objects and morphisms between them: $\mathcal{D}=\left\{\left(X_{i}\right)_{i \in I}, X_{i} \in O b(\mathcal{C}),\left(g=g_{i, j}^{k} \in \operatorname{Hom}_{\mathcal{C}}\left(X_{i}, X_{j}\right)\right)_{i, j \in I}\right\}$. For fixed $(i, j)$ it is possible to have more $g$ 's, or none (so the index $k=k(i, j)$ can be zero, one, or greater than one).

2). One calls a cone over $\mathcal{D}$ a pair $\left(Y,\left(f_{i}\right)_{i \in I}\right), Y \in O b(\mathcal{C}), f_{i} \in H_{\mathcal{C}}\left(Y, X_{i}\right)$ for any $\mathrm{i}$ (we can have more $f_{i}$, or none) such that all the following diagrams commute, for all $i, j \in I$, and all $g$ 's from the diagram $\mathcal{D}$ :

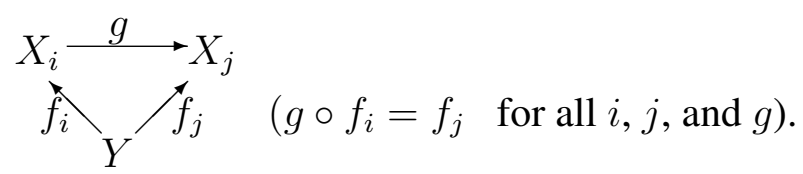

Definition 6.21. Let $\mathcal{D}$ be a diagram in $\mathcal{C}$. We call an inductive limit (or direct limit) for $\mathcal{D}$ a cone $\left(C,\left(f_{i}\right)_{i \in I}\right)$ such that any other cone $\left(C^{\prime},\left(f_{i}^{\prime}\right)_{i \in I}\right)$ uniquely factorises through it, this means that there is a unique $\mathrm{f}$ as in the following diagram, such that $f_{i} \circ f=f_{i}^{\prime}, \forall i$.<smiles>[Y][C@H](C)[CH][SiH3]</smiles>

Definitions 6.20'. Dualizing, one gets the dual notions of co-cone and projective (or inverse) limit.

Notations 6.22. For $\mathcal{D}$ any diagram, we denote the inductive (resp. projective) limits as $\underline{\lim } \mathcal{D}$ (resp. $\lim \mathcal{D})$.

Examples 6.23. $1 . \mathcal{D}=$ the diagram with 2 objects, $X_{1}$ and $X_{2}$, no arrow. Then a cone over $\mathcal{D}$ is given by the diagram : 


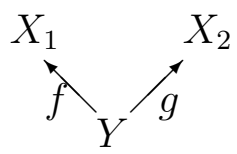

Then $\underset{\lim }{\longrightarrow} \mathcal{D}=X_{1} \times X_{2}$ (the direct product). Dually, $\lim _{\underline{D}} \mathcal{D}=X_{1}+X_{2}$ (the direct sum), see 6.13.

2. D: $f: X_{1} \rightarrow X_{2}, g: X_{1} \rightarrow X_{2}$. Then $\underline{\lim } \mathcal{D}=$ the equalizer of $(f, g)$ and $\lim \mathcal{D}=$ the co-equalizer of $(f, g)$, see 6.18 .

3. $\mathcal{D}$ : the empty diagram. Then $\lim _{\longrightarrow} \mathcal{D}=$ final object, and $\lim _{\underline{D}} \mathcal{D}=$ initial object, see 6.15 .

Definition 6.24. We call a pullback of $(f, g)$ the $\stackrel{\lim }{\longrightarrow} \mathcal{D}$, with $\mathcal{D}: X \stackrel{f}{\longrightarrow} Z \stackrel{g}{\longleftarrow} Y$. We call a pushout of $\left(f^{\prime}, g^{\prime}\right)$ the $\lim \mathcal{D}^{\prime}$, where $\mathcal{D}^{\prime}: X^{\prime} \stackrel{f^{\prime}}{\longleftarrow} Z^{\prime} \stackrel{g^{\prime}}{\longrightarrow} Y^{\prime}$ (these are dual notions).

Definition 6.25. A category $\mathcal{C}$ is called complete if $\exists \longrightarrow \lim \mathcal{D}$ for any diagram $\mathcal{D}$ in $\mathcal{C}$, co-complete if $\exists \lim \mathcal{D}$ for any diagram $\mathcal{D}$ in $\mathcal{C}$, finitely complete (resp. finitely co-complete) if the previous injective and projective limits exist for all finite diagrams $\mathcal{D}$ (including the empty ones).

Remark 6.26. From (6.22) and (6.23) it results that if a category $\mathcal{C}$ is finitely complete and finitely co-complete, then it has:

- initial and final objects;

- equalizer and co-equalizer of any $(f, g)$;

- direct products and direct sums;

- pullbacks and pushouts.

Example 6.27. One can check that the category SET is finitely complete and co-complete.

Definition 6.28. We say that a category $\mathcal{C}$ has exponentiation if it has direct products of any two objects and there is a pair $\left(Y^{X}, e v\right)$, with $Y^{X} \in O b(\mathcal{C})$, ev $\in H_{\mathcal{C}}\left(Y^{X} \times X, Y\right)$ satisfying the following property of universality: for any $Z \in O b(\mathcal{C})$, any $g \in H o m_{\mathcal{C}}(Z \times X, Y)$ there is a unique $\hat{g} \in H_{\mathrm{O}}\left(Z, Y^{X}\right)$ such that $e v \circ\left(\hat{g} \times \mathbf{1}_{X}\right)=g$ (for $\hat{g} \times \mathbf{1}_{X}$ see (6.14)), see the diagram below :

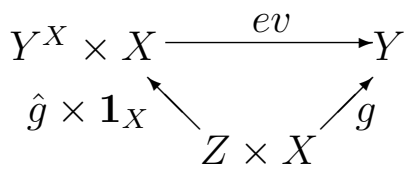

Example 6.29. For the category SET, if $A, B \in O b(\mathbf{S E T})$, then the pair $\left(B^{A}, e v\right)$ satisfies the previous property of universality, where

$$
\begin{gathered}
B^{A}=\{f \mid f: A \rightarrow B\}=H_{\mathrm{SET}}(A, B) \\
e v: B^{A} \times A \longrightarrow B
\end{gathered}
$$




$$
(f, x) \longmapsto f(x) .
$$

So the category SET has exponentiation.

Definition 6.30. Let's consider a fixed $d \in O b(\mathcal{C})$ and the pairs $(b, i)$ with $b \stackrel{i}{\hookrightarrow} d$ a monomorphism. We identify two such pairs $(b, i)$ and $\left(b^{\prime}, i^{\prime}\right)$ iff there are $\alpha \in H o m_{\mathcal{C}}\left(b, b^{\prime}\right), \beta \in \operatorname{Hom}_{\mathcal{C}}\left(b^{\prime}, b\right)$ such that $\alpha \circ \beta=\mathbf{1}_{b^{\prime}}$ and $\beta \circ \alpha=\mathbf{1}_{b}, i^{\prime} \circ \alpha=i$ and $i \circ \beta=i^{\prime}$ (so $b \simeq b^{\prime}$ in $\mathcal{C}$, see 6.10).

Let's denote by [b] these classes of isomorphisms of the pairs $(b, i)$ as before. Such a class [b] is called subobject of $d$. So, $S u b_{\mathcal{C}}(d)=\{[b] \mid(b, i)$ as before $\}$ represents the family of subobjects of $d$.

Definition 6.31. We define a partial order on $S u b_{\mathcal{C}}(d)$, as it follows:

Take two subobjects $[a],[b] \in S u b_{\mathcal{C}}(d)$ and let $a \stackrel{f}{\hookrightarrow} d$ and $b \stackrel{g}{\hookrightarrow} d$ be two representants. Write $f \leq g$ if there is a monomorphism $a \stackrel{\varphi}{\hookrightarrow} b$ such that $g \circ \varphi=f$. Then: $[a] \subseteq[b] \stackrel{\text { def }}{\Longleftrightarrow} f \leq g$.

One can check that the notion is well-defined and the relation is reflexive, anti-symmetric and transitive, so $\left(S u b_{\mathcal{C}}(d), \subseteq\right)$ becomes a poset.

Definition 6.32. Let $1 \in \mathcal{C}$ be a final object (we suppose that it exists). A pair $(\Omega, \top)$, with $\Omega \in O b(\mathcal{C})$ and $T \in \operatorname{Hom}_{\mathcal{C}}(1, \Omega)$ is called subobjects classifier if it satisfies the $\Omega$-axiom: that is: for any

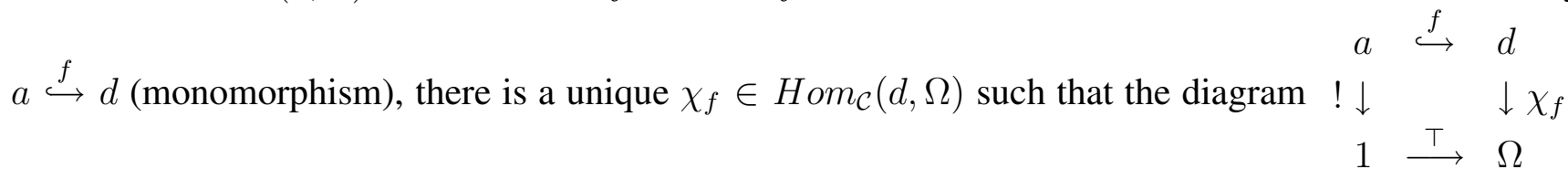
is commutative, i.e. $T \circ !=\chi_{f} \circ f$ (here $!$ is the unique morphism from $a$ to 1 , since 1 is a final object).

Example 6.33. In the category SET, $\Omega=\{0,1\},\{1\} \stackrel{\top}{\longrightarrow}\{0,1\}$ the natural inclusion map, and $\chi_{f}$ is the usual characteristic function.

Theorem 6.34. If $a \stackrel{g}{\hookrightarrow} d, b \stackrel{f}{\hookrightarrow} d$ are two monomorphisms, we have

$$
[f]=[g] \Longleftrightarrow \chi_{f}=\chi_{g}
$$

Proof. Standard, chasing on diagrams. Now, we can, finally, give the following

Definition 6.35. An elementary topos is a category $\mathcal{E}$ such that:

(T1) $\mathcal{E}$ is finitely complete (see 6.25)

(T2) $\mathcal{E}$ is finitely co-complete (see 6.25)

(T3) $\mathcal{E}$ has exponentiation (see 6.28)

(T4) $\mathcal{E}$ has subobjects classifier (see 6.32).

Examples 6.36. 1). From 6.27, 6.29, 6.33 it results that SET is a topos. Moreover, $\operatorname{card}\left(\operatorname{Hom}_{\mathbf{S E T}}(1, \Omega)\right)=2, \operatorname{Hom}_{\mathbf{S E T}}(1, \Omega)$ (with $1=$ singleton, final object), consisting (using the usual characteristic function) in $\{0<1\}, \Omega=\{0,1\}$ being the subobjects classifier. The morphisms from $\operatorname{Hom}_{\mathbf{S E T}}(1, \Omega)$ are called the global sections of $\Omega$. 
2). If $\mathcal{E}$ is a topos, $X \in O b(\mathcal{E})$ then $\mathcal{E} / X$ is a topos, where $O b(\mathcal{E} / X)=\{\alpha \mid \alpha \in$ $\left.\left.\operatorname{Hom}_{\mathcal{E}}(A, X)\right)_{A \in O b(\mathcal{E})}\right\}$ and

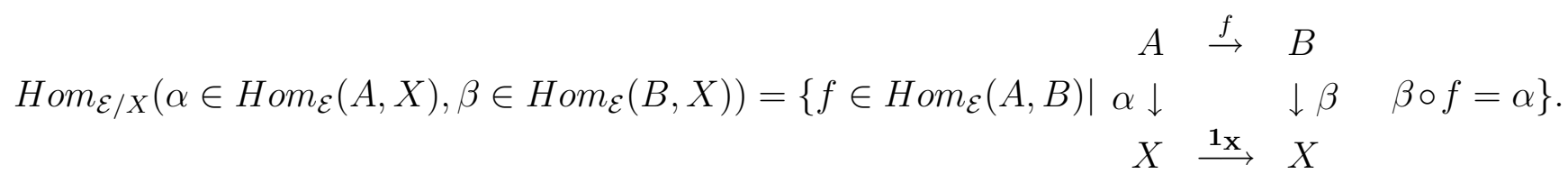

In particular one gets that SET $/ x$ is a topos (compare with 5.33, 6.)

3). SET ${ }^{n}: O b\left(\mathbf{S E T}^{n}\right)=\left\{\left(A_{1}, \ldots, A_{n}\right) \mid A_{i} \in O b(\mathbf{S E T}), i=\overline{1, n},(n\right.$-uples of sets $)$, and

$$
\operatorname{Hom}_{\mathbf{S E T}^{n}}\left(\left(A_{1}, \ldots A_{n}\right),\left(B_{1}, \ldots B_{n}\right)\right)=\left\{\left(f_{1}, \ldots, f_{n}\right) \mid f_{i} \in \operatorname{Hom}_{\mathbf{S E T}}\left(A_{i}, B_{i}\right), i=\overline{1, n}\right\} .
$$

is also a topos. In this case the finite completeness and co-completeness can be checked on components. For the exponentiation, we define $\left(A_{1}, \ldots, A_{n}\right)^{\left(B_{1}, \ldots, B_{n}\right)}:=\left(A_{1}^{B_{1}}, \ldots, A_{n}^{B_{n}}\right)$, with the evaluation morphism defined on components. The subobjects classifier is $\Omega=\underbrace{(\{0,1\}, \ldots,\{0,1\})}_{n \text {-times }}$, and the characteristic morphism is $\chi_{A}=\left(\chi_{A_{1}}, \ldots, \chi_{A_{n}}\right)$ for $A=\left(A_{1}, \ldots, A_{n}\right)$, where $\chi_{A_{i}}$ is the usual characteristic function from SET. Let's denote by 1 a (the) final object (see (6.15)) from this category, $1=\left(\left\{x_{1}\right\}, \ldots,\left\{x_{n}\right\}\right)$. Then any morphism $1 \rightarrow A$ is a monomorphism (see (6.7)) for any $A=\left(A_{1}, \ldots A_{n}\right) \in O b\left(\mathbf{S E T}^{n}\right)$. We remark that $\operatorname{card}\left(\operatorname{Hom}_{\mathbf{S E T}^{n}}(1, \Omega)\right)=2^{n}$.

4). $\operatorname{SET}^{n_{1}, \ldots, n_{s}}$, for $n_{1}<n_{2}<\ldots<n_{s}, s \in \mathbb{N}^{*}, n_{i} \in \mathbb{N}, i=\overline{1, n}$, and if $n_{1}=0$, then $A_{1}=\emptyset$.

$$
O b\left(\mathbf{S E T}^{n_{1}, \ldots, n_{s}}\right)=\left\{\left\{A_{1}, A_{2}, \ldots, A_{s}\right\} \mid A_{i} \in O b\left(\mathbf{S E T}^{n_{i}}\right), i=\overline{1, s}\right\}
$$

(see 3).). $H_{\text {om }}$ SET $^{n_{1}, \ldots, n_{s}}\left(\left\{A_{1}, \ldots, A_{s}\right\},\left\{B_{1}, \ldots, B_{s}\right\}\right)=\left\{\left\{f_{1}, \ldots, f_{s}\right\} \mid f_{i} \in \operatorname{Hom}_{\mathbf{S E T}^{n_{i}}}\left(A_{i}, B_{i}\right), i=\right.$ $\overline{1, s}$ (we remark that for any $\mathrm{A}_{i}$ there is a unique $B_{i}$, by renumbering the $B$ 's if necessary, having the same number of components as $A_{i}$, because $n_{1}<n_{2}<\ldots<n_{s}$ ). The compositions and identities are defined (both in 3). and 4).) in a canonical way. The exponentiation is defined as $\left\{A_{1}, \ldots, A_{s}\right\}^{\left\{B_{1}, \ldots, B_{s}\right\}}=\left\{A_{1}^{B_{1}}, \ldots, A_{n}^{B_{n}}\right\}$ where, as before, $\mathrm{A}_{i}$ and $B_{i}$ are chosen to belong to the same $O b\left(\mathbf{S E T}^{n_{i}}\right)$ and $A_{i}^{B_{i}}=\operatorname{Hom}_{\mathbf{S E T}^{n_{i}}}\left(A_{i}, B_{i}\right)$. The subobjects classifier object is

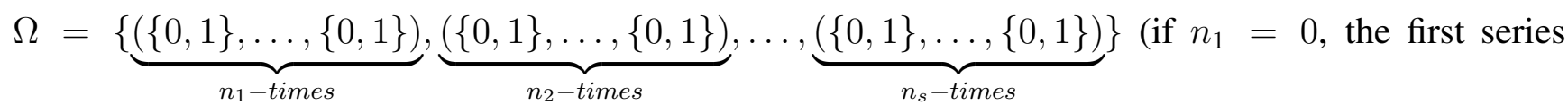

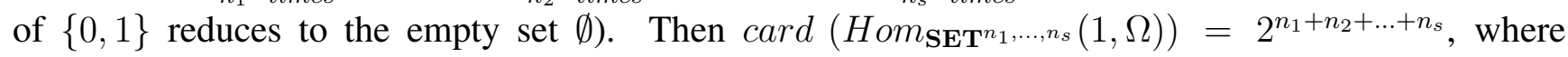
$1=\left\{\left(\left\{x_{11}\right\},\left\{x_{12}\right\}, \ldots,\left\{x_{1 n_{1}}\right\}\right),\left(\left\{x_{21}\right\},\left\{x_{22}\right\}, \ldots,\left\{x_{2 n_{2}}\right\}\right), \ldots,\left(\left\{x_{s 1}\right\},\left\{x_{s 2}\right\}, \ldots,\left\{x_{s n_{s}}\right\}\right)\right\}$ is a (the) final object in the category SET ${ }^{n_{1}, \ldots, n_{s}}$. We remark that the condition $n_{1}<n_{2}<\ldots<n_{s}$ makes this category similar with the categories from 3 )., but we will interpret it differently in section 8 .

5). Now we will come back to the categories of type 3., but we will consider some aditional conditions, requesting some inclusions among the sets $A_{i}$. For example we consider the category $\mathbf{S E T}_{3}^{8}$ whose objects are $O b\left(\mathbf{S E T}_{3}^{8}\right)=\left\{\left(A_{1}, \ldots, A_{8}\right) \mid A_{i} \in O b(\mathbf{S E T}), i=\overline{1,8}\right.$, and $\mathrm{A}_{1} \subset A_{2}, A_{3} \subset A_{4}, A_{1} \subset A_{3}$, $\left.A_{2} \subset A_{4}, A_{5} \subset A_{6}, A_{7} \subset A_{8}, A_{5} \subset A_{7}, A_{6} \subset A_{8}, A_{1} \subset A_{5}, A_{2} \subset A_{6}, A_{3} \subset A_{7}, A_{4} \subset A_{8}\right\}$ and the morphisms given by 8 -tuples of functions, as in 3 )., but compatible with all the inclusions (for example, 
with the notations from 3)., the function from $A_{1}$ to $B_{1}$ should be the restriction and corestriction of the function from $A_{2}$ to $B_{2}$, and similarly for all the inclusions). Note that this category is a kind of 3D cube, similar to the category $\mathrm{SET}_{3}$ from 5.33 (the points $\mathrm{X}^{\prime}, \mathrm{Y}^{\prime}, \mathrm{Z}^{\prime}, \mathrm{T}^{\prime}, \mathrm{X}, \mathrm{Y}, \mathrm{Z}, \mathrm{T}$ are replaced by the sets $A_{1}, \ldots, A_{8}$, and the arrows are replaced by inclusions). This time the subobjects classifier is, as in 3 )., $\Omega=\underbrace{(\{0,1\}, \ldots,\{0,1\})}_{8 \text {-times }}$, but $\operatorname{card}\left(\operatorname{Hom}_{\mathbf{S E T}_{3}^{8}}(1, \Omega)\right)=20$ (the global sections being lexicographically ordered) $\left\{t_{1}=(0,0,0,0,0,0,0,0)<t_{2}=(0,0,0,0,0,0,0,1)<t_{3}=(0,0,0,0,0,0,1,1)<\right.$ $t_{4}=(0,0,0,0,0,1,0,1)<t_{5}=(0,0,0,0,0,1,1,1)<t_{6}=(0,0,0,0,1.1,1,1)<t_{7}=$ $(0,0,0,1,0,0,0,1)<t_{8}=(0,0,0,1,0,0,1,1)<t_{9}=(0,0,0,1,0,1,0,1)<t_{10}=$ $(0,0,0,1,0,1,1,1)<t_{11}=(0,0,0,1,1,1,1,1)<t_{12}=(0,0,1,1,0,0,1,1)<t_{13}=$ $(0,0,1,1,0,1,1,1)<t_{14}=(0,0,1,1,1,1,1,1)<t_{15}=(0,1,0,1,0,1,0,1)<t_{16}=$ $(0,1,0,1,0,1,1,1)<t_{17}=(0,1,0,1,1,1,1,1)<t_{18}=(0,1,1,1,0,1,1,1)<t_{19}=$ $\left.\left.(0,1,1,1,1,1,1,1)<t_{20}=(1,1,1,1,1,1,1,1)\right\}\right)$ (use the characteristic morphism from 3)., and the fact that, for $\mathrm{X}$ and $\mathrm{Y}$ sets, $X \subset Y$ implies $\chi_{X} \leq \chi_{Y}$ ).

A simpler example is given by $\mathbf{S E T}_{2}^{4}$, whose objects are $\left\{\left(A_{1}, A_{2}, A_{3}, A_{4}\right) \mid A_{1} \subset A_{2}, A_{3} \subset A_{4}, A_{1} \subset\right.$ $\left.A_{3}, A_{2} \subset A_{4}\right\}$, with morphisms as in 3$)$., compatible with the existing inclusions. In this cases $\operatorname{card}\left(\operatorname{Hom}_{\mathbf{S E T}_{2}^{4}}(1, \Omega)\right)=6$, the sections being $\{(0,0,0,0)<(0,0,0,1)<(0,0,1,1)<(0,1,0,1)<$ $(0,1,1,1)<(1,1,1,1)\}$. Another example, connected with the Gosset' polytope considered in Emergent quantum mechanics, will be considered is section 8 .

We note that the categories $\mathbf{S E T}_{n}$ from 5.33, 2.,3. are also topoi and we can show this generalizing the previous arguments. For example for $\mathbf{S E T}_{1}$, fix an object $f: X \rightarrow Y$ from $O b\left(\mathbf{S E T}_{1}\right)$. Then, any subobject of $f$ is a function $g: A \rightarrow B$ such that $A \subset X, B \subset Y$ and $g$ is the restriction and corestriction of $f$ (we can take this subobject as a representant of the isomorphism class of monomorphisms to $f$, by 6.34). Then, for an $x \in X$ there are three possibilities : i) : $x \in A ; i i$ ) : $x \notin A, f(x) \in B ; i i i$ ) : $x \notin A, f(x) \notin B$ (when classifying the subobjects of $f$ we interpretate the monomorphisms as inclusions, and we get exactly the previous studied cases from the current example). So, there are 3 possibilities of appartenence, hence we can take the subobjects classifier as being $\Omega=\left\{\mathbf{1}_{\{0,1\}}:\{0,1\} \rightarrow\{0,1\}\right\}$, and $\phi_{g: A \rightarrow B}(x, g(x))=\left(\phi_{A}(x), \phi_{B}(g(x))\right)$. So, $\mathbf{S E T}_{1}$ is a topos with 3 truth values. Similarly, identifying $\mathbf{S E T}_{2}$ with the previous $\mathbf{S E T}_{2}^{4}$ and $\mathbf{S E T}_{3}$ with $\mathbf{S E T}_{3}^{8}$, we get topoi with 6 and 20 truth values, respectively. And we can continue with further (all) $\mathrm{SET}_{n}$.

6). We give the last exemple for now. It is of the type of topoi considered in 5)., but we put it separately, because it proves that for any natural number $n \geq 2$ there is a topos $\mathcal{E}$ such that $\operatorname{card}\left(\operatorname{Hom}_{\mathcal{E}}(1, \Omega)\right)=n$. So, let $m \in \mathbf{N}^{*}$ a positive natural number. We consider the category $\operatorname{SET}(m)$ whose objects are m-uples of increasing sets: $\operatorname{Ob}(\mathbf{S E T}(m))=\left\{\left(A_{1}, A_{2}, \ldots, A_{m}\right) \mid A_{1} \subset\right.$ $\left.A_{2} \subset \ldots \subset A_{m}\right\}$. The morphisms are as in 3), and compatible with the inclusions. Then the category $\operatorname{SET}(\mathbf{m})$ is a topos, such that $\operatorname{card}\left(\operatorname{Hom}_{\mathbf{S E T}}(m)(1, \Omega)\right)=m+1$ (with 1 and $\Omega$ as before), the global sections being $(0,0, \ldots, 0,0,0)<(0,0, \ldots, 0,0,1)<(0,0, \ldots, 0,1,1)<(0,0, \ldots, 1,1,1)<\ldots<$ $(0,1,1, \ldots, 1,1)<(1,1,1, \ldots, 1,1)$. 
6.37. The Heyting lattice on $S u b_{\mathcal{E}}(X), X \in O b(\mathcal{E}), \mathcal{E}$ topos (see 6.29, 6.30).

- (I): Intersection $(\cap)$ :

Let $a \stackrel{i}{\hookrightarrow} X, b \stackrel{j}{\hookrightarrow} X$ be two subobjects of $X$. Let $Y \in O b(\mathcal{E})$ the pullback of the diagram $a \stackrel{i}{\hookrightarrow} X \stackrel{j}{\longleftrightarrow}$ $b$, that is

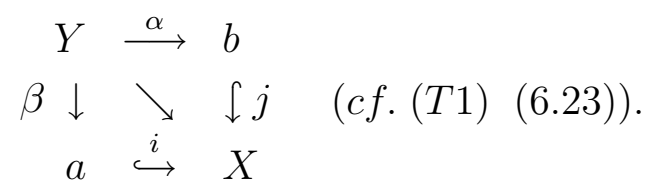

Since the pullback preserves the monomorphisms, it means that $\alpha$ and $\beta$ are monomorphisms also, so $j \circ \alpha=i \circ \beta$ is again a monomorphism. This construction is independent of the representents $a \in[a]$, $b \in[b]$ (in the sense that for other representents we get an $Y^{\prime} \simeq Y$ (canonically). Then

Definition 6.38. $[a] \cap[b]:=[Y]$ (or $[i] \cap[j]=[j \circ \alpha=\beta \circ i])$.

- $(\mathrm{U})$ : Union $(\cup)$ :

We first construct the image of an arrow $A \stackrel{f}{\longrightarrow} B$, denoted by $\operatorname{Im} f$. For this, we consider the pushout $A \stackrel{f}{\rightarrow} B$

of the diagram $B \stackrel{f}{\longleftarrow} A \stackrel{f}{\longrightarrow} B$, denoted by $P$, that is $f \downarrow \quad \downarrow y \quad$ (cf. 6.35 (T2) and (6.24)).

$B \stackrel{x}{\longrightarrow} P$

Let now $m=$ the equalizer of $(x, y): M \rightarrow B$, (cf. 6.35 (T1), 6.23, 2, 6.26). (It easy to see that $m$ is a monomorphism).

Since $x \circ f=y \circ f$, it follows that $f$ factorize uniquely through $M$, via $e$ :

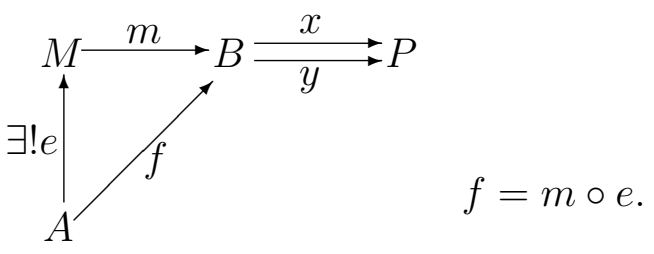

(due to the property of universality of the equalizer). So, we get the diagram

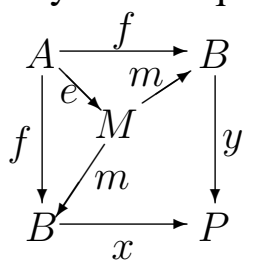

It can be checked that $m$ is a monomorphism, $e$ is epimorphism, and $f=m \circ e$.

Definition 6.39. Im $f: \stackrel{\text { def }}{=} M$.

And now, let's define the union.

Let $a \stackrel{i}{\hookrightarrow} X, b \stackrel{j}{\hookrightarrow} X$ two subobjects of $X$. Write

Put $E:=\operatorname{Im}(i+j)$ (cf. 6.13', 6.39, $6.35\left(T_{2}\right)$, and 6.26). Then $a \cup b:=E($ or $i \cup j=\operatorname{Im}(i+j)$ ). The construction depends only a $[a]=[i]$ and $[b]=[j]$. So, we get the following

Definition 6.40. $[a] \cup[b]=[\operatorname{Im}(i+j)]$ (or $[i] \cup[j]=[\operatorname{Im}(i+j)]$ ).

- (Im): Implication $(\rightarrow)$ :

Let's consider first a terminal object $1 \in O b(\mathcal{E})$, and look to $S u b_{\mathcal{E}}(1)$. This is a lattice (take $X=1$ in the previous two constructions), and let $a \stackrel{i}{\hookrightarrow} 1, b \stackrel{j}{\hookrightarrow} 1$ be two subobjects. $a, b \in O b(\mathcal{E})$ and by 6.35 (T3) there exists the exponential $a^{b} \in O b(\mathcal{E})$. It can be seen that the unique morphism $a^{b} \rightarrow 1$ is a monomorphism, so $\left[a^{b}\right] \in S u b_{\mathcal{E}}(1)$. The construction depends only on the isomorphism classes of 
$[a]=[i]$ and $[b]=[j]$, and we give now the following

Definition 6.41. $[a] \rightarrow[b]=\left[a^{b}\right] \in S u b_{\mathcal{E}}(1)$. For $X \in O b(\mathcal{E})$ general we have a bijection $S u b_{\mathcal{E}}(X) \simeq$ $\operatorname{Sub}_{\mathcal{E} / X}\left(1_{X}\right)$, where $1_{X}=\operatorname{Hom}_{\mathcal{E}}(1, X)$ with 1 from before. The previous bijection is an isomorphism of lattices also, and $\mathcal{E} / X$ is a topos also (see 6.36, 2.). Using this isomorphism, one can transfer the implication on $S u b_{\mathcal{E}}(X)$.

- If 1 is a final object then the top element of $S u b_{\mathcal{E}}(1)$ is the unique $\mathbf{1}_{1}: 1 \rightarrow 1$, and the bottom element is ! : $0 \rightarrow 1$, with 0 an initial object. One can check that $S u b_{\mathcal{E}}(1)$ becomes a Heyting lattice (6.2), as well as $S u b_{\mathcal{E}}(X)$, where $! X: 0 \rightarrow X$ and $\mathbf{1}_{X}: X \rightarrow X$ in the identity of $X$.

Remark 6.42. If $\mathcal{E}$ is a topos, then $S u b_{\mathcal{E}}(1)$ ( 1 a final object) and $S u b_{\mathcal{E}}(\Omega)$ ( $\Omega$ the subobjects classifier) are models of (IL) (see 6.1).

6.43. However, on $\Omega$ we can define logic connectors also, namely $\wedge, \vee, \rightarrow, \perp, \top(\top=$ true, $\perp=$ false $)$, as follows:

- $T: 1 \rightarrow \Omega$ is the arrow from the definition of the subobjects classifier (6.32).

$0 \stackrel{!}{\hookrightarrow} 1$

- $! \downarrow \quad \downarrow \chi$ ! is the diagram of the $\Omega$-axiom for $f=$ !, 0 initial object (6.32). Then $\perp:=\chi$ !

$1 \stackrel{\top}{\longrightarrow} \Omega$

- Apply now the $\Omega$-axiom to the diagram

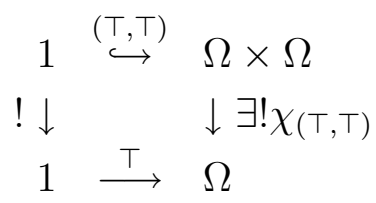

Then $\wedge: \Omega \times \Omega \rightarrow \Omega, \wedge:=\chi_{(\top, \top)}$.

- Let $\left(\top, \mathbf{1}_{\Omega}\right)+\left(\mathbf{1}_{\Omega}, \top\right): \Omega+\Omega \rightarrow \Omega \times \Omega$ and $M \stackrel{i}{\hookrightarrow} \Omega \times \Omega$ its image (see 6.39). Apply the $\Omega$-axiom to the diagram

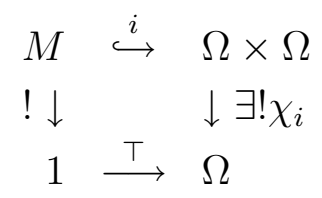

and put $\vee:=\chi_{i}$.

- Finally, let $e: \leq \hookrightarrow \Omega \times \Omega$ the equalizer (see 6.18) of $\wedge: \Omega \times \Omega \rightarrow \Omega$ and $p r_{1}: \Omega \times \Omega \rightarrow \Omega$, where $\wedge$ is the conjunction constructed before, and $p r_{1}$ is the first projection arrow of $\Omega \times \Omega \rightarrow \Omega$ (see 6.13). Then write the $\Omega$-axiom for

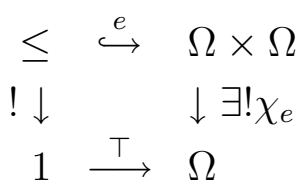

and put $\rightarrow:=\chi_{e}$.

Remark 6.44. One can define a negation also by the formula $\neg: \Omega \rightarrow \Omega, \neg \alpha:=\alpha \rightarrow 0$. This is compatible with the case of Boolean algebras, but it doues not satisfy the principle of double negation 
(and others), in general.

6.45. As in the Boolean case (see 5.46), we can define $\mathcal{E}$-valuations $\mathrm{V}: \mathcal{P} \rightarrow H \operatorname{Hom}_{\mathcal{E}}(1, \Omega)$ where $\mathcal{E}$ is a topos, $\mathcal{P}$ the set of sentences (see 5.4), 1 (the) final object, $\operatorname{Hom}_{\mathcal{E}}(1, \Omega$ ) all the monomorphisms of this kind, $T$ being one of them.

Definition 6.46. An $\mathcal{E}$-valuation is a function $\mathbf{V}_{0}: \mathcal{P}_{0} \rightarrow H o m_{\mathcal{E}}(1, \Omega)$ assigning each $\mathcal{L}$-term which is a sentence $\pi_{i} \in \mathcal{P}_{0}$ (see 5.3), a truth value $\mathbf{V}\left(\pi_{i}\right): 1 \rightarrow \Omega$. Then extend $V_{0}$ to $\mathbf{V}: \mathcal{P} \rightarrow H_{\mathcal{E}}(1, \Omega)$ by the rules

a) $\mathbf{V}(\alpha \wedge \beta)=\wedge \circ(\mathbf{V}(\alpha), \mathbf{V}(\beta))$

b) $\mathbf{V}(\alpha \vee \beta)=\vee \circ(\mathbf{V}(\alpha), \mathbf{V}(\beta))$.

c) $\mathbf{V}(\alpha \rightarrow \beta)=\rightarrow \circ(\mathbf{V}(\alpha), \mathbf{V}(\beta))$.

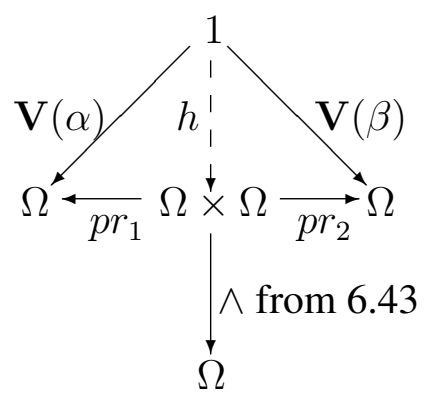

Here the logic symbols from the left members are those coming from the propositional calculus, while the others come from the constructions from 6.43.

It also results that $\mathbf{V}(\neg \alpha)=\neg \circ \mathbf{V}(\alpha)$.

Definition 6.47. For $\mathcal{E}$ topos, we say that $\alpha \in \mathcal{P}$ is $\mathcal{E}$-valid, denoted by $\mathcal{E} \models \alpha$, iff $\mathbf{V}(\alpha)=\top: 1 \rightarrow \Omega$, for any $\mathcal{E}$-valuation $\mathbf{V}$.

Theorem 6.48. Let $\mathcal{E}$ be a topos, and write $C L \vdash \alpha$ (resp. $I L \vdash \alpha$ ) for $\alpha \in \mathcal{P}$, if $\alpha$ is deductible from the axioms of the classical logic, see 5.9 (resp. $\alpha$ is deductible from the axioms of the intuitionistic logic, see 6.1). Then:

1. If $\mathcal{E} \models \alpha$ then $C L \vdash \alpha$.

2. If $\mathcal{E}$ is Boolean (that is $\operatorname{card}\left(\operatorname{Hom}_{\mathcal{E}}(1, \Omega)\right)=2$ ) then $\mathcal{E} \models \alpha$ iff $C L \vdash \alpha$.

3. (completeness) $\mathcal{E} \models \alpha$ for any topos $\mathcal{E}$ iff $I L \vdash \alpha$.

Remark 6.49. The $\operatorname{card}\left(\operatorname{Hom}_{\mathcal{E}}(1, \Omega)\right)$ represents the number of truth-values in the multi-valued logic. For the examples SET ${ }^{n}$ and $\mathbf{S E T}^{n_{1}, n_{2}, \ldots, n_{s}}$ from 6.35 we get intuitionistic logic with $2^{n}$ truth values, and intuitionistic logic with $2^{n_{1}+n_{2}+\ldots+n_{s}}$ truth values, respectively. From the example 5). we get intuitionistic logic with 20 and, respectively, 6 truth values. From 6.36, 6)., it results that for any $n \in \mathbb{N}^{*}$, there is a topos $\mathcal{E}$ whose associated $I L$ has $n$ truth values.

In order to model theories $\mathcal{T}=(\mathcal{L}, \mathcal{A}, \mathcal{D})$ in topoi, we also need quantifiers, as remarked in section 5 at 5.52, 3. So, we now follow the ideas from 5.51, but in the Heyting algebra case.

Definition 6.50. A monadic Heyting algebra (abbreviated MHA) is an algebraic structure $(H,+, \cdot \rightarrow$ $, 0,1, \exists, \forall)$ where $(H,+, \cdot \rightarrow, 0,1)$ is a Heyting algebra (see 6.2), $\exists$ is a closure operator on $H$ (this 
means, it satisfies (i)-(iv) from 5.51), $\forall$ is an interior operator on $H$ (this means, it satisfies (i)'-(iv) ${ }^{\prime}$ from 5.51), and

(v) $\forall \exists a=\exists a$, for any $a \in H$;

(vi) $\exists \forall a=\forall a$, for any $a \in H$;

(vii) $\exists(\exists a \wedge b)=\exists a \wedge \exists b$, for any $a, b \in H$.

We end this section with

Example 6.51. Let $R \subset A \times B, A, B \in O b($ SET $)$ be any relation. Define then the quantifiers "along $R " \exists_{R}: \mathcal{P}(A) \rightarrow \mathcal{P}(B)$ and $\forall_{R}: \mathcal{P}(A) \rightarrow \mathcal{P}(B)$ by

$$
\begin{gathered}
\exists_{R}(X)=\{y \mid \exists x(x \in X \wedge x R y)\} \\
\forall_{R}(X)=\{y \mid \forall x(x R y \rightarrow x \in X)\} .
\end{gathered}
$$

If $A=B$, then $\exists_{R}, \forall_{R}: 2^{A}=\mathcal{P}(A) \rightarrow 2^{A}=\mathcal{P}(A)$, and $\left(2^{A}, \cup, \cap,-, \emptyset, A, \exists_{R}, \forall_{R}\right)$ becomes a MBA. If $A=B$ and $R="="$, then $\exists \forall$ are the usual quantifiers.

Definition 6.52. In a topos $\mathcal{E}$, let's consider a monomorphism $r: R \hookrightarrow a \times b, a, b \in O b(\mathcal{E})$. Then, in [Street and Brock] (topoi, page 457) actual arrows were constructed

$$
\begin{aligned}
& \forall_{r}: \Omega^{e} \rightarrow \Omega^{b} \\
& \exists_{r}: \Omega^{e} \rightarrow \Omega^{b}
\end{aligned}
$$

(where $\Omega$ is the subobjects classifier in $\mathcal{E}$, and $\Omega^{a}, \Omega^{b}$ are exponentiations), for which the previous $\forall_{R}$ and $\exists_{R}$ from SET are particular cases. If $a=b=1$, so $r \hookrightarrow 1 \times 1$, one gets

$$
\begin{aligned}
& \forall_{r}: \operatorname{Hom}_{\mathcal{E}}(1, \Omega) \rightarrow \operatorname{Hom}_{\mathcal{E}}(1, \Omega) \\
& \exists_{r}: \operatorname{Hom}_{\mathcal{E}}(1, \Omega) \rightarrow \operatorname{Hom}_{\mathcal{E}}(1, \Omega)
\end{aligned}
$$

Theorem 6.53. For any topos $\mathcal{E}$ the 8-uple $\left(\operatorname{Hom}_{\mathcal{E}}(1, \Omega), \wedge, \vee, \rightarrow, 0,1, \forall_{r}, \exists_{r}\right)$ is a MHA (the operations from the $\operatorname{Hom}_{\mathcal{E}}(1, \Omega)$ are those defined on the right-sides in 6.46). It is a MBA iff $\mathrm{E}$ is a Boolean topos.

\section{Summary on Non-standard Analysis in SET; Elements of Non-standard Analysis in SET-type Topoi}

Part of the material presented here follow [11], where most of the details can be found. We also mention the book [5]. However, none of the two books deal with Non-standard Analysis in topoi, our approach from 7.46 being original and giving openings to the case of the topoi of type $\mathrm{SET}^{\mathcal{C}}$ from Part II. In this paper we built non-standard analysis in topoi of the form $\mathbf{S E T}_{\overrightarrow{\mathcal{G}}}$, based on 5.34, 5 . 
and the following 7.46, ${ }^{*}$ 3and8.11,3.Weincludhere7.21, 7.29, 7.31asin[11]becousetheyholdin $\mathbf{S E T}^{\mathbf{n}} \overrightarrow{\mathcal{G}}$ as given in the quoted numbers, with appropriate (and natural) definitions (see $7.46,{ }^{*} 3$.).

In section 5 (at 5.10) we presented the $Z F$ axiomatic of the theory of sets. As we remarked at 5.12, the axiom 7 (Infinity) allows us to speak about the set of natural numbers, $\mathbf{N}$, where we can define an addition, as it follows:

$$
m, n \in \mathbf{N}, m+n:=\underbrace{S(S \ldots(S(m)) \ldots)}_{n \text { times }}
$$

where

$$
m=\underbrace{S(S \ldots(S(0)) \ldots)}_{m \text { times }}
$$

$(0=\emptyset$, the empty set, and $S(\omega)=\omega \cup\{\omega\}$, see 5.10, 7.). It is easy to check that

- $\forall m \forall n \forall p((m+n)+p=m+(n+p))$;

- $\forall n(n+0=0+n=n)$;

- $\forall m \forall n(m+n=n+m)$;

hold on $S$. This gives to $(\mathbf{N},+)$ the structure of commutative semigroup with neutral element.

Definition 7.1. We recall that a relative $\mathcal{R} \subseteq X \times X$ is called relation of equivalence if it is reflexive $((R): x \mathcal{R} x, \forall x \in X)$, symmetric $((S): x \mathcal{R} y \Rightarrow y \mathcal{R} x, \forall x, y \in X)$ and transitive $((T): x \mathcal{R} y \wedge y \mathcal{R} z \Rightarrow x \mathcal{R} z, \forall x, y, z \in X)$. The equality (of elements, of sets) and the congruence modulo $n \in \mathbf{N}^{*}\left(n \geq 2, \equiv_{n}\right)$ are two examples.

Definition 7.2. Having $(\mathbf{N},+)$ we can build the set $\mathbf{Z}$ of integers, using the equivalence relation $\mathcal{R}_{\mathbf{Z}}$ defined as it follows: $\mathcal{R}_{\mathbf{Z}} \subset \mathbf{N}^{2} \times \mathbf{N}^{2},(m, n) \mathcal{R}_{\mathbf{Z}}(k, l)$ iff $m+l=n+k$. The classes

$$
\left.\widehat{(k, l)}:=\left\{(m, n) \in \mathbf{N}^{2} \mid(m, n) \mathcal{R}_{\mathbf{Z}}(k, l)\right\}, k, l \in \mathbf{N}\right\}
$$

are called the equivalence classes of $\mathcal{R}_{\mathbf{Z}}$. Then $\mathbf{Z}:=\mathrm{N}^{2} / \mathcal{R}_{\mathbf{Z}}$, is the set of the equivalence classes.

One can define on $\mathbf{Z}$ the addition of classes, defined on components $\widehat{(k, l)}+\widehat{\left(k^{\prime}, l^{\prime}\right)}=\left(k+\widehat{k^{\prime}, l}+l^{\prime}\right)$, and we get a good definition (it does not depend on the representant $\left(k^{\prime}, l^{\prime}\right) \in \widehat{(k, l)}$ ). We get the structure $(\mathbf{Z},+)$ of commutative group (see 5.20,1. also). Here $\mathbf{N} \rightarrow \mathbf{Z}, n \mapsto \widehat{(n, 0)}$ is a monomorphism of semigroups, and $\mathbf{Z} \backslash \mathbf{N}=\{\widehat{(k, l)} \mid k<l\}$ are the negative integer numbers. We can also define on $\mathbf{N}$ a multiplication, as a repeated addition: $m \cdot n=\underbrace{n+n+\ldots+n}_{m \text { times }}$, which can be easily extended to $(\mathbf{Z},+, \cdot)$ such that it becomes an unitary commutative ring. In fact $\mathbf{N}$ has been extended to $\mathbf{Z}$ in order to be able to subtract any two elements. Now, in $(\mathbf{Z},+, \cdot)$ we also need to divide any two elements.

Definition 7.3. We need now the set $\mathrm{Q}$ of rational numbers. One proceeds as in 7.2 , but using the multiplication instead. Define $\mathcal{R}_{\mathbf{Q}} \subset\left(\mathbf{Z} \times \mathbf{Z}^{*}\right) \times\left(\mathbf{Z} \times \mathbf{Z}^{*}\right)$ by $(u, v) \mathcal{R}_{\mathbf{Q}}(s, t)$ iff $u \cdot t=v \cdot s\left(\mathbf{Z}^{*}=\mathbf{Z} \backslash\{0\}\right)$ $\mathcal{R}_{\mathbf{Q}}$ is a relation of equivalence and put: $\mathbf{Q}:=\mathbf{Z} \times \mathbf{Z}^{*} / \mathcal{R}_{\mathbf{Q}}$. Then $\frac{p}{q}:=\overline{(p, q)}$ (class). Then $(\mathbf{Q},+, \cdot)$ becomes a field with the operations (well defined):

$$
\frac{p}{q}+\frac{p^{\prime}}{q^{\prime}}=: \frac{p q^{\prime}+p^{\prime} q}{q q^{\prime}} ; \quad \frac{p}{q} \cdot \frac{p^{\prime}}{q^{\prime}}=: \frac{p p^{\prime}}{q q^{\prime}} .
$$


Moreover $\mathbf{Z} \rightarrow \mathbf{Q}, k \mapsto \frac{k}{1}$ is a monomorphisms of commutative unitary rings.

7.4. One also needs other numbers. For example if one considers a right triangle with both catheti equal to $1 \in \mathrm{N} \subset \mathrm{Q}$, by Pythagorean Theorem one gets the value denoted by $\sqrt{2}$ for the hypotenuse, which cannot be rational (use the fundamental theorem of arithmetic). Similarly, with other arguments, for $\pi=$ (length of a circle) / (its diameter). We need to complete $\mathrm{Q}$ to a bigger field, the field of real numbers denoted by $\mathbf{R}$.

Definition 7.5. On Q, one can define an order (a reflexive, antisymmetric, and transitive relation), compatible with the two algebraic operators, as it follows: $r=\frac{p}{q}>0$ iff either $p, q>0$, or $p, q<0$; $r=\frac{p}{q}<0$ iff either $p>0, q<0$ or $p<0, q>0 ; r=\frac{p}{q}=0$ iff $p=0 ; r_{1} \leq r_{2}$ iff $\left(r_{1}-r_{2}<0\right.$ or $r_{1}-r_{2}=0$ ); (see 7.3 and 7.2 for the definition of positive and negative integers).

Then $(\mathbf{Q},+, \cdot, \leq)$ becomes an ordered field.

Definition 7.6. The structure of order on $\mathrm{Q}$ allows us to define an absolute value $|\cdot|$ on $\mathrm{Q}$.

$$
|r|=\left\{\begin{aligned}
r, & \text { if } \quad r \geq 0 \\
-r, & \text { if } r<0
\end{aligned}\right.
$$

having the following fundamental properties:

$$
\begin{aligned}
& \left(N_{1}\right):|r| \geq 0, \forall r \in \mathbf{Q}, \text { and }|r|=0 \Leftrightarrow r=0 ; \\
& \left(N_{2}\right):|\lambda \cdot r|=|\lambda| \cdot|r|,(\forall) \lambda \in \mathbf{Q}, r \in \mathbf{Q} ; \\
& \left(N_{3}\right):\left|r_{1}+r_{2}\right| \leq\left|r_{1}\right|+\left|r_{2}\right|,(\forall) r_{1}, r_{2} \in \mathbf{Q} .
\end{aligned}
$$

7.7. Using the absolute value, one can define on $\mathrm{Q}$ two notions: the convergent sequences, and the Cauchy sequences.

Definition 7.8. If $M \in O b(\mathbf{S E T}), M \neq \emptyset$, we call a sequence of elements from $M$, any map $f: \mathbf{N} \rightarrow M$. We can write $f=\left(m_{n}\right)_{n \in \mathbf{N}}$, where $m_{n}=f(n) \in M$, for any $n \in \mathbf{N}$.

Definition 7.9. 1) A sequence of rational numbers $\left(r_{n}\right)_{n \in \mathbf{N}}, r_{n} \in \mathbf{Q}$ is called convergent iff $\exists r \in \mathbf{Q}$ such that $\forall m \in \mathbf{N}^{*} \exists N=N_{m}$ such that $\left|r_{n}-r\right|<\frac{1}{m} \forall n \geq N$.

2) A sequence of rational numbers $\left(r_{n}\right)_{n \in \mathbf{N}}, r_{n} \in \mathbf{Q}$ is called Cauchy sequence iff $\forall m \in \mathbf{N}$ $\exists N=N_{m}$ such that $\left|r_{p}-r_{q}\right|<\frac{1}{m} \forall p, q \geq \mathbf{N}$.

Remarks 7.10. 1) Any convergent sequence is Cauchy, but the converse implication is not necessary true.

2) Any sum and product (on components) of convergent (resp. Cauchy) sequences are convergent (resp. Cauchy).

Construction 7.11. Let $\mathcal{C}=(\{f: \mathbf{N} \rightarrow \mathbf{Q} \mid f$ Cauchy $\},+, \cdot)$ (see 7.10.2). $\mathcal{C}$ becomes a commutative unitary ring. Let now $J=\{f: \mathbf{N} \rightarrow \mathbf{Q} \mid f$ converges to 0 (zero) $\}$. Then: $f, g \in J \Rightarrow f+g \in J$ and 
$\lambda \in \mathcal{C}, f \in J \Rightarrow \lambda \cdot f \in J$ (we say that $J$ is an ideal in $\mathcal{C}$ ). Then consider the relation $\mathcal{R}_{\mathbf{R}} \subset \mathcal{C} \times \mathcal{C}$, $\lambda \mathcal{R}_{\mathbf{R}} \mu \stackrel{\text { def }}{\Leftrightarrow} \lambda-\mu \in J$, which is a relation of equivalence, compatible with the operations from $\mathcal{C}$. Then put $\mathbf{R}:=\mathcal{C} / \mathcal{R}_{\mathbf{R}}$. The + and $\cdot$ from $\mathcal{C}$ factorise on $\mathbf{R}$. If we put $\lambda=\left(r_{n}\right)_{n} \mu=\left(s_{n}\right)_{n}, \lambda, \mu \in \mathcal{C}, \lambda<\mu$ iff $r_{n}<s_{n}$ for $n>>0$, and $\lambda \leq \mu$ iff $\lambda-\mu \in J$ or $\lambda<\mu$, we get on $\mathbf{R}$ an order compatible with the induced operations:

$\lambda \leq \mu \Rightarrow \lambda+\alpha \leq \mu+\alpha, \forall \lambda, \mu, \alpha \in \mathbf{R}$

$\lambda \leq \mu \wedge \beta \geq 0 \Rightarrow \lambda \cdot \beta \leq \mu \cdot \beta, \forall \lambda, \mu, \beta \in \mathbf{R}$.

Then $(\mathbf{R},+, \cdot, 0,1, \leq)$ becomes an (total) ordered field. Moreover, in $\mathbf{R}$ any Cauchy sequence is convergent, so $\mathbf{R}$ is a complete totally ordered field. Any two such objects are uniquely isomorphic, so $\mathbf{R}$ is unique in this sense. We call it the field of real numbers (another construction of the field of real numbers uses the so-called Dedekind cuts). It can be easily proved that $\mathbf{R}$ is an Archimedean field (this means that the subset of integers is not upperbounded).

Remark 7.12. The construction 7.11 shows us that two Cauchy sequences from $Q$ are identified in $\mathbf{R}$ iff they have the same limit, so $\mathbf{R}$ adds to $\mathbf{Q}$ all the limits of rational Cauchy sequences which are not already in $\mathbf{Q}$ (the inclusion $\mathbf{Q} \subset \mathbf{R}$ is given by the constant sequences). The limit in $\mathbf{R}$ is unique, so, a sequence of rational numbers converging in $\mathbf{R}$ to $\sqrt{2} \notin \mathbf{Q}$ is Cauchy (being convergent in $\mathbf{R}$ it is Cauchy in $\mathbf{R}$, so also in $\mathbf{Q}$, being a sequence of rational numbers), but not convegent in $\mathbf{Q}$ (since its unique limit does not belong to $\mathrm{Q}$ ). This shows us that $\mathrm{Q}$ is not complete.

Remark 7.13. Heuristically, we can write $\mathbf{R}=\left\{a_{1} a_{2} \ldots a_{s}, b_{1} b_{2} \ldots b_{t} \ldots \mid s \in \mathbf{N}, a_{1}, \ldots, a_{s}\right.$ are digits, and the $\left(b_{t}\right)_{t \in \mathbf{N}^{*}}$ 's are sequences of digits (in any fixed numeration base) $\}$.

If $x \in \mathbf{R}$, then $x \in \mathbf{Q}$ iff it is a periodic (simple or mixed) decimal fraction.

Construction 7.14. The equation $X^{2}=1$ has two real roots $( \pm 1)$, but the equation $X^{2}=-1$ has none. In order to fix this anomaly one has to further extend $\mathbf{R}$ to $\mathbf{C}$, the field of complex numbers. Let $\mathbf{R}[X]$ the ring of polynomials with real coefficients, and $I=\left\{f \cdot\left(X^{2}+1\right) \mid f \in \mathbf{R}[X]\right\}$. Then $I$ is an ideal in $\mathbf{R}[X]$ (see 7.11.) Put then $\mathbf{C}:=\frac{\mathbf{R}[X]}{\left(X^{2}+1\right)}$ (that is $\mathbf{R}[X] / \mathcal{R}_{\mathbf{C}}$, where $\mathcal{R}_{\mathbf{C}}$ is the equivalence relation given by $P \mathcal{R}_{\mathbf{C}} Q$ iff $P-Q \in I$ ). This factorization is compatible with the usual addition and multiplication of polynomials, conferring $\mathbf{C}$ a structure of algebraically closed field, see 7.17 (this means that any polynomial equation of degree $n$ has at least one complex root (so, exactly $n$, counted with multiplicities; in particular the previous equation $X^{2}=-1$ acquires exactly 2 roots in $\mathrm{C}$ )). This fact is sometimes called The Fundamental Theorem of Algebra.

Remark 7.15. Starting from the empty set and the axiomatic of $\mathrm{ZFC}$, we built $\mathrm{N} \subset \mathrm{Z} \subset \mathrm{Q} \subset$ $\mathbf{R} \subset \mathbf{C}$. Certainly, there are many other more complicated objects which could be further built, using functions, relations, relations between functions, functions of relations, function of functions of ... relations of ... relations, ... In such a way one develops mathematics using only the $\mathrm{ZF}(\mathrm{C})$-frame (axioms, which, in particular, postulate the existence of the empty set), see 5.10 and 5.16. 
Definition 7.16. The Theory of really closed fields is the theory $\mathcal{T}_{r c}=\left(\mathcal{L}, \mathcal{A}_{r c}, \mathcal{D}\right)$, with $\mathcal{L}$ as usual, $\mathcal{D}=\{M P, G, P, \ldots\}$ (see section 5), and $\mathcal{A}_{r c}$ is the set of axioms defining (canonically) the theory of totally ordered fields, plus two more axioms:

$$
\begin{aligned}
& \mathcal{A}_{1}: \forall x \exists y\left(x \geq 0 \wedge y^{2}=x\right) \\
& \mathcal{A}_{2}: \forall n \forall y_{0} \forall y_{1} \forall \ldots \forall y_{2 n+1} \exists x\left[\neg\left(y_{2 n+1}=0\right) \wedge\left(\mathrm{y}_{2 n+1} x^{2 n+1}+y_{2 n} x^{2 n}+\ldots+y_{1} x_{1}+y_{0}=0\right)\right] \\
& (\mathbf{R},+, \cdot, 0,1, \leq) \text { (the field of real numbers) is a model of the previous theory. }
\end{aligned}
$$

Definition 7.17. The Theory of algebraically closed fields is the theory $\mathcal{T}_{a c}=\left(\mathcal{L}, \mathcal{A}_{a c}, \mathcal{D}\right)$, with $\mathcal{L}$ and $\mathcal{D}$ as before, and $\mathcal{A}_{a c}$ is the set of axioms defining the theory of fields, plus the axiom

$\mathcal{A}_{3}: \forall n \forall y_{0} \forall y_{1} \forall \ldots \forall y_{n} \exists x\left[\neg\left(y_{n}=0\right) \wedge\left(y_{n} x^{n}+\ldots+y_{n-1} x^{n-1}+\ldots+y_{1} x_{1}+y_{0}=0\right)\right]$.

$(\mathbf{C},+, \cdot, 0,1)$ (the field of complex numbers) is a model of this theory (by the fundamental Theorem of algebra).

Definition 7.18. 1) If $\mathcal{U}=\mathcal{U}(T), T \neq \emptyset$ (see 5.16) one says that $\mathcal{L}_{0}$ is a first order language on $\mathcal{U}$ if one allows quantifications only after the atoms, which are the elements of $T$; an atom $A \in T$ is requested to satisfy the axiom: $\forall x(x \in \mathcal{P}(A) \rightarrow x=\emptyset \vee x=A)$.

2) If $\mathcal{U}$ is as before (but now possibly $T=\emptyset$ ) one says that $\mathcal{L}$ is a higher-order language if one allows to quantify over all the elements of $\mathcal{U}$ (atoms, functions, relations, ...).

Theorem 7.19. 1) If $\mathcal{T}_{r c}$ has the (first order) language $\mathcal{L}_{0}$, then it is model complete (that is, for any two models $R_{1}$ and $R_{2}$ of $\mathcal{T}_{r c}$, and sentence $\varphi$, we have $R_{1} \leq R_{2} \Rightarrow R_{1} \models \varphi$ iff $R_{2} \models \varphi$ ).

2). If $\mathcal{T}_{a c}$ has the (first order) language $\mathcal{L}_{0}$, then it is $\aleph_{0}-$ categorical (that is, for any two models $C_{1}$ and $C_{2}$ of $\mathcal{T}_{a c}$ with cardinal $>\aleph_{0}$, and sentence $\varphi$, we have $C_{1} \models \varphi$ iff $C_{2} \models \varphi$; in particular, any two models with cardinality $>\aleph_{0}$ are isomorphic iff they have the same cardinal).

We pass now to an enlargement of the standard universe $\mathcal{U}$ (with its mathematics), $\mathcal{U} \subset{ }^{*} \mathcal{U}$, called the Non-standard Enlargement, introduced by Abraham Robinson in [Ro]. But before this we need a few preliminaries.

7.20. Let's denote by $\mathcal{U}$ an Universe which is a family of sets satisfying some axioms. Of course, the governing ones (implicitly assumed) will be $Z F C$ and $C L$ from section 5. Recall that we presumed the existence of two predicates " $\in$ " and "=", used in ZFC. If $A$ is a set, then $\mathcal{P}(A)$ is the set of parts of $A$ (by $Z F C$ ). The axioms for $\mathcal{U}$ are:

\section{(ST) Strong Transitivity}

$\forall A \in \mathcal{U} \exists B \in \mathcal{U} B$ transitive with $A \subseteq B \subseteq U$ ( $B$ is called transitive if $b \in B \in \mathcal{U} \rightarrow b \in \mathcal{U}$ )

(P) Pairing

$\forall a \forall b(a, b \in \mathcal{U} \rightarrow\{a, b\} \in \mathcal{U})$

(U) Union

$\forall A \forall B(A, B \in \mathcal{U} \rightarrow A \cup B \in \mathcal{U})$

(PS) Power set 


$$
\forall A(A \in \mathcal{U} \rightarrow \mathcal{P}(A) \in \mathcal{U})
$$

A universe is called Universe over $X$ (if $X$ is a set, $X \in \mathcal{U}$ ), and the elements of $X$ are called individuals, satisfying: $(\forall x \in X)(x \neq \emptyset \wedge(\forall y \in \mathcal{U}) y \notin x)$ (the set of atoms of $\mathcal{U}$ ), compare with 7.18,1.

7.21 Properties of $\mathcal{U}$. From the previous axioms it can be proved that:

- $A \in \mathcal{U} \rightarrow\{a\} \in \mathcal{U}$;

- $A_{1}, \ldots, A_{m} \in \mathcal{U} \rightarrow A_{1} \cup \ldots \cup A_{m} \in \mathcal{U}$;

- $\mathcal{U}$ contains all its finite subsets: if $A \subset \mathcal{U}$ is finite, then $A \in \mathcal{U}$;

- $A \subseteq B \in \mathcal{U} \rightarrow A \in \mathcal{U}$;

- $\emptyset \in \mathcal{U}$;

- $\left\{A_{i} \mid i \in I\right\} \subseteq A \in \mathcal{U} \rightarrow \bigcup_{i \in I} A_{i} \in \mathcal{U}$ (this uses the strong transitivity);

- $\mathcal{U}$ is closed under unions of sets of sets;

- $\mathcal{U}$ is closed under arbitrary intersections;

- $a, b \in \mathcal{U} \rightarrow(a, b):=\{a,\{a, b\}\} \in \mathcal{U}$

- $A, B \in \mathcal{U} \wedge R \subseteq A \times B \rightarrow R \in \mathcal{U}$;

- $A_{1}, \ldots, A_{m} \in \mathcal{U} \wedge R \subseteq A_{1} \times A_{2} \times \ldots \times A_{m} \rightarrow R \in \mathcal{U}$;

- If $R \in \mathcal{U}$ is a binary relation, then $\operatorname{dom} R, \operatorname{ran} R, R$-image $R^{i}(C)$ of any $C \subseteq \operatorname{dom} R, R^{-1}$ all $\in \mathcal{U}$, where

$$
\begin{aligned}
& \operatorname{dom} R=:\{a \mid \exists b((a, b) \in R)\}, \\
& \operatorname{ran} R=:\{b \mid \exists a((a, b) \in R)\}, \\
& R^{i}(C)=:\{b \mid \exists a \in C((a, b) \in R\}, \\
& R^{-1}=:\{(b, a) \mid(a, b) \in R\} ;
\end{aligned}
$$

- $R, S \in \mathcal{U}$ binary relations, then $R \circ S \in \mathcal{U}$, where $R \circ S=:\{(a, c) \mid \exists b((a, b) \in R \wedge(b, c) \in S)\}$;

- If $f: A \rightarrow B$ is a function with $A, B \in \mathcal{U}$, then $f \in \mathcal{U}$; moreover, for any $C \subseteq A$ and $D \subseteq B$ $f^{i}(C) \in \mathcal{U}, f^{-1}(D) \in \mathcal{U}$, where $f^{i}(C)=:\{f(a) \mid a \in C\}, f^{-1}(D)=:\{a \in A \mid f(a) \in D\}$;

- $A, B \in \mathcal{U} \rightarrow B^{A} \in \mathcal{U}$;

- $\left(\left\{A_{i} \mid i \in I\right\} \in \mathcal{U} \wedge I \in \mathcal{U}\right) \rightarrow\left(\prod_{i \in I} A_{i}\right) \in \mathcal{U}$.

Example 7.22. If $T$ is a set (possibly the empty set), then $\mathcal{U}=\mathcal{U}(T)$ is an Universe (see 5.16) over $T$.

Example 7.23. The triplet $\left(\mathcal{L}_{\mathcal{U}}, \mathcal{A}, \mathcal{D}\right)$, with $\mathcal{L}_{\mathcal{U}}$ as in $5.17, \mathcal{A}$ consists of axioms 7.20 , and $\mathcal{D}=\{M P, G, P\}(5.7)$, represents the Theory of Universes, having the $\mathcal{U}=\mathcal{U}(T)$ 's as models $(\mathcal{U}(T)$ is the smallest universe containing $T$ ).

Example 7.24. The biggest $\mathcal{U}$ is $O b(S E T)$, the class of all the sets. $\mathcal{U}$ is not a set, but when doing mathematics we are dealing with elements of $\mathcal{U}$, which are sets.

7.25. The Non-standard extensions. Let $\mathcal{U}=\mathcal{U}(T) \stackrel{*}{\hookrightarrow} \mathcal{U}^{\prime}$ a monomorphism (injective map) of universes.Let's denote by ${ }^{*} \mathcal{U}:=*(\mathcal{U}) \subseteq \mathcal{U}^{\prime}$ and by ${ }^{*} a$ the image of $a,{ }^{*} a:=*(a), \forall a \in \mathcal{U}$. For a 
sentence $\varphi$ in $\mathcal{L}_{\mathcal{U}}$, let's denote by ${ }^{*} \varphi$ the sentence in $\mathcal{L}_{\mathcal{U}^{\prime}}$ obtained by keeping unchanged the logical quantifiers $\vee, \wedge, \neg, \rightarrow, \forall, \exists$ and replacing all the constant symbols $a \in \mathcal{U}$ by ${ }^{*} a \in \mathcal{U}^{\prime}$. One requests that $*$ satisfies the axioms:

- ${ }^{*} a=a, \forall a \in T$ (if $\mathcal{U}=\mathcal{U}(T)$, for $T=\emptyset$ this condition doesn't exist);

- $\emptyset=\emptyset$;

- $S, T$ : An $\mathcal{L}_{\mathcal{U}}$ - sentence $\varphi$ is true $\Leftrightarrow{ }^{*} \varphi$ is true as $\mathcal{L}_{\mathcal{U}^{\prime}}$ - sentence; the $\Rightarrow$ is called transfer $(\mathbf{T})$, and $\Leftarrow$ is called standardization $(\mathbf{S})$;

- I (idealisation): If $\mathcal{R} \in \mathcal{U}$ is any finitely satisfiable binary relation (i.e. $\forall n \forall x_{1}, \ldots, x_{n} \exists y\left(y R x_{1} \wedge\right.$ $\left.\ldots \wedge y R x_{n}\right)$ ), then ${ }^{*} \mathcal{R}$ is globally satisfiable (i.e. $\exists y^{\prime} \forall x\left(y^{*} \mathcal{R} x\right)$ ). We should remark that the $x$ 's and $y$ run inside $\mathcal{U}$, while $y^{\prime}$ runs through $\mathcal{U}^{\prime}\left({ }^{*} x=x, \forall x \in \operatorname{dom} \mathcal{R}, y \in \mathcal{U}, y^{\prime} \in \mathcal{U}^{\prime}\right)$. We note that the order of the variables is not important, because, if $\mathcal{R}=\mathcal{R}(x, y)$ is a binary relation, so is $\mathcal{R}(y, x)=\mathcal{R}^{-1}$.

Remark 7.26. 1. If $\mathcal{U}$ is a general Universe, then, in the conditions from 7.25 , we restrict the monomorphism * at $\mathcal{U}(T) \subset \mathcal{U}$, for $T=\left\{\left.a \in \mathcal{U}\right|^{*} a=a\right\}$. The triplet $\left(\mathcal{L}=\left(\mathcal{L}_{\mathcal{U}}, \mathcal{L}_{\mathcal{U}^{\prime}}\right), Z F C+I+S+T\right.$, $\mathcal{D}=\{M P, G, P\})$ represents the Theory of non-standard extensions. We point out that this theory has a higher order logic, see 7.18, 2.

2. We call $\mathrm{ZFC}+\mathrm{CL}+\mathrm{I}+\mathrm{S}+\mathrm{T}=\mathrm{IST}$ (internal set theory). If $\mathcal{U}$ has ZFC then $* \mathcal{U}$ has IST as axiomatics. Moreover, a sentence $\varphi$ in $\mathcal{L}_{\mathcal{U}}$ conserves its truth value $(0$ or 1$)$ when passing to ${ }^{*} \varphi$ in ${ }^{*} \mathcal{U}$. We say that the extension $\mathcal{U} \stackrel{*}{\hookrightarrow} * \mathcal{U}$ is conservative. Moreover, $\mathcal{U}$ is not contradictory iff $* \mathcal{U}$ is (because we can construct models for ${ }^{\mathcal{U}}$ starting from $\mathcal{U}$ with $Z F C$, see 7.38).

So ${ }^{*} \mathcal{U}$ has a $\mathrm{CL}$ and $\mathcal{U} \subset{ }^{*} \mathcal{U}$ is conservative. We'll get later examples in Topoi, for IL (for topoi of SET-type in this section; for topoi of the exponential type $\mathbf{E N S}^{\mathcal{C}}$ see part II.Artificial Intelligence, section 3 , see 5.35 also).

Theorem 7.27. For any infinite set $T$ there exists an enlargement of $\mathcal{U}(T)$ that is of the form $\mathcal{U}\left({ }^{*} T\right)$.

So ${ }^{*} \mathcal{U}$ has a $\mathrm{CL}$ and $\mathcal{U} \subset{ }^{*} \mathcal{U}$ is conservative.

7.28 Examples/Definitions. 1. Apply the axiom idealization (I) to the binary relation $>$ (inequality), $>\subset \mathbf{R} \times \mathbf{R}$ ( $\mathbf{R}$ is the field of real numbers). This relation is finitely satisfiable in $\mathrm{x}$, so we get an $y^{\prime} \in{ }^{*} \mathbf{R}$ such that $y^{\prime}>x, \forall x \in \mathbf{R}$. Such (non-standard) $y^{\prime}$ 's are called unlimited real numbers.

2. Apply the same axiom (I) to the binary relation $<\subset(0, \infty) \times(0, \infty)$, which is the usual inequality of real numbers. The relation $<$ is finitely satisfiable in the second variable (for any $\epsilon_{1}, \ldots, \epsilon_{n}>0$, there is $x>0$, such that $\left.x<\epsilon_{1}, \ldots, x<\epsilon_{n}\right)$ so, we get an $x^{\prime} \in{ }^{*}(0, \infty)$ s.t. $x^{\prime}<\epsilon$, for any $\epsilon>0$. Passing from $(0, \infty)$ to $\mathbf{R}$ (replacing the previous positive $x$ with $|x|$ for any real $x$, we get the existence of elements $x \in{ }^{*} \mathbf{R}$ with the property that $x \neq 0$ and $|x|<\epsilon$, for any $\epsilon>0$. Such (non-standard) $x$ 's are called infinitesimals. Moreover, we call the halo of zero, the set $h a l(0):=\left\{x \in{ }^{*} \mathbf{R} \mid x\right.$ infinitesimal $\}$ $\cup\{0\}$ and, for any $a \in \mathbf{R}$ we put $h a l(a):=\left\{b \in{ }^{*} \mathbf{R} \mid b-a \in \operatorname{hal}(0)\right.$, both positive and negative $\}$.

3. Now apply the axiom (I) to the binary relation $\mid \subset \mathbf{N} \times \mathbf{N}$ defined by $a \mid b$ if a divides $b$. We get the existence of an $b^{\prime} \in{ }^{*} \mathbf{N}$ such that $a \mid b^{\prime}$ for any $a \in \mathbf{N}$. Similarly, for an arbitrary prime number $p \in \mathbf{N}$ we get the existence of an $\omega \in{ }^{*} \mathbf{N}$ such that $p^{n} \mid p^{\omega}$ for any $n \in \mathbf{N}$. 
4. Let's apply now the axiom of trasfer (T) to the fundamental theorem of arithmetic, which says that any natural number can be uniquely written as a finite product of powers of distinct prime numbers (modulo the order of the factors). We get : $\forall \omega \in{ }^{*} \mathbf{N} \exists \eta \in{ }^{*} \mathbf{N} \exists \nu_{1}, \ldots, \nu_{\eta} \in{ }^{*} \mathbf{N} \exists P_{1}, \ldots P_{\eta} \in{ }^{*} \mathbf{N}$ prime (hiperfinite) numbers with $\omega=P_{1}^{\nu_{1}} P_{2}^{\nu_{2}} \ldots P_{\eta}^{\nu_{\eta}}\left(P \in{ }^{*} \mathbf{N}\right.$ is prime if $\forall a \in{ }^{*} \mathbf{N} a \mid P \Rightarrow a=$ $1 \vee a=P$ ). So, the fundamental theorem of arithmetic in ${ }^{*} \mathbf{N}$ says that any non-standard natural number can be uniquely written as a hyperfinite product of hyperfinite powers of hyperfinite prime numbers (modulo the order of the factors). We note here that the hyperfinite sums $\left(k_{1}+k_{2}+\ldots+k_{\omega}, \omega \in{ }^{*} \mathbf{N}\right)$ and hyperfinite products $\left(k_{1} k_{2} \ldots k_{\omega}, \omega \in{ }^{*} \mathrm{~N}\right)$ are also obtained using the axiom (T), from the usual finite sums and products.

\subsection{Properties of *}

- $a=b$ iff ${ }^{*} a={ }^{*} b$ so $a \mapsto{ }^{*} a$ is injective;

- $a \in B$ iff * $a \in *^{*} B$

- $A \subseteq B$ iff * $A \subset{ }^{*} B$;

- If $A \subset T$ then $A \subseteq{ }^{*} A \subseteq{ }^{*} T$; in particular $T \subset{ }^{*} T$;

- $(A \cap B)={ }^{*} A \cap{ }^{*} B$;

- $(A \cup B)={ }^{*} A \cup{ }^{*} B$;

- $(A \backslash B)={ }^{*} A \backslash{ }^{*} B$

- ${ }^{*} A=A$ iff $A$ is finite; otherwise * $A \backslash A \neq \emptyset$;

- $A$ transitive set in $\mathcal{U} \Rightarrow{ }^{*} A$ transitive in ${ }^{*} \mathcal{U}$;

- ${ }^{*} \mathcal{P}(A) \subseteq \mathcal{P}\left({ }^{*} A\right)$;

- $\left(a_{1}, \ldots, a_{m}\right)=\left({ }^{*} a_{1}, \ldots,{ }^{*} a_{m}\right), \forall m$;

- $\left(a_{1}, \ldots, a_{m}\right) \in R$ iff $\left({ }^{*} a_{1}, \ldots,{ }^{*} a_{m}\right) \in{ }^{*} R$;

- $\left(A_{1} \times \ldots \times A_{m}\right)={ }^{*} A_{1} \times \ldots \times{ }^{*} A_{m}\left({ }^{*}\left(A^{m}\right)=\left({ }^{*} A\right)^{m}\right)$;

- If $R \in \mathcal{U}$ is a binary relation, then:

* $(\operatorname{dom} R)=$ dom $^{*} R$;

${ }^{*}(\operatorname{ran} R)=\operatorname{ran}^{*} R$

${ }^{*}\left(R^{-1}\right)=\left({ }^{*} R\right)^{-1}$

${ }^{*}\left(R^{i}(C)\right)=\left({ }^{*} R\right)^{i}\left({ }^{*} C\right), C \subseteq \operatorname{dom} R$;

${ }^{*}\left(R^{-1}(D)\right)=\left({ }^{*} R\right)^{-1}\left({ }^{*} D\right), D \subseteq \operatorname{ran} R$.

(see 7.21 for definitions).

- if $R, S$ are binary relations $\subset \mathcal{U}$, then ${ }^{*}(R \circ S)=\left({ }^{*} R\right) \circ\left({ }^{*} S\right)$;

- if $f: A \rightarrow B$ is a function, $f \in \mathcal{U}$, then ${ }^{*} f:{ }^{*} A \rightarrow{ }^{*} B$ with ${ }^{*}(f(a))={ }^{*}\left(f\left({ }^{*} a\right)\right), \forall a \in A$, and ${ }^{*} f \in{ }^{*} \mathcal{U}$; moreover $f$ injective, surjective, bijective iff ${ }^{*} f$ is so

Definition 7.30. A set $B \in \mathcal{U}^{\prime}$ is called standard if $\exists A \in \mathcal{U}$ with $B={ }^{*} A$, otherwise it is called non-standard. A non-standard set $B \in{ }^{*} \mathcal{U}$ is called internal if $\exists A \in \mathcal{U}$ with $B \in{ }^{*} A$ (we use both primary relations "=" and " $\exists ")$ and external otherwise.

\subsection{Properties of internal sets}

- $A, B$ internal $\Rightarrow A \cap B, A \cup B, A \backslash B, A \times B$ internal; 
- the union and intersection of any internal collection of sets are internal: if $\left\{A_{i} \mid i \in I\right\} \in{ }^{*} \mathcal{U}$ and $A_{i}$ internal for any $i$, then $\bigcup_{i \in I} A_{i}$ and $\bigcap_{i \in I} A_{i}$ are internal;

- if $a_{1}, \ldots, a_{m}$ are internal, then both $\left\{a_{1}, \ldots, a_{m}\right\}$ and $\left(a_{1}, \ldots, a_{m}\right)$ are internal;

- if $R$ is an internal binary relation, then dom $R$, ram $R, R^{-1}, R^{i}(C),(C \subseteq$ dom $R$ internal) are internal $\left(R={ }^{*} R\right)$;

- $R, S$ binary internal relations so is $R \circ S$;

- if $f: A \rightarrow B$ is internal, $a \in \operatorname{dom} f=A$ internal, then $f(a)$ is internal; $C \subseteq A$ internal, then $f^{i}(C)$ is internal; $D \subset B$ internal, then $f^{-1}(D)$ is internal.

- ${ }^{*} \mathcal{U}$ is the set of internal sets, and ${ }^{*} \mathcal{P}(A)=$ set of internal subsets of ${ }^{*} A$, that is ${ }^{*} \mathcal{P}(A)=\mathcal{P}\left({ }^{*} A\right) \cap{ }^{*} \mathcal{U}=\{B \subseteq * A / B$ internal $\}$.

\section{Example 7.32.}

1. $\mathbf{N}$ is an external set in $\mathcal{U}^{\prime}$.

2. ${ }^{*}(A):=\left\{{ }^{*} a \mid a \in A\right\}, A$ infinite is external (note that ${ }^{*}(A) \neq{ }^{*} A$ ).

Theorem 7.33. For $\mathcal{U} \stackrel{*}{\hookrightarrow} \mathcal{U}^{\prime}$ as before, let $\varphi(x)$ be an internal $\mathcal{L}_{\mathcal{U}^{\prime}}$-formula (i.e. all its constants are internal) having $x$ as the only free variable. Then, for any internal set $A$, the set $\{a \in A \mid \varphi(a)$ is true $\}$ is internal.

Corollary 7.34. Let $f: A \rightarrow B$ function, with $A$ and $B$ internal sets. Let's suppose that there is an internal $\mathcal{L}_{\mathcal{U}^{\prime}}$ - term (see 5.3) $\tau(x)$ with $x$ the only variable such that $f(a)=\tau(a), \forall a \in A$. Then $f$ is an internal function.

Definition 7.35. Let's denote by $\mathcal{P}_{F}(A)=\{B \subseteq A \mid B$ finite $\}$. Then $\mathcal{P}_{F}(A) \in \mathcal{U}$. Let's consider the $\mathcal{L}_{\mathcal{U}}$-sentence

$$
\varphi:(\forall n \in \mathbf{N})\left(\exists V \in \mathcal{P}_{F}(\mathbf{N})\right)(\forall x \in \mathbf{N})[x \in V \rightarrow x \leq n] .
$$

$\varphi$ is true and asserts that $\{1,2, \ldots n\} \in \mathcal{P}_{F}(\mathbf{N})$ for any $n \in \mathbf{N}$. But $\varphi$ true $\Leftrightarrow^{*} \varphi$ true $(S+T)$, so for any $n \in{ }^{*} \mathbf{N}$, the initial segment

$$
\{1, \ldots, n\}=\left\{x \in{ }^{*} \mathbf{N} \mid x \leq n\right\}
$$

of ${ }^{*} \mathbf{N}$ belongs to ${ }^{*} \mathcal{P}_{F}(\mathbf{N})$. Then, the members of ${ }^{*} \mathcal{P}_{F}(\mathbf{N})$ are the transferred notions of the finite members of $\mathbf{N}$. We say that a set $B \in \mathcal{U}^{\prime}$ is hiperfinite iff it belongs to ${ }^{*} \mathcal{P}_{F}(A)$ for some $A \in \mathcal{U}$. Any number $\omega \in^{*} \mathbf{N} \backslash \mathbf{N}$ is called hyperfinite.

Theorem 7.36. A set $B$ is hyperfinite iff there is an internal bijection $f:\{1,2, \ldots, n\} \rightarrow B$ for some $n \in{ }^{*} \mathbf{N}$ (note that when $n \in \mathbf{N} \subset{ }^{*} \mathbf{N}$, the set $B$ is finite, in the usual sense).

The previous statements and definitions were presented in the frame of the theory of the Theory of non-standard sets, IST (note that we do not get here exactly the same theory from [Nelson], but a similar one). We continue now by indicating a concrete model of the nonstandard Univers (extension, 
enlargement), starting with a standard one with the axioms from $C L$ and $Z F C$.

Theorem 7.37; Some Permanence Principles. Let $\phi(x)$ be an internal $\mathcal{L}_{\mathcal{U}}$-formula (see 7.33) with $\mathrm{x}$ the only free variable. Then :

1).(Overflow) If there is $k \in \mathbf{N}$ such that $\phi(n)$ is true for all $n \in \mathbf{N}$ with $n \geq k$, then there exists $K \in{ }^{*} \mathbf{N} \backslash \mathbf{N}$ such that $\phi(n)$ is true for all $n \in{ }^{*} \mathbf{N}$ with $k \leq n \leq K$.

2).(Underflow) If there is $K \in{ }^{*} \mathbf{N} \backslash \mathbf{N}$ such that $\phi(n)$ is true for all $n \in{ }^{*} \mathbf{N} \backslash \mathbf{N}$ with $n \leq K$ then there exists $k \in \mathbf{N}$ such that $\phi(n)$ is true for all $n \in \mathbf{N}$ witk $k \leq n \leq K$.

3). If $\phi(a)$ is true on a neighborhood of some $b \in \mathbf{R}$ contained in hal $(b)$ - see 7.28, 2. (for instance on the whole $h a l(b))$, then $\phi(a)$ is true for all $a$ within some real positive distance from $b$.

4). If $\phi(a)$ is true for arbitrarily large (resp. small) $a \in \mathbf{R}$, then it is true for some positive (resp. negative) unlimited $b \in{ }^{*} \mathbf{R}$ (see $7.28,1$.).

5). If there is $r \in \mathbf{R}$ such that $\phi(a)$ is true for all $a \in \mathbf{R}$ with $a \geq r$, then there is a positive unlimited $b \in{ }^{*} \mathbf{R}$ such that $\phi(a)$ is true for all $a \in{ }^{*} \mathbf{R}$ with $r \leq a \leq b$.

Definition 7.38. Let $I \neq \emptyset$ be a set. A subset $\mathcal{F}_{0} \subset \mathcal{P}(I)$ is called a filter on $I$ if it satisfies :

$\left(F_{1}\right) \emptyset \notin \mathcal{F}_{0}, I \in \mathcal{F}_{0}$

$\left(F_{2}\right) A, B \in \mathcal{F}_{0} \Rightarrow A \cup B \in \mathcal{F}_{0}$;

$\left(F_{3}\right) A \in \mathcal{F}_{0}$ and $A \subset B(\subset I) \Rightarrow B \in \mathcal{F}_{0}$.

$\mathcal{F} \subset \mathcal{P}(I)$ is called an ultrafilter if it satisfies $\left(F_{1}\right),\left(F_{2}\right),\left(F_{3}\right)$ and

$\left(F_{4}\right) \forall A \in \mathcal{P}(I)\left[(A \in \mathcal{F}) \vee\left(C_{I} A \in \mathcal{F}\right)\right]$.

Let $a \in I$ an arbitrary element. Then the family $\mathcal{F}_{a}=\{A \in \mathcal{P}(I) \mid a \in A\}$ is an ultrafilter. Such kind of ultrafilters are called principal ultrafilters (generated by one element). An ultrafilter is called nonprincipal if it is not of the previous form.

Remarks 7.39. 1. $\mathcal{F}_{0}$ can be taken as being $\{J \in \mathcal{P}(I) \mid I \backslash J$ is finite $\}$.

2. Let $\mathcal{A}:=(\{\mathcal{F} \mid \mathcal{F}$ filter on I $\}, \subseteq)$. Then it is easy to see that $\mathcal{A}$ is inductively ordered (this means that any totally ordered subfamily of A has a majorant); so, by Zorn' s Lemma (see 5.15) $\mathcal{A}$ has a maximal element $\mathcal{F}$. It can be shown that this $\mathcal{F}$ is an ultrafilter. Doing the same thing with the filters greater than a fixed one $\left(\mathcal{F}_{0}\right.$ say) we deduce that any filter on I can be extended to an ultrafilter on I.

Definition-Theorem 7.40. 1 . Let I be an infinite set, $\left(X_{i}\right)_{i \in I}$ a family of sets, and $\mathcal{F}$ a nonprincipal ultrafilter on I (see 7.38). We call the ultraproduct of the $X_{i}$ 's after the ultrafilter $\mathcal{F}$, the quotient set

$\mathfrak{U}_{i \in I} X_{i}:=\left(\prod_{i \in I} X_{i}\right) / \mathcal{F}$

where the factorization by $\mathcal{F}$ means that we factorized by the equivalence relation $\mathcal{R}_{\mathcal{F}}$ defined by $\left(x_{i}\right)_{i \in I} \mathcal{R}_{\mathcal{F}}\left(y_{i}\right)_{i \in I}$ iff $\left\{i \in I \mid x_{i}=y_{i}\right\} \in \mathcal{F}$.

2. With I and $\mathcal{F}$ as before and if $X_{i}=X \forall i \in I$, we denote the ultraproduct $\mathfrak{U}_{i \in I} X_{i}$ by ${ }^{*} X$ and we call it the ultrapower of $X$ after the ultrafilter $\mathcal{F}$.

3. If $\mathrm{T}$ is a set, the extension $\mathcal{U}(T) \stackrel{*}{\hookrightarrow} \mathcal{U}\left({ }^{*} T\right)$, given by $A \mapsto^{*} A$ for any $A \in \mathcal{U}$ is a non-standard enlargement (that is, it satisfies the axioms I,S,T from 7.25 (for $\mathcal{U}(T)$ see 5.16) (note that, for $\mathcal{F}$ a principal ultrafilter we get ${ }^{*} X=X$ for any set $X$, so we do not get anything new in our non-standard 
universe; on the contrary, for $\mathcal{F}$ nonprincipal, it can be shown that ${ }^{*} X=X$ iff $X$ is finite).

Remark 7.41. The sets of numbers extend at ${ }^{*} \mathbf{N} \subset{ }^{*} \mathbf{Z} \subset{ }^{*} \mathbf{Q} \subset{ }^{*} \mathbf{R} \subset{ }^{*} \mathbf{C}$ (certainly, $\mathbf{N} \subset{ }^{*} \mathbf{N}$, $\mathbf{Z} \subset{ }^{*} \mathbf{Z}, \mathbf{Q} \subset{ }^{*} \mathbf{Q}, \mathbf{R} \subset{ }^{*} \mathbf{R}, \mathbf{C} \subset{ }^{*} \mathbf{C}$, the inclusions being strict. However, we will see in 7.45 that, when $I=N$ the fields $\mathbf{C}$ and ${ }^{*} \mathbf{C}$ are isomorphic. We remark again that we produced the set of numbers, and of non-standard numbers starting with the empty set and the axiomatic $Z F(C)$.

Remark 7.42. If all the $X_{i}$ have the same given structure, then, in most of the cases, the same structure lies on their ultraproduct. In particular, when we deal with ultrapowers, one gets, as examples :

1. G group $\Rightarrow{ }^{*} \mathrm{G}$ group.

2. A ring $\Rightarrow{ }^{*} \mathrm{~A}$ ring.

3. $\mathrm{K}$ (really closed, algebraically closed, see $7.16,7.17$ ) field $\Rightarrow{ }^{*} \mathrm{~K}$ (really closed, algebraically cosed) field.

4. V K-vector space $\Rightarrow{ }^{*} \mathrm{~V} * \mathrm{~K}$-vector space.

5. $(M, \leq)$ poset $\Rightarrow\left({ }^{*} M,{ }^{*} \leq\right)$ poset.

6. However, not any property from $\mathcal{U}$ is transferred in ${ }^{*} \mathcal{U}$ as it is. For example $\mathbf{R}$ is Archimedean (i.e. $\mathbf{Z}$ is NOT upperbounded in $\mathbf{R}$ ) but ${ }^{*} \mathbf{R}$ is non-Archimedean ( $\mathbf{Z}$ IS upperbounded in ${ }^{*} \mathbf{R}$ ). However, since the logic from the non-standard enlargement is a higher order logic, something should be transferred. One can say that ${ }^{*} \mathbf{R}$ is ${ }^{*}$-Archimedean (this means that ${ }^{*} \mathbf{Z}$ is NOT upperunbounded in ${ }^{*} \mathbf{R}$ ).

Examples 7.43. If $I=\mathbf{N}$, then an element from $\mathfrak{U}_{n \in \mathbf{N}} X_{n}$ can be written as $\left[x_{1}, x_{2}, \ldots x_{n}, \ldots\right]$ (the class of a sequence). The inclusion $X \subset{ }^{*} X$ is given by the constant sequences $[x, x, \ldots, x \ldots]$, in particular for the inclusion $\mathbf{R} \subset{ }^{*} \mathbf{R}$.

An element $x \in{ }^{*} \mathbf{R}$ such that $x>n, \forall n \in \mathbf{N}$, is called an infinitely big number. An example is $[1,2,3, \ldots, n, \ldots]$ (here $\left[x_{1}, x_{2}, \ldots\right] \leq\left[y_{1}, y_{2}, \ldots\right]$ iff $\left.\left\{i \mid x_{i} \leq y_{i}\right\} \in \mathcal{F}\right)$.

An element $x \in{ }^{*} \mathbf{R}$ such that ${ }^{*}|x|<\epsilon, \forall \epsilon>0, \epsilon \in \mathbf{R}$ is called an infinitely small number (or infinitezimal). An example is $[1,1 / 2,1 / 3, \ldots 1 / n, \ldots]$.

Remark 7.44. In the enlargement $\mathcal{U}(X) \stackrel{*}{\hookrightarrow} \mathcal{U}\left({ }^{*} X\right)$ the standard sets are the ultrapowers, while the internal sets are the ultraproducts which are not ultrapowers, and the external sets are the sets which are not ultraproducts (see 7.30).

Remark 7.45. If $I=\mathbf{N}$, then $\mathbf{C}$ and ${ }^{*} \mathbf{C}$ are both algebraically closed fields (by transfer, $T$ from $I S T$ ) of equal cardinal $>\aleph_{0}$. It results from $7.19,2$. that $\mathbf{C}$ and ${ }^{*} \mathbf{C}$ are isomorphic as fields.

7.46. Non-standard Analysis in SET-type topoi. We start now to achieve our main objective from the mathematical point of view, namely to build a Non-standard Analysis (NSA) in topoi, extending the previous theory from the classical setting (in the topos SET) to more general topoi. We will be only interested in building NSA in the exponential topoi of the form $\mathbf{S E T}^{\mathcal{C}^{o p}}$ (such kind of topoi were also used in Quantum Physics). However, for the moment we will exemplify the ideas on the SET-type topoi 3.- 6. from 6.36. (we will see in the next section that these topoi are of exponential type $\mathrm{SET}^{\mathcal{C}^{o p}}$ ) 
from 6.36. The general definitions will be given in the next section, but a more detailed study of the exponential-type topoi from before will be done in the Part II of this work. For now, let's analyse the already announced examples.

*3. We will define the category ${ }^{*} \mathbf{S E T}{ }^{n}, n \in \mathbf{N}^{*}$. This category is, as is $\mathbf{S E T}^{n}$, a topos. Precisely (the symbol * is exactly the symbol used before for defining the NSA in the topos SET) :

$$
\begin{gathered}
O b\left({ }^{*} \mathbf{S E T}{ }^{n}\right)=\left\{\left({ }^{*} A_{1}, \ldots,{ }^{*} A_{n}\right) \mid A_{i} \in O b(\mathbf{S E T}), i=\overline{1, n}\right\} \\
\operatorname{Hom}^{*} \mathbf{S E T}^{n}\left(\left({ }^{*} A_{1}, \ldots,{ }^{*} A_{n}\right),\left({ }^{*} B_{1}, \ldots,{ }^{*} B_{n}\right)\right)=\left\{\left({ }^{*} f_{1}, \ldots,{ }^{*} f_{n}\right) \mid f_{i} \in \operatorname{Hom}_{\mathbf{S E T}}\left(A_{i}, B_{i}\right), i=\overline{1, n}\right\} \\
{ }^{*} \Omega_{n}=(\{0,1\}, \ldots,\{0,1\})=\Omega_{n},(6.36,3 .) \\
\left({ }^{*} A_{1}, \ldots,{ }^{*} A_{n}\right)\left({ }^{\left(* B_{1}, \ldots,{ }^{*} B_{n}\right)}=\left({ }^{*} A_{1}{ }^{*} B_{1}, \ldots,{ }^{*} A_{n}{ }^{*} B_{n}\right),\right.
\end{gathered}
$$

where ${ }^{*} A_{i}{ }^{*} B_{i}=\left\{f:{ }^{*} A_{i} \rightarrow{ }^{*} B_{i} \mid f\right.$ internal $\}$.

Then $\mathrm{SET}^{n} \stackrel{*}{\hookrightarrow}{ }^{*} \mathrm{SET}^{n}$ and this is a conservative extension of topoi.

More precisely, one can build a NSA on $\mathrm{SET}^{n}$ by imitating the case SET. Namely, in $\mathrm{SET}^{n}$ we define a union, intersection, and power set on components, namely :

$$
\begin{aligned}
& \left(\mathrm{A}_{1}, \ldots, A_{n}\right) \cup\left(B_{1}, \ldots, B_{n}\right):=\left(A_{1} \cup B_{1}, \ldots, A_{n} \cup B_{n}\right) \\
& \left(\mathrm{A}_{1}, \ldots, A_{n}\right) \cap\left(B_{1}, \ldots, B_{n}\right):=\left(A_{1} \cap B_{1}, \ldots, A_{n} \cap B_{n}\right) \\
& \mathcal{P}\left(A_{1}, \ldots, A_{n}\right):=\left(\mathcal{P}\left(A_{1}\right), \ldots, \mathcal{P}\left(A_{n}\right)\right) .
\end{aligned}
$$

Then we can build an Universe $\mathcal{U}(X)$ using the same procedure as in 5.16 (here $X=\left(X_{1}, \ldots, X_{n}\right)$ ). In fact, we get that $\mathcal{U}(X)=\left(\mathcal{U}\left(X_{1}\right), \ldots, \mathcal{U}\left(X_{n}\right)\right)$, where $\mathcal{U}\left(X_{i}\right)$ are Universes as in 5.16. We can also build the non-standard extension $\mathcal{U}(X) \stackrel{*}{\hookrightarrow} \mathcal{U}\left({ }^{*} X\right)=\mathcal{U}\left(\left({ }^{*} X_{1}, \ldots,{ }^{*} X_{n}\right)\right)=\left(\mathcal{U}\left({ }^{*} X_{1}\right), \ldots, \mathcal{U}\left({ }^{*} X_{n}\right)\right)$.

We extend the language to $\mathcal{L}_{\mathcal{U}(X)}$ and we obtain that $\mathcal{L}_{\mathcal{U}(X)}=\left(\mathcal{L}_{\mathcal{U}}\left(X_{1}\right), \ldots, \mathcal{L}_{\mathcal{U}}\left(X_{n}\right)\right)$; this can be further extended to $\mathcal{L}_{\mathcal{U}\left({ }^{*} X\right)}=\left(\mathcal{L}_{\mathcal{U}\left({ }^{*} X_{1}\right)}, \ldots, \mathcal{L}_{\mathcal{U}\left({ }^{*} X_{n}\right)}\right)$. (One can define in $\mathbf{S E T}^{n}$ the notions of standard and non-standard object on components (the standard objects are of the form $\left({ }^{*} X_{1}, \ldots,{ }^{*} X_{n}\right)$, with $X_{1}, \ldots, X_{n}$ sets; the non-standard objects have an hierarchy of non-standardness, depending on how many components are non-standard, and which ones - one can rank them using the lexicographical order on n-uples); one can define, for the non-standard objects, the internal and the external objects, namely an object $\left(X_{1}, \ldots, X_{n}\right)$ is internal iff all the sets $X_{i}$ are internal; and, $\left(X_{1}, \ldots, X_{n}\right)$ is external iff at least one of the $X_{i}$ is external (as in the case of the non-standard sets, one gets several degrees of externality, lexicographically ordered). In fact, the number of degrees of truth of a sentence are $2^{n}$. A sentence in $\mathcal{U}(X)$ consists of an n-uple of sentences from $\mathcal{U}\left(X_{1}\right), \ldots, \mathcal{U}\left(X_{n}\right)$, in this order. The degree of truth of a sentence in $\mathcal{U}(X)$ depends on the number of the true sentences from the components, and on their order, with the lexicographical order on n-uples. The degree of truth of a sentence $\phi$ in $\mathcal{U}(X)$ is kept in $\mathcal{U}\left({ }^{*} X\right)$ and vice-versa, due to $T$ (transfer) and $S$ (standardisation) from the NSA on SET. We got in such a way a conservative extension of the intuitionistic ( $2^{n}$ valued) logic of the topos SET $^{n}$. The idealisation property $(I)$ from NSA remains unchanged. We will better formalise the notions in the next section. We point out that the analogues statements as in 7.21, 7.29, and 7.31 continue to hold, with appropriate definitions of the operations (on components). When considering the maximal extension, at the level of whole topoi $\mathrm{SET}^{n} \stackrel{*}{\hookrightarrow}{ }^{*} \mathrm{SET}{ }^{n}$ it means that we work with the highest possible Universes, 
see 7.24 also.

$\left.{ }^{*} 4\right),{ }^{*} 5$ ). We can apply the same procedure as in *3) for the examples 4) and 5) of 6.36 , building NSA in these topoi also, and keeping the truth values. For the hypercube categories we pass from the functions $f: A_{i} \rightarrow B_{i}$ apearing to the corresponding internal functions * $f:^{*} A_{i} \rightarrow^{*} B_{i}$ and we get the non-standardenlargement $\mathbf{S E T}_{n} \stackrel{*}{\hookrightarrow}{ }^{*} \mathbf{S E T}_{n}$.

${ }^{*} 6$ ). We can apply the same procedure as used in ${ }^{*} 3$. to the example 6 . from 6.36. We get a NSA enlargement of SET-type topoi $\mathcal{E} \stackrel{*}{\hookrightarrow}{ }^{*} \mathcal{E}$ conserving the truth values. By 6.36, 6. for any $n \in{ }^{*} \mathbf{N}$ we can arrange in such a way that the subobjects classifier of $\mathcal{E}$ has exactly $n$ global sections. Then ${ }^{*} \mathcal{E}$ has $n$ truth values also, and the non-standard extension is conservative (keep all the $\mathrm{n}$ truth values unchanged when transferring and when standardizing). We get examples of NSA extensions of an intuitionistic logic having any natural number truth values. We can now shift ourselves from $\mathbf{N}$ to ${ }^{*}$ N. Using the Permanence Principle from classical NSA on SET (see 7.37,1., Overflow Principle) we can build non-standard extensions as before on topoi with hiperfinite number of truth values, see 7.34. However, because there is a bijection (in SET) between the interval $[0,1] \subset \mathbf{R}$, one can think that the set $\{0 / \omega=0,1 / \omega, 2 / \omega, \ldots,(\omega-1) / \omega, \omega / \omega=1\}$ "covers" the continuous set $[0,1]$ (in Quantum Physics the idea in using topoi was to replace the probabilities with truth values).

\section{Infons/Receptons: Theory and Category; Preliminary Definitions}

We already discussed in Section 4. about the fact that any elementary particle from Physics can be thought in String Theory like a coin having two faces, one of them being informational (having the infons as elementary half-particles) and another one being energetic (having the energons as elementary half particles). We also included in Section 4. known comments saying that the energy is also information (see the Landauer Principle [4]), at least at the potential (virtual) level and, in fact, the measurement (observation, reception) produces the collapse of the information waves in the observed reality (such that actual energy and its condensed form, the matter, organized after informational rules in elementary particles - rules which governs the energetic aspects, the atoms, molecules, crystals, human or no-human constructions, plants, animals,...). The information stays in logico-mathematical Theories which "collapse" in Categories (see Section 5.), seen as some kind of concrete - real, material entities, which receive the information; in this case - of Emerging Quantum Mechanics (see [Entropy]) - the two faces become infons/receptons. In Neuroscience one considers that the consciousness is intuitionistic (so, modeled by a multi-valued logic in Heyting algebras), and the union between consciousness and subconsciousness represents a conservative extension of consciousness (it does not change our conscious (aware) feeling on any situation), see the Sections 2. and 3. also. We progressively become aware of our surrounding world, starting from nothingness and progressively receiving and incorporating information (through receptons). Nevertheless, this also happens with the Global Brain, generating progress through brains and technology (and, if we think abouit, the same thing also hold for an Universal Brain, generating the Uni/Multiverse which also contains the life also). The human brain, the global brain (and the universal brain) are higher (and higher) structures, the smaller ones being 
substructures of the bigger ones.

In 5.10 we remembered the Theory of Sets in the standard context of the classical logic $(C L)$ - see 5.9 - and the axiomatic system $Z F(C)$. In 7.25 we extended the notion to its non-standard counterpart, namely using $C L, Z F C$ axiomatics and three more axioms $(S+T, I)$ obtaining the so-called Internal Set Theory (IST), further analysed in $7.26-7.45$. In sections 5. and $7.1-7.15$ we built numbers (and one can continue in the same way with all the mathematics) from nothingness (the empty set, whose existence is axiomatically postulated) and $Z F(C)$ axiomatics in $C L$ (in a given language and using the Modus Ponens deduction rule). So, other categories (and their related theories) arise progressively, as the Universe from the Big Bang. So, a general theory of sets generates many other (non-contradictory, so having models, so generating categories, by 5.19, 5.21) subtheories (we will come back to this notion later) which, in a rank 4 Tegmark' s Multiverse (see section 4.) should have physical models (the additional axiom of a rank 4 Multiverse), which are described by the associated categories. In [GN] the propositional calculus of infons (as defined by them) is a conservative extension of the intuitionistic logic $(C E$ of $I L)$, see 6.1 and 7.25 (in the context of multi-valued logic, conservative means, as in the bivalent case, that the several truth values and their truth order are kept in the extension of the standard sentences, no matter how many they are). Although our furthcoming definition of infon is not the same as given by Gurevich - Neeman, we will keep many similarities, such as the previous description is $(C E$ of $I L$ ). So, what we need now is to reformulate the Set Theory in an intuitionistic setting (obtaining the so-called intuitionistic set theory, based on the named $I Z F$ axiomatic system (see [Stanford]) with $I L)$, and to extend it to its non-standard counterpart - as in the $C L$ case - named Intuitionistic Internal Set Theory IIST. In IZF we get analogues of the theories of IGroups (Intuitionistic Groups), IRings, IVector spaces,..., the difference from the previous ones being that we are not allowed to make deductions using the principle of the excluded middle and analogue arguments (reductio ad absurdum, double negation); precisely, the axioms of Igroups are $I L, I Z F$ (see 8.1) and the axioms of groups (and similarly for the other structures). In IIST we can enlarge, as in the classical case, the previous notions to *IGroups, *IRings, *Vector spaces,... satisfying the axioms of IIST from 8.3 (which naturally extends the axioms of $I S T$ from section 7.), in an $I L$ setting.

Definition 8.1. A set is a class which is an element of another class (see 5.10). Let's denote by $\mathcal{M}$ the class of sets. The intuitionistic theory of sets $(I Z F)$ is the triplet $\left(\mathcal{L}_{\mathcal{M}}, I L+I Z F, \mathcal{D}=\{M P\}\right)$, where $I L$ are the axioms from 6.1, $M P$ is as in 5.7, and $I Z F$ (Intuitionistic Zermelo-Fraenkel) consists in the following axioms (recall that " $\in$ " and "=" are primary relations)

$I Z F$ : Axioms (from 5.10): 1.(E), 3.(S), 4.(P), 5.(U), 6.(Rp), 7.(I), 8.(PS), and (2)'(Induction):

$$
\forall x((\forall y \in x \wedge \phi(y)) \rightarrow \phi(x)) \rightarrow \forall x \phi(x)
$$

for every formula $\phi$.

Remarks 8.2. The axioms 2.(R) and 9.(C) from 5.10 implies the law of excluded middle (see [https://plato.stanford.edu/entries/set-theory-constructive/incompatible-set-axs.html]), which is forbidden in $I L$ which is not $C L$ (it is not hard to see this, but we do not insist here). This is the reason 
for which the axiom 2. of $Z F$ is weakened to 2'. from before, and 9. is not included (however, weaker versions of $\mathbf{C}$ could be encluded, as is the Axiom of countable choice, for instance).

Definition 8.3. An Intuitionistic Theory is an usual theory $\mathcal{T}=(\mathcal{L}, \mathcal{A}, \mathcal{D})$ (see 5.8) in an intuitionistic context, so assuming that $\mathcal{A}$ contains the axioms $I L$ and $I Z F$ from before (strictly, with equality only in the case 8.1 from before), and that $\mathcal{D}=\{M P\}$ (see 5.7). As in 5.8, we assume, by definition, that a theory has at least one model.

Remark 8.4. Let $\mathcal{E}$ be a topos. In section 6. we defined all the logical operations in the space of sections $\operatorname{Hom}_{\mathcal{E}}(1, \Omega)$, see 6.43 and 6.52, such that this space become a $M H A$ (see 6.50). Hence, we can consider the notion of theory (in the sense of the previous definition) in any topos.

Definition 8.5. Let $\mathcal{C}$ be a category, and $A \in O b \mathcal{C}$. We say that $B \in O b \mathcal{C}$ is a subobject of $\mathrm{A}$ (in $\mathcal{C}$ ) if there is a monomorphism $B \hookrightarrow A \in \operatorname{Hom}_{\mathcal{C}}(B, A)$. We say that a category $\mathcal{C}^{\prime}$ is a subcategory of $\mathcal{C}$, if $\mathcal{C}^{\prime}$ is a subobject of $\mathcal{C}$ in a category CAT as in 5.25, containing as objects both $\mathcal{C}$ and $\mathcal{C}^{\prime}$.

Definition 8.6. Let $\mathcal{E}$ be a topos, and $X \in O b(\mathcal{E})$. Then we can associate to any two morphisms $\alpha \in \operatorname{Hom}_{\mathcal{E}}(1, X)$ and $\beta \in \operatorname{Hom}_{\mathcal{E}}(1, X)$ their direct product $(\alpha, \beta) \operatorname{inHom}_{\mathcal{E}}(1, X \times X)$ (1 is (the) final object, so $1 \times 1=1$ ), see 6.14 . Then, a binary relation $\mathcal{R}$ on $\mathrm{X}$ is a set of pairs $(\alpha, \beta)$ as before. As usually, we write $\alpha \mathcal{R} \beta$ instead of $(\alpha, \beta) \in \mathcal{R}$. We say that a binary relation on $\mathrm{X}$ in finitely satisfiable iff for any positive integer $n$ and $\alpha_{1}, \ldots, \alpha_{n} \in \operatorname{Hom}_{\mathcal{E}}(1, X)$ there is $\beta \in \operatorname{Hom}_{\mathcal{E}}(1, X)$ such that $\alpha_{1} R \beta, \ldots, \alpha_{n} R \beta$.

Definition 8.7. Let $\mathcal{E}$ be a topos. An enlargement of $\mathcal{E}$ is a monomorphism ${ }^{*}: \mathcal{E} \hookrightarrow^{*} \mathcal{E}$ in a category CAT containing both categories $\mathcal{E}$ and ${ }^{*} \mathcal{E}$, satisfying :

- 1. * $\mathcal{E}$ is a topos;

- 2. If $\mathbf{V}: \mathcal{P} \rightarrow \operatorname{Hom}_{\mathcal{E}}(1, \Omega)$ is any valuation (see 6.46) (here $\mathcal{P}$ is the set of sentences in the language $\mathcal{L}_{\mathcal{E}}, 1$ is the final object is $\mathcal{E}$, and $\Omega$ is the subobjects classifier from $\left.\mathcal{E}\right)$, then ${ }^{*} \mathbf{V}\left({ }^{*} \mathrm{p}\right)=\mathbf{V}(\mathrm{p})$ for any $p \in \mathcal{P}$ (here ${ }^{*} \mathbf{V}:{ }^{*} \mathcal{P} \rightarrow{ }^{*} \operatorname{Hom}_{* \mathcal{E}}\left(1,{ }^{*} \Omega\right)$ is the non-standard image of the original map $\mathbf{V}, 1={ }^{*} 1$ is the final object, and ${ }^{*} \Omega$ is the subobjects classifier from ${ }^{*} \mathcal{E}$ );

- 3. For any $X \in O b(\mathcal{E})$ and any finitely satisfiable relation $R$ on $\mathrm{X}$ there is $g \in{ }^{*} H o m_{* \mathcal{E}\left(1,{ }^{*} X\right)}$ such that $\alpha^{*} R g$ for any $\alpha \in H o m_{\mathcal{E}}(1, X)$ (we say that any finitely satisfiable relation is globally satisfiable) (if $R \subseteq \operatorname{Hom}_{\mathcal{E}}(1, X \times X)$ then ${ }^{*} R \subseteq{ }^{*} \operatorname{Hom}_{*} \mathcal{E}\left(1,{ }^{*} X \times{ }^{*} X\right)$ ).

Remark 8.8. The previous definition extends to topoi the axioms I, S, T from 7.25. The extension ${ }^{*}: \mathcal{E} \hookrightarrow{ }^{*} \mathcal{E}$ preserves the truth values (by $8.7,2$ ) so it is a conservative extension in an intuitionistic setting. If we produce an arrow ${ }^{*}$ as in 8.7 , we get models for the already announced IIST. However, it is not known if such an arrow exists for a general topos (for some results see [nlab]). We will be interested only in the topoi of the form $\mathbf{S E T}^{\mathcal{C}^{o p}}$ (of exponential type). However we will consider the exponential case in part II of this work, as already announced, in this section we will work only with 
SET-type topoi (see 5.35).

As anounced (and justified) in section 4., we are now prepared to define the infons and the receptons. Precisely :

Definition 8.9. We call a generalised infon a Theory $\mathcal{I}=(\mathcal{L}, \mathcal{A}, \mathcal{D})$ whose propositional calculus is a conservative extension of the intuitionistic logic. We call infon the collection of entities of the previous form, modeled by the theory of non-standard intuitionistic axiomatics (IIST) whose category of models form a non-standard enlargements of a topos of the form $\mathrm{SET}^{\mathcal{C}^{o p}}$, where $\mathcal{C}$ is any category (see Section 5. also). We call preliminary defined infon the infon whose associated category is a non-standard enlargement of a topos of SET-type (this means topoi of the form ${ }^{*} 3 .-^{*} 6$. from 7.46).

Definition 8.10. We call a generalized recepton the category of models of a generalized infon. We call recepton any non-standard enlargement of a topos of exponential type - $\mathrm{SET}^{\mathcal{C}^{o p}}$, where $\mathcal{C}$ is any category (see Section 5. also). We call preliminary defined recepton any non-standard enlargement of a topos of SET-type (as before, these are the topoi of the form ${ }^{*} 3 .{ }^{*} 6$. from 7.46 ; we note that, by the just given definition of the recepton we need to show that the previous topoi 3.- 6. from 6.36 are of the form $\mathbf{E N S}^{\mathcal{C}^{o p}}$ ).

We recall in the context that the logic from topoi is intuitionistic, and its non-standard enlargement is a conservative extension (see Sections 6. and 7.).

Examples 8.11. (of preliminary defined receptons). We built in Section 7. concrete enlargements of the categories $\mathbf{S E T}^{\mathbf{n}}, \mathbf{S E T}^{\mathbf{n}_{1}, \ldots, \mathbf{n}_{\mathbf{s}}}$ and $\mathbf{S E T}^{\mathbf{n}}$ with several inclusions (6.36, 3.-6.), namely ${ }^{*} 3 .-^{*} 6$. (see 7.46).The objects of these two categories are examples of receptons as long as we prove that the categories from 6.36 are of the type $\mathrm{SET}^{\mathcal{C}^{o p}}$ for some category $\mathcal{C}$ (see 8.10). We will analyse those categories successively.

3. Let's organize the set $\mathbf{n}=\{1,2,3, \ldots, n\}$ as a category, whose objects are the numbers $k$, and the only arrows are the identities of each $k, 1 \leq k \leq n$ (a discrete category). The category $\mathbf{n}$ coincides with dual (it is selfdual). Then $\mathbf{S E T}^{n}=\mathbf{S E T}^{\mathbf{n}}$ (the first $\mathbf{n}$ is the number, and the second $\mathbf{n}$ is the category), because any object of $\mathbf{S E T}^{\mathbf{n}}$, meaning a functor $F: \mathbf{n} \rightarrow \mathbf{S E T}$ can be identified with $(F(1), F(2), \ldots, F(n)) \in O b\left(\mathbf{S E T}^{n}\right)$ and any morphism in $\mathbf{S E T} \mathbf{T}^{\mathbf{n}}$, meaning a natural transformation between two functors $F, G \in \mathbf{S E T}^{\mathbf{n}}$ can be identified with n arrows $f_{i}: F(i) \rightarrow G(i), i=\overline{1, n}$ from SET i.e. an arrow in $\mathrm{SET}^{n}$.

4. As for $\mathbf{S E T}^{n_{1}, \ldots, n_{s}}$, for $n_{1}<n_{2}<\ldots<n_{s}, s \in \mathbb{N}^{*}, n_{i} \in \mathbb{N}, i=\overline{1, n}$, let's consider the direct sum category $\mathbf{n}_{\mathbf{1}}+\mathbf{n}_{\mathbf{2}}+\ldots+\mathbf{n}_{\mathbf{s}}$ whose objects are $(k, j), 1 \leq k \leq n_{j}, j=\overline{1, s}$ and the morphisms are only the identities (so the direct sum category is discrete again). Then $O b\left(\mathbf{S E T}^{n_{1}, \ldots, n_{s}}\right)=\left\{\left\{\left(A_{11}, A_{21}, \ldots, A_{n_{1} 1}\right),\left(A_{12}, A_{22}, \ldots, A_{n_{2} 2}\right), \ldots,\left(A_{1 s}, A_{2 s}, \ldots, A_{n_{s} s}\right)\right\} \mid A_{i j} \in\right.$ $\left.\mathrm{Ob}(\mathbf{S E T}), j=\overline{1, s}, i=\overline{1, n_{j}}\right\}$. Let's denote by $f_{j}: \mathbf{n}_{\mathbf{j}} \rightarrow \mathbf{n}_{\mathbf{1}}+\mathbf{n}_{\mathbf{2}}+\ldots+\mathbf{n}_{\mathbf{s}}, j=\overline{1, s}$ the canonical monomorphisms (injections) coming from the universal propriety of the direct sum. We show that the category $\mathbf{S E T}^{n_{1}, \ldots, n_{s}}$ can be identified with the category $\mathbf{S E T}^{\mathbf{n}_{1}+\ldots+\mathbf{n}_{\mathbf{s}}}$, which is of the desired type. Indeed, as in 3., each $n_{j}$-uplu $\left(A_{1 j}, A_{2 j}, \ldots, A_{n_{j} j}\right)$ uniquely define a functor $F_{j} \in \mathbf{S E T}^{\mathbf{n}_{\mathbf{j}}}$. Then 
consider $F \in \mathbf{S E T}^{\mathbf{n}_{1}+\ldots+\mathbf{n}_{\mathbf{s}}}, F=F_{1}+\ldots+F_{s}$ the direct sum functor (induced by the prpriety of universality of the direct sum), so $F \circ f_{j}=F_{j}, j=\overline{1, s}$. In fact, $F(k, j)=F_{j}(k), k=\overline{1, n_{j}}\left(=A_{k j}\right)$. As for the fact that the natural transformations between the functors from $\mathrm{SET}^{\mathbf{n}_{1}+\mathbf{n}_{2}+\ldots+\mathbf{n}_{\mathbf{s}}}$ corresponds exactly to the morphisms from $\mathbf{S E T}^{n_{1}, \ldots, n_{s}}$, use the same observation as we did at 3. See also the next diagram:

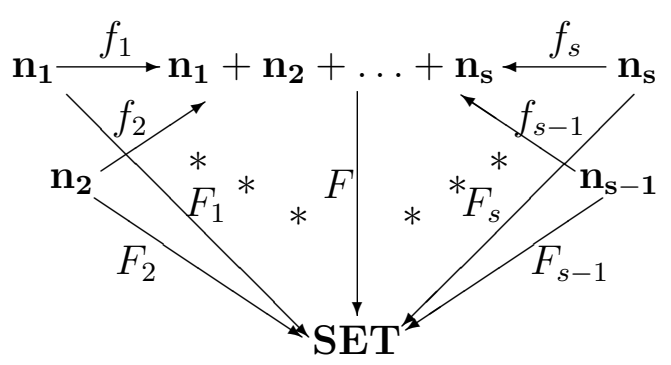

5.,6. The categories of this type from 6.36 are similar to the category $\mathbf{S E T}^{n}$ from the previous example 3., but having some inclusions among the $A_{i}$ 's. In fact, from the point of view of the truth values, everything will work even if instead of an inclusion $A_{i} \subset A_{j}$ we request to have a morphism $f_{i j}: A_{i} \rightarrow A_{j}$. However, we will not be able to identify the category obtained replacing the inclusion with a morphism with a category of the type $\mathrm{SET}^{\mathcal{C}^{o p}}$, except for the case when we request that the morphism is a monomorphism, but this gives us the inclusion case, by 6.34. We proceed as in 3., but we will no more consider the discrete structure in the category whose objects are $\{1,2, \ldots, n\}$. Instead, we consider additional morphisms between these objects. Precisely, the morphisms in the new category having the same objects as the discrete category $\mathbf{n}$ will be, certainly, all the identities, but we add an epimorphism $j \rightarrow i$ whenever in our examples we have $A_{i} \subset A_{j}$ (note that the non-discrete category is no more self-dual, so we have to reverse the rows and to change monomorphisms in epimorphisms). For example, if one considers the category $\mathrm{SET}_{2}^{4}$, take the category $\mathbf{4}_{0}$ whose objects are the same as for the category $\mathbf{4}$, namely $\{1,2,3,4\}$, but whose morphisms are: $\left\{\mathbf{1}_{1}, \mathbf{1}_{2}, \mathbf{1}_{3}, \mathbf{1}_{4}, 2 \rightarrow 1,4 \rightarrow 3,3 \rightarrow 1,4 \rightarrow 2\right.$, the last four being epimorphisms $\}$ (note that the category $\mathbf{4}_{0}$ is not self-dual). Then one can prove, using the methods from before, that $\mathrm{SET}_{2}^{4}=\mathrm{SET}^{4_{0}}$, so of the desired type.

8.12. As we already mentioned, the Nicolas Bourbaki group of prominent mathematicians from France considered that the fundamental structures in mathematics are (both in the language of theories and of categories) the algebraic structures, topological structures, and order structures, all the others (like the differential ones, for example) being combinations between them. So, in order to apply Mathematics in various arias, one needs to consider collections of such structures, communicating between them. This means that we need to consider various theories and categories together. In the case of categories, the structures are collected in categories, and the communication between them is done with the help of the functors. This means that, in fact, one needs to work with categories of type CAT (see 5.25), and, as a quonsequence, with appropriate infons and reeptons. This means that an important family of receptons will have the form SET $\mathbf{C A T}^{\text {op }}$ and similarly for the corresponding theories. For example, in Multiversal Quantum Physics several mathematical structures appearing in the usual Quantum Physics were put together in such kind of topoi several, such as Hilbert spaces with compact self adjoint operators, von Neumann algebras, and others. However, when studying the human and global brain, is necessary to 
enlarge the things to the non-standard environment, due to the role played by the subconsciousness.

8.13. There are infons which have more information than others, as well as they can have each one different kinds of information. We say that a theory $\mathcal{T}_{1}$ is a subtheory of the theory $\mathcal{T}_{2}$ iff the constants of $\mathcal{L}_{1}$, belonging to $\mathcal{T}_{1}$ has the variables and the constants among those of $\mathcal{L}_{2}$, belonging to $\mathcal{T}_{2}$, the axioms of $\mathcal{T}_{1}$ contains the axioms of $\mathcal{T}_{2}$, and the set of deduction rules of $\mathcal{T}_{1}$ contains the set of deduction rules of $\mathcal{T}_{2}$. The biggest theory is the Theory of sets, (Set). All the other theories are subtheories of this one. We can, then, organize the theories (as subtheories of $(\operatorname{Set}))$ in a POSET $(\operatorname{Sub}(\operatorname{Set}), \leq$ ). In particular this structure is transmitted to the infons. We point out that the non-standard intutionistic theories, as we built them here in examples, are also subtheories of (Set). As for receptons, being the categories associated (through models) to the infons, they also gave to the highest category, namely SET a structure of POSET, via subcategories. So we get the partial ordered set $(\mathrm{SET}, \leq)$. These structures will be studied in more detail in part II., when we will also have the construction of the infons and receptons in general.

8.14. Oriented graphs as topoi of SET-type. An oriented graph $\overrightarrow{\mathcal{G}}$ consists in a finite number of points $A=\left\{A_{1}, A_{2}, \ldots, A_{m}\right\}$ (in the plane, space, or even in a higher-dimensional space - although we can always to project them on the plane, obtaining a planar graph), some (or all) of them being joined with some others (or all) of them by oriented segments (arrows). We will suppose here, additionally, that for each $i \neq j, i, j \in\{1, \ldots, n\}$ there is at most on arrow between them, either from $A_{i}$ to $A_{j}$ or from $A_{j}$ to $A_{i}$, and we implicitely assume the existence of an unique arrow from $A_{i}$ to itself, for any $i$ as before, namely the identity arrow (which has no orientation). As we saw before, we can organize this graph as a category of type $\mathbf{n}$ with some aditional morphisms (see $8.11,3$. - 6.): we add to $\mathbf{n}$ a morphism from $j$ to $i, i, j \in \mathbf{n}$ iff there is an arrow from $A_{i}$ to $A_{j}$ in $\overrightarrow{\mathcal{G}}$, as in 5. and 6. from 8.11. let's denote this new category by $\mathbf{n}_{\overrightarrow{\mathcal{G}}}$. Then the $\mathbf{S E T}$-type topos $\mathbf{S E T}_{\overrightarrow{\mathcal{G}}}$ can replace the given graph. In fact one can work with a category of n-uples of sets, $\left(X_{1}, \ldots, X_{n}\right)$ requesting additionally that $X_{i} \subset X_{j}$ whenever there is an arrow from $A_{i}$ to $A_{j}$ in $\overrightarrow{\mathcal{G}}$. This approach can be used to study various kind of graphs, in particular graph neural (quantum) networks of various kinds (optical, convolutional,...) with the quantum aspects modeled in topoi. Such a development is related to deep learning, artificial intelligence, quantum computers (starting with the quantum Turing mashine). Moreover, Mathematical Game Theory is also related to Graph Theory, and some economical applications can be obtained, for instance related to the new aria of Neuroeconomics. There are also connections between graphs and the evolutionary games (with applications in the study of climate change, evolutionary biology,...).

8.15. Geometric topoi. One can use the previous tops-interpretation of the oriented graphs to build Geometric Topoi. One example is the topos from 5.33 (see 6.36, 5.also), the hypercube category $\mathbf{S E T}_{n}$. We can also define a hypertetrahedron category $\mathbf{T E T}_{n}$. The general rule consists in considering oriented tetrahedra $A_{1} A_{2} A_{3} A_{4}$ (if one speaks about the 3D tetrahedron), as in the next figure : 


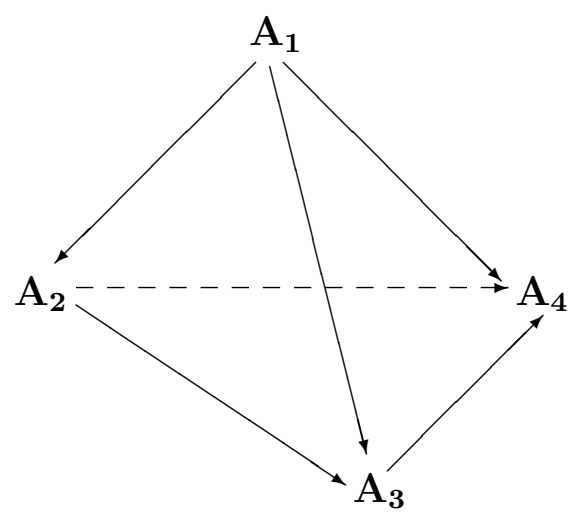

We denote now by $\overrightarrow{\mathcal{T}}$ the oriented graph given by the previous tetrahedron, and by $\mathbf{n}_{\overrightarrow{\mathcal{T}}}$ the discrete category $\mathbf{n}$ at which we added the additional morphisms $2 \rightarrow 1,3 \rightarrow 1,4 \rightarrow 1,3 \rightarrow 2,4 \rightarrow 2,4 \rightarrow 3$. Then the previous tetrahedron can be recognized in the topos $\mathbf{S E T}_{\overrightarrow{\mathcal{T}}}$ or, alternatively, in the topos whose objects are the quadruplets $\left\{\left(A_{1}, A_{2}, A_{3}, A_{4}\right) \mid A_{1}, A_{2}, A_{3}, A_{4}\right.$ sets, with $\left.A_{1} \subset A_{2}, A_{1} \subset A_{3}, A_{1} \subset A_{4}, A_{2} \subset A_{3}, A_{2} \subset A_{4}, A_{3} \subset A_{4}\right\}$ and having as morphisms quadruplets of functions, as in 6.36, 5. In such a way, one can represents in topoi any polyhedron from $\mathbf{R}^{3}$ and, in fact, any polytope from $\mathbf{R}^{n}, n \geq 3$.

8.16. Gosset polytope. An important polytope in Emergent Quantum Mechanics (where the consciousness become part of Physics) is the Gosset polytope (see the final part of section 4.). It is a polytope $P \subset \mathbf{R}^{8}$ having 240 vertices, with coordinates $( \pm 2, \pm 2,0,0,0,0,0,0)$, with any combination of signs and the 2's on any two positions (112 vertices) and ( $\pm 1, \pm 1, \pm 1, \pm 1, \pm 1, \pm 1, \pm 1, \pm 1$ ), by taking an even number of minus signs (128 more vertices). The vertices of the analogue of the Gosset polytope in $\mathbf{R}^{3}$ has the coordinates $( \pm 2, \pm 2,0),( \pm 2,0, \pm 2),(0, \pm 2, \pm 2)$ (12 vertices), and $(-1,1,1),(1,-1,1),(1,1,-1),(-1,-1,-1)$ so, this polyhedron has 16 vertices. They form 3 squares of radious 4 , with the centers in the center of the axes of coordinates and sitting in the planes $\mathrm{xOy}, \mathrm{yOz}$, $\mathrm{zOx}$ respectively ( $\mathrm{O}$ is the center of the axes), and having the edges parallel with the axes of coordinates, and a regular tetrahedron. The 12 vertices of the three squares are the middle of the edges of a cube having edges of length 4 , the center in $\mathrm{O}$, and the faces paralel with the planes of coordinates. So, this polyhedron is determined by a cube and a (regular) tetrahedrun. It can be represented as a topos using the remarks from 8.15 and 6.36, 5., first exmple. But even the Gosset polytope from $\mathbf{R}^{8}$ can be represented in such a way once we give orientations to its edges. However, the group of symmetries of the Gosset polytope is a group which can be found in the structure of the (-2) curves from a degenerate del Pezzo surface of degree d. However, we will deal with this subject in furthcoming paper, based on [P1], [P2].

\section{The Yang-Baxter equations}

In Part II of this paper (Artificial Intelligence) we will present in more detail the connections between DNA, Quantum Computers and the sequence knots, braid goups and Yang-Baxter equations (YBE). Here we begin with an introduction to the Yang-Baxter equation. After that we will present related results and applications of this equation.

Let $V$ be a vector space over $k$. Let $I=I_{V}: V \rightarrow V$ be the identity map of the space $V$. 
We denote by $\tau: V \otimes V \rightarrow V \otimes V$ the twist map defined by $\tau(v \otimes w)=w \otimes v$.

Now, we will give the main notations for introducing the Yang-Baxter equation.

For $R: V \otimes V \rightarrow V \otimes V$ a $k$-linear map, let $R^{12}=R \otimes I, R^{23}=I \otimes R: V \otimes V \otimes V \rightarrow V \otimes V \otimes V$. In a similar manner, we denote by $R^{13}$ a linear map acting on the first and third component of $V \otimes V \otimes V$. It turns out that $R^{13}=(I \otimes \tau)(R \otimes I)(I \otimes \tau)$.

Definition 9.1 $A$ Yang-Baxter operator is k-linear map $R: V \otimes V \rightarrow V \otimes V$, which satisfies the braid condition (the Yang-Baxter equation):

$$
R^{12} \circ R^{23} \circ R^{12}=R^{23} \circ R^{12} \circ R^{23} .
$$

We also require that the map $R$ is invertible.

Remark 9.2 Some examples of Yang-Baxter operators are the following: $R=\tau$ (i.e., $R(a \otimes b)=b \otimes a$ ), and $R=I \otimes I$ (i.e., $R(a \otimes b)=a \otimes b$ ).

Remark 9.3 An important observation is that if $R$ satisfies (1) then both $R \circ \tau$ and $\tau \circ R$ satisfy the QYBE (the quantum Yang-Baxter equation):

$$
R^{12} \circ R^{13} \circ R^{23}=R^{23} \circ R^{13} \circ R^{12} .
$$

Remark 9.4 The equations (1) and (2) are equivalent.

The Yang-Baxter equation is an important tool for the theory of quantum groups, knot theory, the theory of universal quantum gates and the theory of quantum computers (see [1,14,15,17-19]).

Remark 9.5 There is a similar terminology for the set-theoretical Yang-Baxter equation. In this case $V$ is replaced by a set $X$ and the tensor product by the Cartesian product. We will explain this definition in the next examples below.

Remark 9.6 Let $X$ be a set containing three logical sentences $p, q, r$ (i.e., $p, q, r \in X$ ). We can choose $X$ as rich as we wish for the moment. Later, we will try to find the smallest $X$ which fits for our theory.

Let $R: X \times X \rightarrow X \times X$, be defined by $R(p, q)=(p \vee q, p \wedge q)$.

It follows that

$$
(R \times I) \circ(I \times R) \circ(R \times I)=(I \times R) \circ(R \times I) \circ(I \times R) .
$$

One way to check that (3) holds is to make a table with values for $p, q, r$.

Remark 9.7 Another interesting solution to the set-theoretical Yang-Baxter equation is the following.

Let $R: X \times X \rightarrow X \times X$, be defined by $R(p, q)=(p \rightarrow q, p)$.

Again, one way to check the above statement is to make a table with values for $p, q, r$. 
Let us denote $R(p, q)=\left(p_{1}, q_{2}\right)$. The set-theoretical Yang-Baxter equation can be expressed as $\left(p_{101}, q_{212}, r_{020}\right)=\left(p_{010}, q_{121}, r_{212}\right)$.

It is now the moment to discuss about $X$.

What can be said about $X$ in general (what is the smallest $X$ for which $R$ is well-defined)? One could consider a set $X$ containing more than three logical sentences. What is the interpretation of the set-theoretical Yang-Baxter solutions in this case ?

For example, if $\mathcal{B}$ is a Boolean topos, the pairs from 9.7 gives solutions for the YBE (1) from the global sections of subobjects classifier $\Omega$ (i.e. $\operatorname{Hom}_{\mathcal{B}}(1, \Omega)$ ) of $\mathcal{B}$, while the pairs from 9.6 gives solutions from the global sections of subobjects classifier $\Omega$ (i.e. $\operatorname{Hom}_{\mathcal{E}}(1, \Omega)$ ) for both equations YBE and QYBE ((1) and (2)) and for any topos $\mathcal{E}$.

We can go a step further and consider an algebra of type $(2,2),(A, *, \circ)$, and call the operations $*$ and $\circ Y B$ conjugated if $R(a, b)=(a * b, a \circ b)$ satisfies the set-theoretical Yang-Baxter equation.

We propose the study of algebras with two operations of type $(2,2)$ which are YB conjugated. (Distributive lattices, groups and self-distributive structures fall into this category.)

Remark 9.8 We now consider other equations for a $k$-linear map $R: V \otimes V \rightarrow V \otimes V$ :

$$
\begin{gathered}
R^{12} \circ R^{23}=R^{13} \circ R^{12}=R^{23} \circ R^{13} \\
R^{23} \circ R^{12}=R^{12} \circ R^{13} \\
R^{12} \circ R^{13} \circ R^{12} \circ R^{23}=R^{13} \circ R^{23} \circ R^{13} \circ R^{12} \\
\left(R^{12} \circ R^{23} \circ R^{12}-R^{23} \circ R^{12} \circ R^{23}\right) \circ\left(R^{12} \circ R^{13} \circ R^{23}-R^{23} \circ R^{13} \circ R^{12}\right)=0
\end{gathered}
$$

Theorem 9.9 If a k-linear map $R: V \otimes V \rightarrow V \otimes V$ verifies (4) and (5) then $R$ is a common solution for (1) and (2).

If a $k$-linear map $R: V \otimes V \rightarrow V \otimes V$ verifies (1) and (2) then $R$ is a solution for (6).

If a $k$-linear map $R: V \otimes V \rightarrow V \otimes V$ verifies (1) or (2) then $R$ is a solution for (7).

Proof. We only prove the first claim: $R^{23} \circ R^{12} \circ R^{23}=R^{23} \circ R^{13} \circ R^{12}=R^{12} \circ R^{23} \circ R^{12}$ $R^{23} \circ R^{13} \circ R^{12}=R^{23} \circ R^{12} \circ R^{23}=R^{12} \circ R^{13} \circ R^{23}$

The other claims follow in a similar manner. $\diamond$

We will write the above results in the following manner:

$(4) \wedge(5) \rightarrow(1) \wedge(2)$;

$(1) \wedge(2) \rightarrow(6)$

$(1) \vee(2) \rightarrow(7)$.

As a direct application of this theorem, one can check which of the funtions presented in this section are common solutions for the braid condition and the quantum Yang-Baxter equation.

We are now preparing the framework for the next theorem. 
For an equation $p$ (for example, $R^{12} \circ R^{23} \ldots=0$ ), we can associate $\neg p$ (for example, $R^{12} \circ R^{23} \ldots \neq 0$ ).

Now we can consider the equation $\top$ the equation always true $(p \vee \neg p)$ and by $\perp=\neg(\top)$ the equation with no solutions.

We now can state a theorem with applications in the understanding the theory of the Yang-Baxter equation.

Theorem 9.10 The set of equations of operators of type $R: V \otimes V \rightarrow V \otimes V$ and $R^{i j}: V \otimes V \otimes V \rightarrow$ $V \otimes V \otimes V$, with $i, j \in\{1,2,3\}$, has a natural distributive latice structure. In this lattice, one can define a partial order, a minimum $\perp$, a maximum $\top$, and operations $\neg$ and $\rightarrow$.

\section{Conclusions and Further Considerations}

Concluding, in this paper we get three kinds of contributions. One is related to pure mathematics by constructing non-standard analysis in the SET-type topoi, that is the topoi of the form $\mathbf{S E T}_{\overrightarrow{\mathcal{G}}}$ (this follows from 5.34, 5. and 8.11, 3 and 7.46, ${ }^{*} 3$ and 8.14). Another one is the interpretation of these mathematical theories in terms of infons and receptons, see section 8. And another one consists in contributions to the theory of Yang-Baxter equations and their connections with topoi. Moreover, the exemple 5.20, 6. is proved for the first time (although it was mentioned, without proof in [16]).

As we pointed out several times, we continue our work in part II. Artificial Intelligence. We will give the general construction for receptons, and we will start to study the Global Brain, consisting of all the human brains and the technologies (artificial intelligence for instance). We will try to develop a topoi-theoretic approach to the study of the neural networks and to the study of the Turing machines. As for the brain, both individual and global, there exists some partial mathematical/physical models, the Ising model (based on statistical Physics), and the Quantum model, based on Quantum Physics (Penrose - Hameroff). In both models the methods of topoi theory could play an important role, both models being related to the notion of probability, which could be replaced by truth values in topoi (in an intuitionistic context).

On the other hand, it is necessary to deal with infinite graphs also. For example R. Aumann introduced the notion of continuous economies ([Au]), meaning that the number of players is in bijection with the set of real numbers. So, thinking as in 8.14 , we arrive to graphs with a continuum of nodes. In order to model such graphs, which allows us to use other kind of mathematical methods which are defined over the set of real numbers - such as differential, integral and variational calculus, differential and partial order differential equations, etc. - avoiding in such a way problems from discrete mathematics related to the complexity of the computations, we need to study general topoi of the form $\mathbf{E N S}^{\mathcal{C}^{\circ p}}$, seen as receptons, for arbitrary categories, not for finite categories only, as we did in section 8 . Another reason for which Aumann introduced the continuous economies was generated by the fact that the brain of the employee from Whashington (this is Aumann's example) perceive the very many small economic entities as a continuum; they produce $1 / 3$ of the economy of USA, so they cannot be neglected. On the other hand, Aumann itself gave a counter-example to this approach in the same paper ([3]), producing an example of a theorem true for continuous economies, but false for any finite economy, doesn't matter how big or how small they are (in the finite case an additional hypothesis is necessary). So, the theory of continous economics (and, by similarity, of continous mechanics) does not always give 
theorms applicable in decisions. We need to test somehow the applicability, and this can be done using classic non-standard analysis. Precisely, let's consider a theorem, true over an $\mathbf{R}$ of players. If we transfer it to ${ }^{*} \mathbf{R}$ it remains true ( $\mathrm{T}=$ transfer, see 7.25 ). If its restriction to ${ }^{*} \mathbf{N}$ (hypernatural numbers) remains true for all hiperfinite number of players, smaller than some fixed hyperfinite natural number, then the theorem holds for sufficiently large (finite) economies, by 7.37, 2) (Underflow), otherwise don't (Overflow). Hence, in the first case only, the theorem can be used for economical decisions. We call this the hyperfinite test. If we interpret as before the continuous economies in topoi of the form $\mathbf{S E T}^{\mathcal{C}^{o p}}$, we need a kind of hyperfinite test in these topoi, so we need non-standard enlargements in SET ${ }^{\mathcal{C}^{o p}}$.

On the other hand, although it seems that we do not need a non-standard enlargement for Artificial Intelligence, it is part of the Global Brain, which has, as the human brain has, consciousness and subconsciousness, so, as in the case of the human brain, we need again non-standard extensions in exponential-type topoi. We will consider such kind of topics in Part II.

Aknowledgement. The authors of this paper had fruitful scientific discussions with Prof. Leon Zăgrean (Faculty of General Medicine, Bucharest) for neuroscience and Drd. Ileana Ruxandra Badea (Faculty of Economic Cybernetics, Statistics, and Informatics) for artificial intelligence, although the two domains cannot be separated in this work; they played an important role in both parts of this work.

\section{References}

1. Alagic, G.; Bapat, A.; Jordan, S. Classical simulation of Yang-Baxter gates. Mathematics 2014 http://arxiv.org/abs/1407.1361.

2. Anderson, R.M. Non-standard analysis with applications to economics, in Handbook on Mathematical Economics, vol IV, Elsevier, 1991.

3. Aumann, R., Markets with a Continuum of Traders, Econometrica, 34(1964) nr 1-2, pp. 39-50.

4. Bomarshenko, E. The Landauer Principle: Re-Formulation of the Second Thermodynamics Law or a Step to Great Unification ?, Entropy 2019, 21, 918, MDPI, pp. 2-6.

5. Chang, C.C., Keisler, H.J. Model Theory, 3rd ed., Studies in Logic 73(1992), North-Holland.

6. Doring, A.,Isham, C., Classical and Quantum Probabilities as Truth Values, arXiv:1102.2213v1 quant-ph, 2011.

7. Eagleman, D. The Brain: The Story of You, Canongate Books Ltd., 2015.

8. Everett, H., The Theory of the Universal Wavefunction, in Many-worlds Interpretation for quantum mechanics, Princeton Univ. Press, 1979, pp. 3-140.

9. Flori, C. Lectures on Topos on Quantum Theory, Graduate Course 2012, Arxiv 1207.1744, 2012.

10. Goldblatt, R. Topoi: the Categorial Analysis of Logic, North-Holland, 1984.

11. Goldblatt, R. Lectures on the Hyperreals, Springer, 1998.

12. Greene, B., The Elegant Universe, W.W.Norton, 1999.

13. Gurevich, Y. , Neeman, I. The logic of infons: the Propositional Case, ACM Transactions on Computational Logic, Vol. 12, No. 2 (2011). 
14. Iordănescu, R.; Nichita, F.F.; Nichita, I.M. The Yang-Baxter Equation, (Quantum) Computers and Unifying Theories, Axioms 2014, 3,pp. 360-368.

15. Kauffman, L.H.; Lomonaco, S.J. Braiding Operators are Universal Quantum Gates, New Journal of Physics 2004, Volume 6, 134; Quantum Physics 2004, arXiv preprint quant-ph/04011090v4.

16. Nichita, F.F. Jordan Algebras, Jordan Coalgebras and Unification Theories; Editions Universitaires Europeennes, 2017, ISBN: 978-3-639-62347-5.

17. Nichita, F.F., On The Set-Theoretical Yang-Baxter Equation, Acta Universitatis Apulensis, No. 5 / 2003, 97-100.

18. Nichita, F.F. Introduction to the Yang-Baxter Equation with Open Problems, Axioms 2012, 1(1), 33-37.

19. Marcus, S; Nichita, F.F., On transcendental numbers: new results and a little history, Axioms 2018, $7,15$.

22. Pasarescu, O., On the Classification of Singularities of Normal Gorenstein Surfaces with Ample Anticanonical Divisor, (in Romanian), St. Cerc. Mat., 36(1984), pp. 227-243.

21. Pasarescu, O., The Classification of Singularities of Degenerate del Pezzo Surfaces and Structure Theorems, Preprint IMAR, 1981.

22. Pasarescu, A., Pasarescu, O. Study on Non-standard Convergent Series, An. Univ. Pitesti, 1999.

23. Pasarescu, A., Nonstandard Algebraic Methods in the Study of Analytic Spaces, (in Romanian), Ph.D. Thesis, Geometry Balkan Press, 2003.

24. Popescu, N., Popescu, L., Theory of Categories, Ed. Acad., SijthoffNoordhoff, 1979.

25. Radu, A., Topoi Theory, (in Romanian), Romanian Academy Publishing House, vol I 1981, vol II 1982.

26. Robinson, A. Non-standard Analysis, Princeton University Press, 1974.

27. Rudeanu, S., Boolean function and Equations, North Holland, 1974.

28. Seth, A., 30-Seconds Brain, Ivy Press Limited, 2013.

29. Siegel, J.D. Mind : A Journey to the Heart of Being Human, Mind Your Brain Inc., 2017.

30. Stonier, T. Information and the Internal Structure of the Universe, Springer-Verlag, 1990.

31. Tegmark, M., Our Mathematical Universe: My Quest for the Ultimate Nature of Reality, Knopf, 2014.

32. Walleczek J., Grossing G., Pylkkanen P., Hiley B., eds Emergent Quantum Mechanics, 2019, www.mdpi.com/journal/entropy

33. Zanoci,,C.., Dehghani, M., Tegmark, M., Ensemble Intuition and Excitation in the Human Cortex : an Ising Model Analysis with Uncertainities, arXiv 1810.07253, oct. 2018 